\title{
Genus two partition functions of chiral conformal field theories
}

\author{
Matthias R. Gaberdiel, Christoph A. Keller and \\ Roberto VOLPATO
}

\begin{abstract}
A systematic analysis of the genus two vacuum amplitudes of chiral self-dual conformal field theories is performed. It is explained that the existence of a modular invariant genus two partition function implies infinitely many relations among the structure constants of the theory. All of these relations are shown to be a consequence of the associativity of the operator product expansion, as well as the modular covariance properties of the torus one-point functions. Using these techniques we prove that for the proposed extremal conformal field theories at $c=24 k$ a consistent genus two vacuum amplitude exists for all $k$, but that this does not actually check the consistency of these theories beyond what is already testable at genus one.
\end{abstract}

\section{Introduction}

Usually, a $2 \mathrm{~d}$ conformal field theory is defined by specifying the spectrum of the theory (typically in terms of representations of some chiral algebra), as well as the operator product expansions (OPEs) of the corresponding fields. The consistency conditions require, in particular, that the OPE is associative, and that the theory is modular covariant at genus $g=1$. Given these assumptions (or more precisely, assuming that the polynomial relations of Moore and Seiberg [1] are satisfied) the theory is then also well defined on higher genus Riemann surfaces.

There are, however, situations where a conformal field theory is characterized in a different manner. In particular, in the context of the $\mathrm{AdS}_{3} / \mathrm{CFT}_{2}$ correspondence, the gravity approach does not give access to the algebraic properties of the theory such as its OPEs. Instead, we can only determine the vacuum amplitudes (at arbitrary genus) from the gravity point of view [2-6]. It is an old idea of Friedan and Shenker [7] that a conformal field theory is also uniquely characterized in terms of these partition functions (as functions of their modular parameters). Unlike the usual approach to conformal 
field theory, this avenue has been much less explored. In particular, there are two fundamental questions that are, to our knowledge, still unanswered:

(1) Given the partition functions for all genera, is the conformal field theory defined uniquely?

(2) What are the consistency conditions a family of higher genus partition functions has to satisfy in order to define a consistent conformal field theory?

A little while ago, two of us [8] showed that knowing all genus partition functions determines the current symmetry of the underlying conformal field theory uniquely. Assuming a natural Lie algebraic conjecture, we could also show that these amplitudes fix the representation content with respect to this current algebra up to an overall automorphism of the Lie algebra. While this does not prove (1) - for example, these statements are vacuous for theories that do not have any currents - it gives very strong credence to it.

In this paper we shall begin to address the second question (2). Given the complexity of higher genus amplitudes, we shall only be able to explore the situation at genus $g=2$, and only for chiral (self-dual) theories at $c=24 k$. However, some of the salient features are already visible there. In particular, we shall show that the existence of a modular invariant genus $g=2$ partition function implies infinitely many relations among the structure constants of the underlying conformal field theory; this follows directly from the fact that the vector space of such partition functions is finite dimensional. As we shall prove, all of those relations are a consequence of the associativity of the OPEs (Jacobi identities of the $\mathcal{W}$-algebra), as well as modular covariance at genus $g=1$, in nice agreement with the analysis of Moore and Seiberg. However, we can also identify at least one additional consistency condition (beyond modular invariance) that the vacuum amplitudes have to satisfy in order to define a conformal field theory. This is the condition that the expansion coefficients of the partition functions can actually be written in terms of polynomials of individual structure constants. As we shall show with two examples in Section 5, this is a non-trivial consistency condition which seems to go beyond modular invariance. On the other hand, our analysis also suggests that this is the only additional consistency condition.

One of the main motivations for this work comes from the proposal of Witten [2] regarding extremal conformal field theories. Witten proposed that the dual of pure gravity in $\mathrm{AdS}_{3}$ should be an extremal self-dual chiral conformal field theory with central charge $c=24 k, k \in \mathbb{Z}$, where $k$ is proportional to the AdS radius. Here "extremal" means that the theory contains, apart from the Virasoro descendants of the vacuum, only fields 
with conformal weight $h \geq k+1$. Self-duality implies, in particular, that the character of the vacuum representation must be modular invariant by itself, and these two conditions then fix the vacuum character (and hence the total partition function) completely. For $k=1$ the extremal theory is the famous Monster theory, but the question of whether the theories with $k \geq 2$ exist remains an open problem. Indeed, while the spectrum is modular invariant (by construction), it is far from obvious whether one can define an associative OPE on the corresponding set of fields. Using modular differential equations, two of us $[9,10]$ have argued that the theories should be inconsistent for large $k(k \geq 42)$, but unfortunately there is still a small loophole in the argument.

For the extremal ansatz at $k=2$ and $k=3$, it was shown in [2] (for $k=2$ ) and [4] that one can define a consistent genus $g=2$ partition function. Given that the existence of a modular invariant genus $g=2$ vacuum amplitude implies infinitely many relations among the structure constants of the theory (see above), this would appear to represent a highly non-trivial consistency check for these theories. However, as we shall also explain in this paper, this is somewhat misleading. Indeed, many of the relations involve in fact coefficients that are not otherwise known (and thus do not lead to any real "constraints"), while the remaining "testable" relations (of which there are still infinitely many!) turn out to be a consequence of the associativity of the Virasoro algebra and the modular covariance of certain simple 1-point functions at genus $g=1$. In fact, we can prove rather generally (see Theorem 4.1 in Section 4) that a "consistent" genus $g=2$ partition function exists for a large class of putative theories, that include, in particular, the extremal ansatz at arbitrary $k$. Furthermore, it is clear from the assumptions of this theorem that the existence of a genus $g=2$ amplitude does not impose any constraints beyond those that can already be analysed at genus one.

The paper is organized as follows. In Section 2 we explain that the space of modular invariant genus two vacuum amplitudes is finite dimensional for fixed $c=24 k$. We also identify different sets of expansion coefficients that determine the genus two partition function uniquely. In Section 3 the various different expansion coefficients are interpreted from a conformal field theory point of view. We identify the linear relations between the different expansion coefficients that arise as a consequence of modular invariance at genus two, and show that they are a consequence of the associativity of the OPE and modular covariance at genus one. Some explicit examples are worked out in Section 3.3. In Section 4 we apply these techniques to prove that a consistent genus two vacuum amplitude exists for the extremal ansatz at arbitrary $k$. We also show that this property is quite generic, and that it does not actually test any consistency conditions beyond what is already 
testable at genus one - see Theorem 4.1. Finally, in Section 5 we identify the additional consistency condition that has to be satisfied in order for the vacuum amplitudes to define a consistent conformal field theory. We also estimate the behaviour of this constraint at large genus, and suggest that it will eventually (i.e., for sufficiently large genus) become very constraining for the extremal ansatz. Section 6 contains our conclusions, and there are a number of appendices where some of the more technical material has been collected.

\section{Genus two modular forms}

As was, for example, explained in [8], the genus two partition function of a meromorphic conformal field theory at central charge $c=24 k$ is of the form

$$
Z_{2}=\frac{W}{F^{12 k}},
$$

where $W$ is a modular form of weight $12 k$, while $F^{12 k}$ serves as a reference partition function - it describes the chiral contribution of $24 k$ uncompactified free bosons to the genus two partition function. At genus $g=2$ the modular form $W$ may be taken to be a Siegel modular form $W(\Omega)$, where $\Omega$ is the Riemann period matrix of the corresponding Riemann surface. The period matrices provide a parametrization of the moduli space of Riemann surfaces with respect to which modular transformations assume a particularly simple form; for a more detailed explanation of all of these see Appendices A and B.

In order to analyse the factorization properties of partition functions under degeneration limits, however, other parametrizations are more appropriate. The most basic one, which we will call the "sewn-tori coordinates", comes from the so-called plumbing fixture construction where one joins two tori (of modular parameters $q_{1}$ and $q_{2}$ ) by a cylinder whose modular parameter is described by a third variable $\epsilon$, see figure 1 . This is the parametrization that was used in the work of Mason and Tuite [11-13].

Alternatively, we may use that any modular form $W(\Omega)$ may be lifted to an automorphic form $\hat{W}$ on the Schottky space $\mathfrak{S}_{2}$ (that forms a finite covering of the moduli space of genus two surfaces, see Appendix A.3). The Schottky space $\mathfrak{S}_{2}$ can be identified with the open subset of $\mathbb{C}^{3}$ defined by

$$
\mathfrak{S}_{2}:=\left\{\left(p_{1}, p_{2}, x\right) \in \mathbb{C}^{3}|x \neq 0,1,0<| p_{i} \mid<\min \{|x|, 1 /|x|\}, i=1,2\right\},
$$



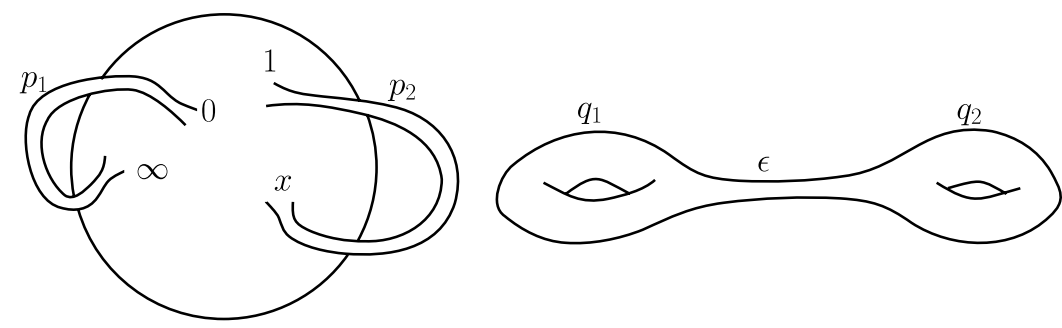

Figure 1: To the left, the geometric interpretation of the Schottky coordinates $p_{1}$ and $p_{2}$; the third coordinate $x$ is given by the cross-section of the insertion points. To the right, the geometric interpretation of the sewn tori coordinates $q_{1}, q_{2}, \epsilon$.

where the relation to the usual Riemann period matrix $\Omega$ is

$e^{2 \pi i \Omega_{11}}=p_{1}\left(1+\mathcal{O}\left(p_{2}\right)\right), \quad e^{2 \pi i \Omega_{22}}=p_{2}\left(1+\mathcal{O}\left(p_{1}\right)\right), \quad e^{2 \pi i \Omega_{12}}=x+\mathcal{O}\left(p_{1} p_{2}\right)$.

This parametrization is appropriate to describe the degeneration where the genus two surface becomes a sphere with two thin handles connecting 0 and $\infty$, and $x$ and 1 , respectively, see figure 1 and Appendix A. The Schottky parametrization has also been applied to the analysis of higher loop string amplitudes, see for example [14-19].

In the following we shall analyse the structure of $Z_{2}$ as in (2.1), using just modularity and regularity properties. In particular, we shall take $W$ to be any Siegel modular form of weight $12 k$ (and $\hat{W}$ its lift to the Schottky space), and we shall assume that $Z_{2}$ has smooth limits at the boundary of moduli space. However, we shall not assume that the function $W$ (or $Z_{2}$ ) arises from a consistent conformal field theory.

\subsection{Siegel modular forms of degree $g=2$}

At genus two, the space of Siegel modular forms of degree $g=2$ and even weight is freely generated by

$$
\psi_{4}, \quad \psi_{6}, \quad \chi_{10}, \quad \chi_{12},
$$

where the subscript denotes the modular weight (see Appendix A.2). Since we are only interested in forms of weight $12 k$, it is useful to introduce a set of generators for the corresponding subring

$$
\psi_{4}^{3}, \quad \psi_{12}, \quad \chi_{12}, \quad \chi_{12 w_{d}}^{(d)} .
$$


300 Matthias R. Gaberdiel, Christoph A. Keller and Roberto Volpato

Here we have defined $\psi_{12}=\frac{\psi_{4}^{3}-\psi_{6}^{2}}{1728}$, and $\chi_{12 w_{d}}^{(d)}$ is the modular form of smallest weight $w=12 w_{d}$ with $w_{d} \in \mathbb{N}$, that contains as a factor $\chi_{10}^{d}$. More explicitly, we have $\chi_{0}^{(0)}=1$ as well as

$$
\begin{array}{ll}
\chi_{24}^{(1)}=\chi_{10} \psi_{4}^{2} \psi_{6}, & \chi_{24}^{(2)}=\chi_{10}^{2} \psi_{4}, \\
\chi_{36}^{(3)}=\chi_{10}^{3} \psi_{6}, & \chi_{48}^{(4)}=\chi_{10}^{4} \psi_{4}^{2}, \\
\chi_{60}^{(5)}=\chi_{10}^{5} \psi_{4} \psi_{6}, & \chi_{60}^{(6)}=\chi_{10}^{6},
\end{array}
$$

and for arbitrary $d>6$ we define recursively

$$
\chi_{12 w_{d}}^{(d)}=\chi_{10}^{6} \chi_{12\left(w_{d}-5\right)}^{(d-6)}, \quad d>6 .
$$

Note that $w_{d}$ is given by the formula

$$
w_{d}=d-\left\lfloor\frac{d}{6}\right\rfloor+\delta_{1, d \bmod 6} .
$$

The space of modular forms of $w=12 k$ is then spanned by the modular forms

$$
\phi_{a, b, c, d}=\psi_{4}^{3 a} \psi_{12}^{b} \chi_{12}^{c} \chi_{12 w_{d}}^{(d)},
$$

where $a, b, c, d$ are non-negative integers in the set

$$
\mathcal{P}_{k}=\left\{(a, b, c, d): a+b+c+w_{d}=k\right\} .
$$

The function $Z_{2}$ in (2.1) thus takes the form

$$
\begin{aligned}
Z_{2} & =\sum_{(a, b, c, d) \in \mathcal{P}_{k}} f_{a b c d} \frac{\hat{\phi}_{a, b, c, d}}{F^{12 k}} \\
& =\sum_{(a, b, c, d) \in \mathcal{P}_{k}} f_{a b c d}\left(\frac{\hat{\psi}_{4}^{3}}{F^{12}}\right)^{a}\left(\frac{\hat{\psi}_{12}}{F^{12}}\right)^{b}\left(\frac{\hat{\chi}_{12}}{F^{12}}\right)^{c} \frac{\hat{\chi}_{12 w_{d}}^{(d)}}{F^{12 w_{d}}}
\end{aligned}
$$


where $f_{a b c d}$ are some constants, and $\hat{\psi}$ and $\hat{\chi}$ are the lifts of $\psi$ and $\chi$, respectively, to the Schottky space. The various factors have an expansion as

$$
\begin{gathered}
\frac{\hat{\psi}_{4}^{3}}{F^{12}}=1+744\left(p_{1}+p_{2}\right)+\cdots, \\
\frac{\hat{\psi}_{12}}{F^{12}}=p_{1}+p_{2}+\cdots, \\
\frac{\hat{\chi}_{12}}{F^{12}}=p_{1} p_{2}\left(1+\frac{1}{12} \frac{(x-1)^{2}}{x}\right)+\cdots, \\
\frac{\hat{\chi}_{12 w_{d}}^{(d)}}{F^{12 w_{d}}}=\frac{(x-1)^{2 d}}{x^{d}}\left(-\frac{1}{4} p_{1} p_{2}+\cdots\right)^{d},
\end{gathered}
$$

where the ellipses denote higher powers in $p_{1}$ or $p_{2}$. The contribution of the term proportional to $f_{a b c d}$ is thus

$$
\frac{\hat{\phi}_{a, b, c, d}}{F^{12 k}}=\left(p_{1}^{b+c+d} p_{2}^{c+d}+p_{1}^{c+d} p_{2}^{b+c+d}\right)\left(\left(-\frac{1}{4}\right)^{d} f(x)+\cdots\right),
$$

where $f(x)$ is explicitly given as

$$
f(x)=\frac{(x-1)^{2 d}}{x^{d}} \sum_{l=0}^{c}\left(\begin{array}{l}
c \\
l
\end{array}\right)\left(\frac{1}{12}\right)^{l} \frac{(x-1)^{2 l}}{x^{l}} .
$$

\subsection{Expansion coefficients}

The above argument shows that the space of $g=2$ modular forms of weight $12 k$ is finite dimensional; indeed, the set $\mathcal{P}_{k}$ has

$$
\left|\mathcal{P}_{k}\right|=\frac{k^{3}}{5}+\mathcal{O}\left(k^{2}\right)
$$

elements. In particular, this implies that the genus two partition function $Z_{2}$ must be uniquely determined in terms of a finite set of coefficients in some suitable coordinate expansion. As we have mentioned above, there are two different classes of coordinates that one may naturally use.

2.2.1. Schottky expansion. In the Schottky parametrization the lift of $Z_{2}$ to the Schottky space has the power series expansion

$$
\hat{Z}_{2}=\frac{\hat{W}}{F^{12 k}}=\sum_{h_{2}, h_{1}=0}^{\infty} C_{h_{1}, h_{2}}(x) p_{1}^{h_{1}} p_{2}^{h_{2}},
$$


where $C_{h_{1}, h_{2}}(x)$ is a rational function of $x$ whose only poles are at $0,1, \infty$; the order of the poles are bounded by

$C_{h_{1}, h_{2}}(x) \stackrel{x \rightarrow 0}{\sim} \mathcal{O}\left(x^{-h_{1}-h_{2}}\right), \quad C_{h_{1}, h_{2}}(x) \stackrel{x \rightarrow 1}{\sim} \mathcal{O}(1), \quad C_{h_{1}, h_{2}}(x) \stackrel{x \rightarrow \infty}{\sim} \mathcal{O}\left(x^{h_{1}+h_{2}}\right)$.

For example, the first of these properties can be proven by restricting $\hat{Z}_{2}$ to the curve $\left(p_{1}(t), p_{2}(t), x(t)\right) \subset \mathfrak{S}_{2}$, with

$$
x(t)=t, \quad p_{1}(t)=t / 2, \quad p_{2}(t)=t / 2,
$$

where $t \in \mathbb{C}$ and $0<|t|<1 / 2$. The requirement that $\hat{Z}_{2}$ has a finite limit as $|t| \rightarrow 0$ (i.e., at the boundary of moduli space) then leads to the first bound in (2.20); the other bounds can be proven similarly.

By construction $\hat{Z}_{2}$ is also modular invariant, and this implies that the functions $C_{h_{1}, h_{2}}(x)$ must satisfy

$$
\begin{aligned}
& C_{h_{1}, h_{2}}(x)=C_{h_{2}, h_{1}}(x), \\
& C_{h_{1}, h_{2}}(x)=C_{h_{1}, h_{2}}(1 / x) .
\end{aligned}
$$

Indeed, the first identity comes from considering the modular transformation that acts on the usual fundamental cycles $\left\{\alpha_{1}, \alpha_{2}, \beta_{1}, \beta_{2}\right\}$ as $\left(\alpha_{1}, \alpha_{2}, \beta_{1}\right.$, $\left.\beta_{2}\right) \mapsto\left(\alpha_{2}, \alpha_{1}, \beta_{2}, \beta_{1}\right)$, while the second one comes from the transformation $\left(\alpha_{1}, \alpha_{2}, \beta_{1}, \beta_{2}\right) \mapsto\left(\alpha_{1}, \alpha_{2}^{-1}, \beta_{1}, \beta_{2}^{-1}\right)$. Because of the first equation in (2.22) we may, from now on, consider only the functions $C_{h_{1}, h_{2}}(x)$ with $h_{2} \leq h_{1}$.

For the following it will be useful to expand $C_{h_{1}, h_{2}}(x)$ in a power series. A particularly simple expansion is

$$
C_{h_{1}, h_{2}}(x)=\sum_{l=0}^{h_{1}+h_{2}} C_{h_{1}, h_{2} ; l}^{(*)} \frac{(x-1)^{2 l}}{x^{l}}
$$

since $(2.20)$ and $(2.22 \mathrm{~b})$ imply that the sum on the right-hand side is finite. Since $W$ is a modular form of weight $12 k$, it is clear that these coefficients can be expressed in terms of linear combinations of the $f_{a b c d}$ that appear in (2.11), i.e.,

$$
C_{h_{1}, h_{2} ; l}^{(*)}=\sum_{(a, b, c, d) \in \mathcal{P}_{k}} M_{\left(h_{1}, h_{2} ; l\right)}^{a b c d} f_{a b c d}
$$

where $M$ is a matrix that depends on $k$. We should furthermore expect that we can invert this relation. However, since there are infinitely many 
coefficients of the form $C_{h_{1}, h_{2} ; l}^{(*)}$ - for each fixed $h_{1}$ and $h_{2}, l$ only takes the finitely many values $l=0, \ldots, h_{1}+h_{2}$, but there are infinitely many values for $h_{1}$ and $h_{2}$ - we need to understand more precisely which of these coefficients are in fact independent. By comparing (2.23) with (2.16) it is easy to see that we may take the independent coefficients to be labelled by

$$
\mathcal{P}_{k}^{(*)}=\left\{\left(h_{1}=b+c+d, h_{2}=c+d ; l=d\right): b, c, d \in \mathbb{N}, b+c+w_{d} \leq k\right\} .
$$

In particular, this then implies that we can express the $f_{a b c d}$ as

$$
f_{a b c d}=\sum_{\left(h_{1}, h_{2} ; l\right) \in \mathcal{P}_{k}^{(*)}} \hat{M}_{a b c d}^{\left(h_{1}, h_{2} ; l\right)} C_{h_{1}, h_{2} ; l^{*}}^{(*)}
$$

Note that the set $\mathcal{P}_{k}^{(*)}$ consists of all triplets $\left(h_{1}, h_{2}, l\right)$ of integers for which

$$
0 \leq l \leq h_{2} \leq h_{1} \leq k+l-w_{l}, \quad h_{1}, h_{2}, l \in \mathbb{Z}
$$

Obviously the inequalities (2.27) only have a solution provided that $w_{l} \leq k$. Because of (2.8), we have the bounds

$$
\frac{5}{6} l \leq w_{l} \leq l+1
$$

and thus a necessary condition for $\left(h_{1}, h_{2}, l\right) \in \mathcal{P}_{k}^{(*)}$ is

$$
\left(h_{1}, h_{2}, l\right) \in \mathcal{P}_{k}^{(*)} \Longrightarrow 0 \leq l \leq h_{2} \leq h_{1} \leq k+\frac{l}{6} \leq \frac{6}{5} k
$$

where in the last inequality we have used that $l \leq k+\frac{l}{6}$ implies $l \leq \frac{6}{5} k$. On the other hand, using the upper bound from (2.28) implies that a sufficient condition for $\left(h_{1}, h_{2}, l\right)$ to be in $\mathcal{P}_{k}^{(*)}$ is

$$
0 \leq l \leq h_{2} \leq h_{1} \leq k-1 \Longrightarrow\left(h_{1}, h_{2}, l\right) \in \mathcal{P}_{k}^{(*)} .
$$

The structure of $\mathcal{P}_{k}^{(*)}$ is sketched in figure 2 . 
304 Matthias R. Gaberdiel, Christoph A. Keller and Roberto Volpato

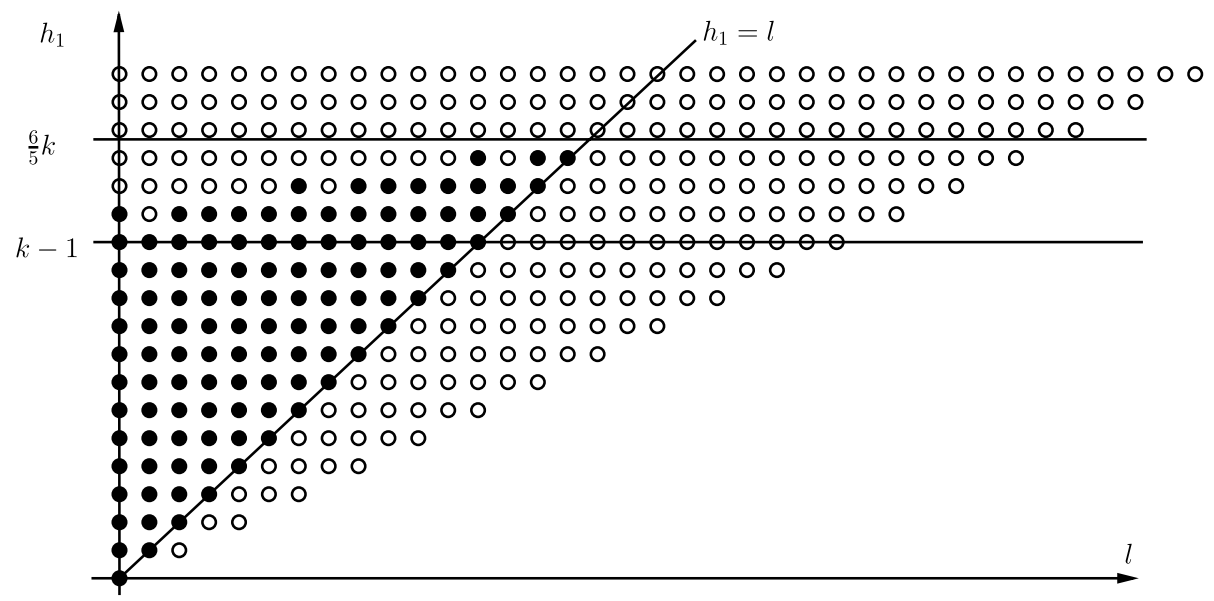

Figure 2: A graphical representation of the set $\mathcal{P}_{k}^{(*)}$ for $k=13$. Each (either white or black) circle in the diagram denotes a pair $\left(h_{1}, l\right)$ for which we can find a $h_{2} \leq h_{1}$ with $0 \leq l \leq h_{1}+h_{2}$. Black circles denote pairs $\left(h_{1}, l\right)$ for which at least one such choice of $h_{2}$ corresponds to an element in $\mathcal{P}_{k}^{(*)}$.

2.2.2. Expansion in sewn tori coordinates. Later on we shall also need the description of the genus two partition function in terms of the sewn tori coordinates $q_{1}, q_{2}$ and $\epsilon$, see figure 1 . With respect to these coordinates we have an expansion as

$$
Z_{2}=\sum_{h_{1}, h_{2}, l=0}^{\infty} \mathcal{D}_{h_{1}, h_{2} ; l} q_{1}^{h_{1}} q_{2}^{h_{2}} \epsilon^{2 l}
$$

The coefficients $\mathcal{D}_{h_{1}, h_{2} ; l}$ are symmetric under the exchange of $h_{1} \leftrightarrow h_{2}$, and we may therefore restrict ourselves to considering the terms with $h_{1} \geq h_{2}$. The explicit relation between the sewn tori and the Schottky coordinates is given by

$$
\begin{gathered}
q_{1}=p_{1}\left(1+\mathcal{O}\left(p_{1}, p_{2}\right)\right), \quad q_{2}=p_{2}\left(1+\mathcal{O}\left(p_{1}, p_{2}\right)\right), \\
\epsilon=(x-1)\left(1+\mathcal{O}(x-1)^{2}+\mathcal{O}\left(p_{1}, p_{2}\right)\right) .
\end{gathered}
$$

Obviously, again only finitely many of these coefficients are independent, and we may take them to be $\mathcal{D}_{h_{1}, h_{2} ; l}$, with $\left(h_{1}, h_{2} ; l\right)$ in

$$
\mathcal{P}_{k}^{(\mathcal{D})}=\left\{\left(h_{1}, h_{2} ; l\right): 0 \leq l \leq h_{2} \leq h_{1} \leq k+l-w_{l}\right\}
$$




\section{The conformal field theory perspective}

Up to now we have analysed the modular properties of the partition functions $Z_{2}$, but we have not assumed that they arise from an underlying conformal field theory. As we have explained in the previous section, there are infinitely many relations between the expansion coefficients of $C_{h_{1}, h_{2}}(x)$ and the coefficients $\mathcal{D}_{h_{1}, h_{2} ; l}$ (since all of them are determined in terms of the finitely many coefficients labelled by $\mathcal{P}_{k}$ ). These relations encode constraints the underlying conformal field theory has to satisfy in order to define a consistent genus two partition function. In the following, we want to exhibit these constraints more explicitly.

\subsection{Invariants of the conformal field theory}

To start with we need to explain the conformal field theory interpretation of the different expansion coefficients.

3.1.1. The Schottky expansion. In the Schottky parametrization it is clear from the geometrical definition (see Appendix C) that the coefficient functions $C_{h_{1}, h_{2}}(x)$ have the interpretation

$$
\begin{array}{r}
C_{h_{1}, h_{2}}(x)=\sum_{\substack{\phi_{1}, \psi_{1} \in \mathcal{H}_{h_{1}} \\
\phi_{2}, \psi_{2} \in \mathcal{H}_{h_{2}}}} G_{\phi_{1} \psi_{1}}^{-1} G_{\phi_{2} \psi_{2}}^{-1}\left\langle V^{\text {out }}\left(\phi_{1}, \infty\right)\right. \\
\left.V^{\text {out }}\left(\phi_{2}, x\right) V^{\text {in }}\left(\psi_{2}, 1\right) V^{\text {in }}\left(\psi_{1}, 0\right)\right\rangle .
\end{array}
$$

Here $G_{\phi \psi}$ is the metric on the space of states (with $G_{\phi \psi}^{-1}$ the inverse metric), and the sums over $\phi_{j}, \psi_{j}$ run over a basis of states at conformal weight $h_{j}$. Finally, $V^{\text {in }}$ and $V^{\text {out }}$ are defined as in (C.25) and (C.26), and the 4-point correlator is evaluated on the sphere. Note that the crossing symmetry of these correlation functions implies directly (2.22), see Equations (C.32) and (C.33). Furthermore, the regularity conditions (2.20) are a consequence of the property of the $L_{0}$-spectrum of the conformal field theory to be bounded from below by zero.

It is convenient to restrict the sum over the states at conformal dimension $h_{2}$ to the quasiprimary states $\mathcal{H}_{h_{2}}^{q p}$, leading to the "quasiprimary functions"

$$
\mathcal{C}_{h_{1}, h_{2}}(x)=(x-1)^{2 h_{2}} \sum_{\phi, \psi \in \mathcal{H}_{h_{2}}^{q p}} G_{\phi \psi}^{-1} \operatorname{Tr}_{\mathcal{H}_{h_{1}}}(V(\psi, 1) V(\phi, x)) .
$$


Because the contribution of the $L_{-1}$-descendant states can be described in terms of differential operators, the general case is then of the form

$$
C_{h_{1}, h_{2}}(x)=\sum_{h_{2}^{\prime} \leq h_{2}} K_{h_{2}, h_{2}^{\prime}}(x) \mathcal{C}_{h_{1}, h_{2}^{\prime}}(x),
$$

where $K_{h_{2}, h_{2}^{\prime}}$ are some differential operators (that are independent of the theory in question). Any linear relations between the coefficient functions $C_{h_{1}, h_{2}}(x)$ must therefore come from linear relations between the quasiprimary functions $\mathcal{C}_{h_{1}, h_{2}}(x)$. In the following we shall therefore restrict our attention to these quasiprimary correlation functions. Since $\mathcal{C}_{h_{1}, h_{2}}$ still satisfy $(2.22 \mathrm{~b})$ and $(2.20)$, we can expand them as in $(2.23)$

$$
\mathcal{C}_{h_{1}, h_{2}}(x)=\sum_{l=0}^{h_{1}+h_{2}} \mathcal{C}_{h_{1}, h_{2} ; l}^{(*)} \frac{(x-1)^{2 l}}{x^{l}} .
$$

However, from the point of view of conformal field theory, the expansion,

$$
\mathcal{C}_{h_{1}, h_{2}}(x)=\sum_{l=0}^{\infty} \mathcal{C}_{h_{1}, h_{2} ; l}^{(0)}(x-1)^{2 h_{2}} x^{l-h_{1}-h_{2}}
$$

which converges for $|x|<1$, is more natural. Indeed, while the conformal field theory interpretation of the coefficients $\mathcal{C}_{h_{1}, h_{2} ; l}^{(*)}$ is not immediate, Equation (3.2) implies that

$$
\mathcal{C}_{h_{1}, h_{2} ; l}^{(0)}=\sum_{\phi, \psi \in \mathcal{H}_{h_{2}}^{q p}} G_{\phi \psi}^{-1} \operatorname{Tr}_{\mathcal{H}_{h_{1}}}\left(V_{-h_{1}+l}(\psi) V_{h_{1}-l}(\phi)\right) .
$$

It is clear that we can express the $\mathcal{C}_{h_{1}, h_{2} ; l}^{(0)}$ coefficients in terms of the $\mathcal{C}_{h_{1}, h_{2} ; l}^{(*)}$ coefficients, and vice versa. Indeed, we can rewrite (3.4) for any $L \geq 0$ as

$$
\begin{aligned}
\mathcal{C}_{h_{1}, h_{2}}(x) & =\sum_{l=0}^{L} \mathcal{C}_{h_{1}, h_{2} ; h_{1}+h_{2}-l}^{(*)}(x-1)^{2 h_{2}} \frac{(x-1)^{2 h_{1}-2 l}}{x^{h_{1}+h_{2}-l}}+\mathcal{O}\left(x^{L-h_{1}-h_{2}+1}\right) \\
& =\sum_{l=0}^{L} \mathcal{C}_{h_{1}, h_{2} ; l}^{(0)}(x-1)^{2 h_{2}} x^{l-h_{1}-h_{2}}+\mathcal{O}\left(x^{L-h_{1}-h_{2}+1}\right),
\end{aligned}
$$

from which we conclude that we can express the coefficients

$$
\begin{aligned}
& \left\{\mathcal{C}_{h_{1}, h_{2} ; l}^{(0)}: l=0, \ldots, L\right\} \quad \text { as linear combinations of } \\
& \quad\left\{\mathcal{C}_{h_{1}, h_{2} ; h_{1}+h_{2}-l}^{(*)}: l=0, \ldots, L\right\}
\end{aligned}
$$


and vice versa. In particular, given the definition of $\mathcal{P}_{k}^{(*)}$, this implies that the independent $\mathcal{C}_{h_{1}, h_{2} ; l}^{(0)}$ coefficients can be taken to be labelled by

$$
\mathcal{P}_{k}^{(0)}=\left\{\left(h_{1}, h_{2} ; h_{1}+h_{2}-l\right): 0 \leq l \leq h_{2} \leq h_{1} \leq k+l-w_{l}\right\} .
$$

The fact that only the invariants with $l=0, \ldots, h_{1}+h_{2}$ can be independent is actually directly a consequence of the crossing symmetry $(2.22 \mathrm{~b})$. Equation (3.8) holds for a general $L$ if we define $\mathcal{C}_{h_{1}, h_{2} ; l}^{(*)}=0$ for $l<0$ or $l>h_{1}+h_{2}$.

3.1.2. The sewn tori coordinate expansion. The conformal field theory interpretation of the coefficients $\mathcal{D}_{h_{1}, h_{2} ; l}$ in $(2.31)$ is more immediate. In fact, it follows directly from the geometric interpretation of the sewn tori coordinates (see figure 1) that the expansion coefficients have the interpretation

$$
\mathcal{D}_{h_{1}, h_{2} ; l}=\sum_{\phi, \psi \in \mathcal{H}_{[2 l]}} G_{\phi \psi}^{-1} \operatorname{Tr}_{\mathcal{H}_{h_{1}}}\left(V_{0}(\phi)\right) \operatorname{Tr}_{\mathcal{H}_{h_{2}}}\left(V_{0}(\psi)\right)
$$

This was taken to be the starting point in the analysis of Mason and Tuite $[11,12]$. Here $\mathcal{H}_{[h]}$ denotes the eigenspace (with eigenvalue $h$ ) of the operator $L_{[0]}$, the scaling operator on the torus, defined by

$$
L_{[0]}=L_{0}+\sum_{n=1}^{\infty} \frac{(-1)^{n+1}}{n(n+1)} L_{n}
$$

Physically, this modified scaling operator comes from the conformal transformation to torus coordinates. Mathematically, its justification is that the torus one point function $Z_{\phi}$ defined by

$$
Z_{\phi}(q)=\operatorname{Tr}_{\mathcal{H}}\left(V_{0}(\phi) q^{L_{0}-k}\right), \quad q=e^{2 \pi i \tau},
$$

with $\tau$ the modular parameter on the torus, is an elliptic modular function of weight $h$, provided that $\phi$ has $L_{[0]}$ eigenvalue $h[20]$. Note that $L_{0}=L_{[0]}$ on Virasoro primary states.

This concludes our discussion of the conformal field interpretation of the expansion coefficients. For a consistent conformal field theory one also usually requires that the theory has a unique $\mathrm{SL}(2, \mathbb{C})$-invariant vacuum. This condition puts some additional constraints on the above parameters. 
In particular, it implies that

$$
\mathcal{D}_{h_{1}, h_{2} ; 0}=N_{h_{1}} N_{h_{2}},
$$

where $N_{i}=\operatorname{dim} \mathcal{H}_{i}$. As we have seen in Section 2, the independent coefficients $\mathcal{D}_{h_{1}, h_{2} ; l}$ are labelled by $\mathcal{P}_{k}^{(\mathcal{D})}$ defined in $(2.33)$. For $l=0$ the independent coefficients are thus characterized by $0 \leq h_{2} \leq h_{1} \leq k$. Because of (3.13) this implies that the dimensions $N_{i}$ for $i \leq k$ are free parameters, while the dimensions $N_{j}$ with $j>k$ cannot be independently chosen. This fits in perfectly with the constraints obtained by imposing modular invariance of the genus $g=1$ torus partition function $[2,21]$ (see also the discussion in the following section). In fact, the latter point of view also implies that the invariants of the form $\mathcal{D}_{h_{1}, h_{2} ; 0}$ with $h_{1}>k$ depend only on the $\mathcal{D}_{h_{1}, h_{2} ; 0}$ with $h_{2} \leq h_{1} \leq k$.

\subsection{Interpretation of the linear relations}

As we have seen in Section 2, consistency of the conformal field theory at genus $g=2$ implies that the invariants (3.6) and (3.10) must satisfy a large number of linear relations. On the other hand, the analysis of Moore and Seiberg [1] shows that the consistency of a conformal field theory at higher genus is a consequence of the associativity of the OPE on the sphere, as well as the modular covariance of the torus one-point functions. Indeed, for the self-dual theories we are discussing there, there is only one irreducible representation, namely the vacuum representation itself, and the fusing and braiding matrices are trivial. Thus, the polynomial relations of [1] are obviously satisfied provided that the chiral algebra is itself consistent, i.e., that it has an associative OPE. In addition, one has to require that the one-point functions on the torus are modular covariant of appropriate weight (so that the associated $S$ matrix is indeed unity). Under these conditions the analysis of [1] implies consistency at arbitrary genus. Thus, the linear relations of the invariants (3.6) and (3.10) must be a consequence of (1) associativity of the OPE and (2) modular covariance at genus one.

In order to explain that this is indeed so, we shall proceed as follows. We shall first identify (see Section 3.2.1) which relations follow from the associativity of the OPE on the sphere, and which are a consequence of the modular covariance of the torus one point functions (see Section 3.2.2). Then we shall show (see Section 3.2.3) that taking these relations together allows us to relate any invariant of the theory to a linear combination of invariants associated to $\mathcal{P}_{k}^{(*)}$, thus mirroring the relations that arise from 
modular invariance at genus $g=2$. To prove that the two sets of relations are actually the same, we note that the theta series of even self-dual lattices span the space of modular forms of degree $g=2$ and weight $w=0 \bmod 4$ $[22,23]$. The corresponding lattice conformal field theories are consistent conformal field theories at arbitrary genus, and thus must satisfy both sets of constraints. Since the constraints take the form of a system of linear equations, it follows that the two sets are in fact equivalent.

3.2.1. Relations from the associativity of the OPE. Let us begin with collecting the relations that follow from the associativity of the OPE on the sphere. From the point of view of conformal field theory the expansions (3.4) and (3.5) are different expansions of a certain four-point function on the sphere, see Equation (3.2). The consistency of the chiral algebra implies, in particular, that these functions are well-defined, irrespective of how one expands them. In turn, this means that associativity of the OPE implies the relations (3.8). By a similar argument one can also show, assuming the associativity of the OPE, that we can express the invariants (3.10) in terms of the invariants appearing in (3.4), i.e., that

$$
\begin{aligned}
& \left\{\mathcal{C}_{h_{1}, h_{2}^{\prime} ; l}^{(*)}: l=0, \ldots, L, \quad h_{2}^{\prime} \leq h_{2}\right\} \text { are linear comb. of } \\
& \quad\left\{\mathcal{D}_{h_{1}, h_{2}^{\prime} ; l}: l=0, \ldots, L, \quad h_{2}^{\prime} \leq h_{2}\right\}
\end{aligned}
$$

and vice versa; since this is slightly technical, the proof is given in Appendix D.1. The associativity of the OPE finally implies that the traces appearing in (3.6) are cyclic. In particular, we therefore have the identity

$$
\begin{aligned}
\mathcal{C}_{h_{1}, h_{2} ; l}^{(0)} & =\sum_{\phi, \psi \in \mathcal{H}_{h_{2}}^{q p}} G_{\phi \psi}^{-1} \operatorname{Tr}_{\mathcal{H}_{h_{1}}}\left(V_{-h_{1}+l}(\phi) V_{h_{1}-l}(\psi)\right) \\
& =\sum_{\phi, \psi \in \mathcal{H}_{h_{2}}^{q p}} G_{\phi \psi}^{-1} \operatorname{Tr}_{\mathcal{H}_{l}}\left(V_{h_{1}-l}(\psi) V_{-h_{1}+l}(\phi)\right)=\mathcal{C}_{l, h_{2} ; h_{1}}^{(0)} .
\end{aligned}
$$

Altogether we therefore see that associativity of the OPE implies the relations (3.8), (3.14) and (3.15).

\subsubsection{Relations from modular covariance of the torus one-point} function. Next we turn to the relations that follow from the modular covariance of the torus one-point functions. As was mentioned before in Section 3.1.2, for each $\phi \in \mathcal{H}_{[h]}$, i.e., each $\phi \in \mathcal{H}$ with $L_{[0]} \phi=h \phi$, the onepoint function on the torus $Z_{\phi}$ has modular weight $h$ and is holomorphic for $\tau$ in the upper half plane, except for a pole of order at most $k$ at $q=0$ [20]. 
We denote the space of such functions by $M_{h, k}$. It is not difficult to show that $M_{h, k}$ is isomorphic to the space $M_{12 k+h, 0}$ of (holomorphic) modular forms of weight $12 k+h$ (without any poles): indeed, given an element in $M_{h, k}$, the corresponding modular form is obtained by multiplication with $\Delta^{k}$, where $\Delta$ is the unique modular form of weight 12 and leading term $\Delta(q) \sim q+\mathcal{O}\left(q^{2}\right)$.

The ring of modular forms is freely generated by the Eisenstein series $G_{4}$ and $G_{6}$, and thus there are only modular forms of even weight. The dimension of $M_{h, k}$, for even $h$, equals

$$
d_{h, k}=\operatorname{dim}\left(M_{h, k}\right)=\operatorname{dim}\left(M_{12 k+h}\right)=k+\left\lfloor\frac{h}{12}\right\rfloor+1-\delta_{2, h \bmod 12} .
$$

For $h=2 l$ this can be rewritten, using the definition of $w_{l}$ in $(2.8)$, as

$$
d_{2 l, k}=k+l-w_{l}+1
$$

It is convenient to choose a basis $\xi_{l}^{h, k}(q), l=0, \ldots, d_{h, k}-1$, of $M_{h, k}$ such that

$$
\xi_{l}^{h, k}(q)=q^{-k}\left(q^{l}+\mathcal{O}\left(q^{d_{h, k}}\right)\right)
$$

Then for any $\phi \in \mathcal{H}_{[h]}$, we can write the torus one-point function as

$$
Z_{\phi}(q)=\sum_{n=0}^{\infty} q^{n-k} \operatorname{Tr}_{\mathcal{H}_{n}}\left(V_{0}(\phi)\right)=\sum_{l=0}^{d_{h, k}-1} \xi_{l}^{h, k}(q) \operatorname{Tr}_{\mathcal{H}_{l}}\left(V_{0}(\phi)\right)
$$

In particular, this therefore implies that the first $d_{h, k}$ coefficients of the $q$-expansion of (3.19) determine all remaining power series coefficients.

Given the definition of the invariants $\mathcal{D}_{h_{1}, h_{2} ; l}(3.10)$, and by the obvious identity $\mathcal{D}_{h_{1}, h_{2} ; l}=\mathcal{D}_{h_{2}, h_{1} ; l}$, it is therefore clear that the invariants with $0 \leq h_{2} \leq h_{1} \leq d_{2 l, k}-1$ determine all other $\mathcal{D}_{h_{1}, h_{2} ; l}$ invariants. Since $d_{2 l, k}-$ $1=k+l-w_{l}$ this means that we can always express

$$
\mathcal{D}_{h_{1}, h_{2} ; l} \quad \text { as a linear combination of } \quad\left\{\mathcal{D}_{h_{1}^{\prime}, h_{2}^{\prime} ; l}: h_{2}^{\prime} \leq h_{1}^{\prime} \leq k+l-w_{l}\right\} \text {. }
$$

3.2.3. Completeness of these relations. Finally, it remains to show that the conformal field theory relations explained in Sections 3.2.1 and 3.2.2 allow us to relate any invariant in terms of the invariants $\mathcal{C}_{h_{1}, h_{2} ; l}^{(*)}$ with $\left(h_{1}, h_{2} ; l\right) \in \mathcal{P}_{k}^{(*)}$. The precise statement is given by the following theorem. 
Theorem 3.1. The relations (3.8), (3.14), (3.15) and (3.20) are sufficient to express all invariants $\mathcal{C}_{h_{1}, h_{2} ; l}^{(0)}$ and $\mathcal{D}_{h_{1}, h_{2} ; l}$, defined in (3.6) and (3.10), as linear combinations of $\mathcal{C}_{h_{1}, h_{2} ; l}^{(*)}$ with $\left(h_{1}, h_{2} ; l\right) \in \mathcal{P}_{k}^{(*)}$.

The proof of this theorem is given in Appendix D.2.

Given the existence of the consistent lattice theories (see the beginning of Section 3.2) this demonstrates that associativity of the OPE on the sphere and modular covariance of the torus one-point functions is sufficient to guarantee the consistency of the genus $g=2$ partition function, in agreement with the argument of Moore and Seiberg [1].

\subsection{Examples and the contracted Jacobi identities}

In the previous section, we have shown that the linear relations that are required for the consistency of the genus $g=2$ amplitudes follow from the associativity of the OPE on the sphere, as well as from the modular covariance of the torus one-point functions. However, it should be clear that the consistency at genus $g=2$ only checks some partial aspects of these requirements. To see this explicitly, it is useful to consider some simple examples.

3.3.1. Self-dual theories at $\boldsymbol{c}=\mathbf{2 4}$. For the case of $c=24$ we have $k=1$, and the set of free parameters labelled by (2.27) are

$$
C_{0,0 ; 0}^{(*)}, \quad C_{1,0 ; 0}^{(*)}, \quad C_{1,1 ; 0}^{(*)}
$$

At $h=1$ all states are Virasoro (quasi-)primary, and thus we need not distinguish between the quasiprimary functions $\mathcal{C}_{h_{1}, h_{2}}(x)$ and the actual four-point functions $C_{h_{1}, h_{2}}(x)$.

In terms of the sewn tori coordinate expansion, the free parameters are labelled by (2.33), and are thus given by

$$
\mathcal{D}_{0,0 ; 0}, \quad \mathcal{D}_{1,0 ; 0}, \quad \mathcal{D}_{1,1 ; 0}
$$

If we assume that our conformal field theory has a unique vacuum, it follows from (3.13) that we have

$$
\mathcal{D}_{0,0 ; 0}=1, \quad \mathcal{D}_{1,0 ; 0}=N_{1}, \quad \mathcal{D}_{1,1 ; 0}=N_{1}^{2}
$$

where $N_{1}$ is the number of currents. This reproduces the result of [8], where it was shown that for $c=24$ the genus $g=2$ amplitude is only a function 
312 Matthias R. Gaberdiel, Christoph A. Keller and Roberto Volpato

of $N_{1}$, namely

$$
W=\psi_{4}^{3}+\left(N_{1}-744\right) \psi_{12}+\left(N_{1}-744\right)\left(N_{1}+984\right) \chi_{12} .
$$

In particular, all the above invariants for a consistent self-dual conformal field theory at $c=24$ must satisfy a polynomial relation of degree two in the number of currents $N_{1}$. To see how this works explicitly, we note from (2.19) that the genus $g=2$ amplitude corresponding to $W$ has the expansion

$$
\begin{aligned}
\hat{Z}_{2}= & 1+N_{1}\left(p_{1}+p_{2}\right)+196884\left(p_{1}^{2}+p_{2}^{2}\right)+p_{1} p_{2} \\
& {\left[6 N_{1}+\frac{5 N_{1}^{2}}{6}+\left(-4 N_{1}+\frac{N_{1}^{2}}{12}\right)\left(x+\frac{1}{x}\right)+N_{1}\left(x^{2}+\frac{1}{x^{2}}\right)\right]+\cdots }
\end{aligned}
$$

To obtain the invariants $C_{1,1 ; l}^{(0)}$ we expand the coefficient of $p_{1} p_{2}$ as

$$
\begin{aligned}
(x-1)^{-2} C_{1,1}(x)= & \frac{N_{1}}{x^{2}}+\frac{-2 N_{1}+\frac{N_{1}^{2}}{12}}{x}+\left(N_{1}+N_{1}^{2}\right) \\
& +2 N_{1}^{2} x+3 N_{1}^{2} x^{2}+\cdots,
\end{aligned}
$$

thus leading to

$$
\begin{array}{ll}
\mathcal{C}_{1,1 ; 0}^{(0)}=N_{1}, & \mathcal{C}_{1,1 ; 1}^{(0)}=-2 N_{1}+\frac{N_{1}^{2}}{12}, \\
\mathcal{C}_{1,1 ; 2}^{(0)}=N_{1}+N_{1}^{2}, & \mathcal{C}_{1,1 ; s}^{(0)}=(s-1) N_{1}^{2}, \quad s \geq 3 .
\end{array}
$$

On the other hand, it follows from the general structure of a conformal field theory that the currents satisfy the commutation relations

$$
\left[J_{m}^{a}, J_{n}^{b}\right]=i f_{a b c} J_{m+n}^{c}+m \delta_{a b} \delta_{m,-n},
$$

where $f_{a b c}$ are the structure constants, and we have normalized the currents (so that the central extension term is proportional to $\mathrm{m}$, rather than $\mathrm{km}$.) 
In terms of these structure constants one then finds

$$
\begin{aligned}
\mathcal{C}_{1,1 ; 0}^{(0)} & =\sum_{a} \operatorname{Tr}_{\mathcal{H}_{1}}\left(J_{-1}^{a} J_{1}^{a}\right)=N_{1}, \\
\mathcal{C}_{1,1 ; 1}^{(0)} & =\sum_{a} \operatorname{Tr}_{\mathcal{H}_{1}}\left(J_{0}^{a} J_{0}^{a}\right)=-\sum_{a b c} f_{a b c} f_{a c b}, \\
\mathcal{C}_{1,1 ; 2}^{(0)} & =\sum_{a} \operatorname{Tr}_{\mathcal{H}_{1}}\left(J_{1}^{a} J_{-1}^{a}\right) \\
& =\sum_{a} \operatorname{Tr}_{\mathcal{H}_{1}}\left(J_{-1}^{a} J_{1}^{a}\right)+\sum_{a} \operatorname{Tr}_{\mathcal{H}_{1}}\left(\left[J_{1}^{a}, J_{-1}^{a}\right]\right)=N_{1}+N_{1}^{2}, \\
\mathcal{C}_{1,1 ; s}^{(0)} & =\sum_{a} \operatorname{Tr}_{\mathcal{H}_{1}}\left(J_{s-1}^{a} J_{-s+1}^{a}\right) \\
& =\sum_{a} \operatorname{Tr}_{\mathcal{H}_{1}}\left(\left[J_{s-1}^{a}, J_{-s+1}^{a}\right]\right)=(s-1) N_{1}^{2}, \quad(s \geq 3) .
\end{aligned}
$$

Thus we obtain the non-trivial identity

$$
\sum_{a b c} f_{a b c} f_{a b c}=\frac{N_{1}^{2}}{12}-2 N_{1}
$$

where we have used the total anti-symmetry of the structure constants (which follows from the associativity of the OPE).

The analysis of the previous section implies that this identity must follow from the associativity of the OPE, as well as the modular covariance of the one-point functions. To see how this goes we observe that modular covariance of the one-point functions implies that

$$
Z_{\phi}=0 \quad \text { for } \quad \phi=\sum_{a} J_{-1}^{a} J_{-1}^{a} \Omega-\frac{N_{1}}{12} L_{-2} \Omega
$$

since $\phi$ is a Virasoro primary state with $h=2$. Indeed, since $V_{0}(\phi) \Omega=0$ it follows that $Z_{\phi}$ must be holomorphic, but there are no holomorphic modular forms of weight two. In particular, this therefore implies that

$$
0=\operatorname{Tr}_{\mathcal{H}_{1}}\left(V_{0}(\phi)\right)=-\frac{N_{1}^{2}}{12}+\sum_{a} \operatorname{Tr}_{\mathcal{H}_{1}}\left(J_{0}^{a} J_{0}^{a}\right)+2 \sum_{a} \operatorname{Tr}_{\mathcal{H}_{1}}\left(J_{-1}^{a} J_{1}^{a}\right)
$$

which is just (3.30). 
314 Matthias R. Gaberdiel, Christoph A. Keller and Roberto Volpato

3.3.2. Self-dual theories at $c=48$ and Jacobi identities. For $c=$ 48 , i.e., $k=2$, the free parameters can, for example, be taken to be

$$
\mathcal{D}_{0,0 ; 0}, \quad \mathcal{D}_{1,0 ; 0}, \quad \mathcal{D}_{1,1 ; 0}, \quad \mathcal{D}_{2,0 ; 0}, \quad \mathcal{D}_{2,1 ; 0}, \quad \mathcal{D}_{2,2 ; 0}, \quad \mathcal{D}_{1,1 ; 1}, \quad \mathcal{D}_{2,2 ; 2}
$$

or

$$
\mathcal{C}_{0,0 ; 0}^{(0)}, \quad \mathcal{C}_{1,0 ; 1}^{(0)}, \quad \mathcal{C}_{1,1 ; 2}^{(0)}, \quad \mathcal{C}_{2,0 ; 2}^{(0)}, \quad \mathcal{C}_{2,1 ; 3}^{(0)}, \quad \mathcal{C}_{2,2 ; 4}^{(0)}, \quad \mathcal{C}_{1,1 ; 1}^{(0)}, \quad \mathcal{C}_{2,2 ; 2}^{(0)}
$$

For simplicity let us assume that the theory does not have any currents, $N_{1}=0$, and that the vacuum is unique. Then most of the above coefficients are zero, and the only non-trivial parameters are

$$
\begin{aligned}
& \mathcal{D}_{0,0 ; 0}=1, \quad \mathcal{D}_{1,0 ; 0}=0, \quad \mathcal{D}_{1,1 ; 0}=0, \quad \mathcal{D}_{2,0 ; 0}=N_{2}, \\
& \mathcal{D}_{2,1 ; 0}=0, \quad \mathcal{D}_{2,2 ; 0}=N_{2}^{2}, \quad \mathcal{D}_{1,1 ; 1}=0, \quad \mathcal{D}_{2,2 ; 2}=d,
\end{aligned}
$$

as follows from (3.13). Thus, there are only two independent parameters, namely $N_{2}$ and $d$. In terms of the $\mathcal{C}^{(0)}$ parameters, the only non-trivial invariants are

$$
\begin{gathered}
\mathcal{C}_{0,0 ; 0}^{(0)}=\operatorname{Tr}_{\mathcal{H}_{0}}(1)=1, \quad \mathcal{C}_{2,0 ; 2}^{(0)}=\operatorname{Tr}_{\mathcal{H}_{2}}(1)=N_{2}, \\
\mathcal{C}_{2,2 ; 4}^{(0)}=\sum_{j} \operatorname{Tr}_{\mathcal{H}_{2}}\left(\hat{W}_{2}^{j} \hat{W}_{-2}^{j}\right)=b_{1}, \quad \mathcal{C}_{2,2 ; 2}^{(0)}=\sum_{j} \operatorname{Tr}_{\mathcal{H}_{2}}\left(\hat{W}_{0}^{j} \hat{W}_{0}^{j}\right)=b_{2} \equiv b,
\end{gathered}
$$

where $N_{2}=\operatorname{dim}\left(\mathcal{H}_{2}\right)$, and the $\hat{W}^{j}$ are an orthonormal basis for the states at $h=2$. It follows that both $b_{1}$ and $b_{2}$ must be a function of $N_{2}$ and $d$; we can then take the independent parameters also to be $N_{2}$ and $b$.

For a theory without currents we know that the commutation relations of the $W_{m}^{i}$ modes take the form (see, for example, [24])

$$
\left[W_{m}^{i}, W_{n}^{j}\right]=\frac{c}{12} \delta^{i j} m\left(m^{2}-1\right) \delta_{m,-n}+(m-n) h_{i j k} W_{m+n}^{k}+i g_{\alpha}^{i j} V_{m+n}^{\alpha},
$$

where $h_{i j k}$ and $g_{\alpha}^{i j}$ are structure constants, and $V_{n}^{\alpha}$ denotes a basis for the space of states at $h=3$. (The modes $W_{m}^{j}$ and $\hat{W}_{m}^{j}$ differ only by the normalization factor $\sqrt{2 / c}$.) With these definitions we can then calculate directly 
the four-point functions

$$
\begin{aligned}
\sum_{n, j} x^{-n-2} \operatorname{Tr}_{\mathcal{H}_{2}}\left(\hat{W}_{-n}^{j} \hat{W}_{n}^{j}\right) & =\frac{2}{c} \sum_{n, j} x^{-n-2} \operatorname{Tr}_{\mathcal{H}_{2}}\left(W_{-n}^{j} W_{n}^{j}\right) \\
& =\frac{N_{2}}{x^{4}}+\frac{8 H_{1}}{c x^{2}}+\frac{8 H_{2}}{c x}+N_{2}+N_{2}^{2}+\frac{16 H_{2}}{c} \cdots,
\end{aligned}
$$

as well as

$$
\begin{aligned}
\sum_{n, j} x^{-n-2} \operatorname{Tr}_{\mathcal{H}_{3}}\left(\hat{W}_{-n}^{j} \hat{W}_{n}^{j}\right) & =\frac{2}{c} \sum_{n, j} x^{-n-2} \operatorname{Tr}_{\mathcal{H}_{3}}\left(W_{-n}^{j} W_{n}^{j}\right) \\
& =\frac{4 N_{2}}{x^{5}}+\frac{\frac{8}{c} H_{1}+\frac{4}{c^{2}} G}{x^{3}}+\cdots,
\end{aligned}
$$

where we have defined

$$
H_{1}=\sum_{i j k} h_{i j k} h_{i j k}, \quad H_{2}=\sum_{i j k} h_{i i j} h_{j k k}, \quad G=\sum_{i j \alpha} g_{\alpha}^{i j} g_{\alpha}^{i j}
$$

As an aside we should note that some of these coefficients can obviously be calculated in different ways which agree, however, if the Jacobi identity is satisfied. For example, the coefficient proportional to $x^{-1}$ in (3.39) was calculated as

$$
\sum_{j} \operatorname{Tr}_{\mathcal{H}_{2}}\left(\hat{W}_{1}^{j} \hat{W}_{-1}^{j}\right)=\frac{2}{c} \sum_{j} \operatorname{Tr}_{\mathcal{H}_{2}}\left(\left[W_{1}^{j}, W_{-1}^{j}\right]\right)=\frac{8}{c} \sum_{i j k} h_{j j i} h_{k k i} .
$$

However, we could have also evaluated it directly, by first applying $W_{-1}^{j}$ to the states $W_{-2}^{k} \Omega$ using

$$
W_{-1}^{j} W_{-2}^{k} \Omega=h_{j k m} W_{-3}^{m} \Omega+i g_{\alpha}^{j k} V_{-3}^{\alpha} \Omega
$$

and then evaluating $W_{1}^{j}$ on the resulting state and taking the trace. This would have led to a different expression for the coefficient of $x^{-1}$. Requiring the two results to agree is precisely the identity (3.48) below, which is a consequence of the Jacobi identity.

On the other hand, we know from (3.37) that

$$
(x-1)^{-4} \mathcal{C}_{2,2}(x)=\frac{N_{2}}{x^{4}}+\frac{b}{x^{2}}+\frac{N_{2}^{2}}{6 x}+N_{2}+\frac{4 N_{2}^{2}}{3}+\frac{9 N_{2}^{2}}{2} x+\frac{32 N_{2}^{2}}{3} x^{2}+\cdots,
$$


316 Matthias R. Gaberdiel, Christoph A. Keller and Roberto Volpato

as well as

$$
\begin{aligned}
(x-1)^{-4} \mathcal{C}_{3,2}(x)= & \frac{4 N_{2}}{x^{5}}+\frac{N_{2}^{2}}{6 x^{3}}+\frac{15750720+240 b+369560 N_{2}}{x^{2}} \\
& +\frac{10746880 N_{2}+\frac{N_{2}^{2}}{6}}{x}+\cdots
\end{aligned}
$$

By comparing coefficients we can then obtain $H_{1}, H_{2}$ and $G$ as a function of $N_{2}$ and $b$; in particular, we have from the comparison of (3.39) and (3.44)

$$
H_{1}=\frac{c b}{8}, \quad H_{2}=\frac{c}{48} N_{2}^{2},
$$

while the comparison of (3.40) and (3.45) leads to

$$
G=\frac{c^{2}}{24} N_{2}^{2}-\frac{c^{2}}{4} b
$$

For $c=48$ we then obtain the non-trivial relation

$$
G=96\left(H_{2}-H_{1}\right)
$$

As pointed out above, this relation is actually a consequence of the Jacobi identity of (3.38). Indeed, by considering the identity $\left[W_{n}^{i},\left[W_{m}^{j}, W_{l}^{k}\right]\right]+$ cycl. $=0$, we find the constraint

$$
\frac{1}{2 c} g_{\alpha}^{j t} g_{\alpha}^{k i}-h_{j k s} h_{i s t}+h_{i j s} h_{k s t}=0 .
$$

Contracting with $\delta_{j k} \delta_{i t}$ we get

$$
\frac{1}{2 c}\left(g_{\alpha}^{j i}\right)^{2}-h_{j j s} h_{i i s}+h_{i j s} h_{i j s}=\frac{1}{2 c} G+H_{1}-H_{2}=0 .
$$

Thus the consistency at genus $g=2$ tests the aspects of the Jacobi identity. It is clear, however, that we only get identities between fully contracted expressions, i.e., only between the specialized expressions (3.50). Thus, the genus $g=2$ amplitudes give us only partial access to the full Jacobi identity (3.49). This remark will be further explored in Section 5.

\section{Application to the extremal ansatz}

Up to now we have studied the constraints a conformal field theory has to satisfy in order to lead to a consistent genus two amplitude. In particular, we have seen that consistency at genus two is guaranteed if the OPE 
on the sphere is associative and the torus one-point functions are modular covariant. However, as is apparent from the arguments of Section 3.2.2, these conditions are sufficient, but certainly not necessary. Indeed, it is clear from the last example above that only certain aspects of the associativity constraints are actually relevant for the genus two consistency analysis.

It is therefore an interesting question to ask the converse question: how much information do we need about a conformal field theory (be it ultimately consistent or not) in order to construct a (seemingly) consistent genus two amplitude. This question is of particular relevance in the context of the extremal theories originally proposed in [2] as the dual conformal field theory of pure gravity on $\mathrm{AdS}_{3}[2,3]$. This proposal was subsequently modified for chiral massive gravity in [25], but there have been arguments to suggest that chiral gravity is actually logarithmic [26-30], in which case the dual conformal field theory would not be extremal in the above sense. There is also a conformal field theory argument that suggests that such extremal theories cannot exist for large values of the central charge $[9,10,31]$.

The proposed extremal conformal field theories are self-dual meromorphic conformal field theories at $c=24 k$. Up to conformal weight $h=k$ their spectrum only consists of Virasoro descendants of the vacuum. In order for the torus partition function to be modular invariant, the theory has to have additional primary states. These appear only at conformal dimension $h>k$, and their number (and conformal dimension) is uniquely fixed by the requirement that the torus partition function is modular invariant.

In the context of the $\mathrm{AdS}_{3} / \mathrm{CFT}_{2}$ duality, the gravity calculation gives in principle also access to the higher genus partition functions, and one may ask whether their existence will be evidence in favour of the consistency of these theories. In fact, for $k=2$ and $k=3$, the explicit genus $g=2$ partition function was constructed in [2] and [4-6]. As we have seen in Section 3, the consistency of the genus two amplitudes checks certain aspects of the associativity of the OPE, as well as the modular covariance of the torus one-point functions. One may therefore expect that the existence of these $g=2$ amplitudes provides a non-trivial consistency check on the existence of these proposed theories. Unfortunately, as we shall see in the following, this is not the case.

In order to explain more precisely what we mean by this statement, it is instructive to consider a slightly more general situation. Suppose that the spectrum of the putative self-dual chiral conformal field theory is of the form

$$
\mathcal{H}=\mathcal{H}^{(0)} \oplus \mathcal{R}, \quad \mathcal{R} \subset \bigoplus_{n \geq B} \mathcal{H}_{n}
$$


where $\mathcal{H}^{(0)}$ is the vacuum representation of some consistent chiral algebra $\mathcal{A}$, and $\mathcal{R}$ is a (reducible) representation of $\mathcal{A}$. Here $\mathcal{H}_{n}$ is the finite dimensional eigenspace of $\mathcal{H}$ with $L_{0}$-eigenvalue $n$, and we assume that the $L_{0}$ spectrum of $\mathcal{H}$ is bounded from below by zero, with a unique state, the vacuum, at conformal weight $h=0$. Furthermore, we assume that the chiral algebra $\mathcal{A}$ contains the Virasoro algebra at $c=24 k$. On the other hand, the states in $\mathcal{R}$ all have conformal weight $h \geq B$, and we do not (a priori) assume that we know anything about the OPE involving two fields from $\mathcal{R}$. In the example of the extremal theories, $\mathcal{A}$ would be the Virasoro algebra, and the states in $\mathcal{R}$ describe the additional states whose conformal dimensions are bigger or equal than $B=k+1$.

By assumption, the full space $\mathcal{H}$ is a representation of the chiral algebra $\mathcal{A}$, and thus the torus one-point functions

$$
Z_{\phi}=\operatorname{Tr}_{\mathcal{H}}\left(V_{0}(\phi) q^{L_{0}-k}\right)
$$

with $\phi \in \mathcal{H}^{(0)}$, are determined by our ansatz (4.1). If $\phi$ has $L_{[0]}$ eigenvalue $h$, then we know on general grounds that $Z_{\phi}$ must be an element of $M_{h, k}$, see the discussion in Section 3.1.2. Thus consistency at genus one already requires that these torus one-point functions are modular invariant. Provided that this is the case (as we shall from now on assume), the relations that come from the modular covariance of the torus one-point functions (see Section 3.2.2) are therefore automatically satisfied.

In order to understand what constraints the genus two analysis implies we therefore consider the other invariants we introduced above, namely $\mathcal{C}_{h_{1}, h_{2} ; l}^{(0)}$ and $\mathcal{D}_{h_{1}, h_{2} ; l}$. Since we do not know anything about the OPEs involving general states in $\mathcal{H}$, we shall only consider the invariants that can be defined and computed given the ansatz (4.1). These include, in particular,

$$
\mathcal{C}_{h_{1}, h_{2} ; l}^{(0)}=\sum_{\phi, \psi \in \mathcal{H}_{h_{2}}^{q p}} G_{\phi \psi}^{-1} \operatorname{Tr}_{\mathcal{H}_{h_{1}}}\left(V_{l-h_{1}}(\phi) V_{h_{1}-l}(\psi)\right), \quad h_{2}<B
$$

since for $h_{2}<B$ the relevant states $\phi$ and $\psi$ are part of the chiral algebra, and we know, by assumption, how they act on the full space $\mathcal{H}$. Similarly, regarding the invariants $\mathcal{D}_{h_{1}, h_{2} ; l}$, we can calculate those for which $h_{2}<B$ or $2 l<B$. Indeed, in either case we can restrict the sum over $\mathcal{H}_{[2 l]}$ in

$$
\mathcal{D}_{h_{1}, h_{2}, l}=\sum_{\phi, \psi \in \mathcal{H}_{[2 l]}} G_{\phi \psi}^{-1} \operatorname{Tr}_{\mathcal{H}_{h_{1}}}\left(V_{0}(\phi)\right) \operatorname{Tr}_{\mathcal{H}_{h_{2}}}\left(V_{0}(\psi)\right), \quad h_{2}<B \text { or } 2 l<B,
$$


to states in $\mathcal{H}^{(0)}$. This is obvious for the case that $2 l<B$. For $h_{2}<B$ note that if $\psi$ is not an $\mathcal{A}$ descendant of the vacuum, $\operatorname{Tr}_{\mathcal{H}_{h_{2}}} V_{0}(\psi)$ vanishes since $\mathcal{H}_{h_{2}}$ contains only vacuum descendants. Thus in either case only $\mathcal{A}$ descendants contribute, whose contributions we can compute. Thus, we may take (4.4), with the sum restricted to $\mathcal{H}_{[2 l]} \cap \mathcal{H}^{(0)}$, as the definition of $\mathcal{D}_{h_{1}, h_{2} ; l}$ for $h_{2}<B$ or $2 l<B$.

We conclude that there are infinitely many invariants, namely (4.3) and (4.4) for arbitrary $h_{1}$, that we can calculate from our ansatz. On the other hand, we know from the analysis of Section 2 that consistency at genus two implies that there are only finitely many free parameters, and thus that these invariants must satisfy infinitely many linear relations. It would thus seem that we get strong constraints from the consistency of the genus two amplitudes!

Unfortunately, this impression is somewhat deceptive. As we have seen in Section 3 the constraints that arise in this way test effectively aspects of the associativity of the OPE. However, since we only have access to the invariants of the form (4.3) with $h_{2}<B$, we will only be able to test the associativity of the OPE of the fields up to conformal weight $B$. But since we assumed that, up to conformal weight $B$, the conformal field theory consists just of elements in the chiral algebra $\mathcal{A}$ (for which associativity is assumed) all of these constraints will be automatically satisfied. More precisely we can prove the following:

Theorem 4.1. Suppose $\mathcal{H}$ is of the form (4.1) and all torus one-point functions $Z_{\phi}$ with $\phi \in \mathcal{H}^{(0)}$ are modular covariant. Then there exists a (not necessarily unique) modular form of degree $g=2$ and weight $12 k$, whose coefficients in the expansions (2.23) agree with (4.3) for $h_{2}<B$, and whose coefficients in the expansion (2.31) with either $h_{2}<B$ or $2 l<B$ agree with (4.4).

Proof. The proof is analogous to Theorem 3.1, but this time we are not allowed to assume that $\mathcal{H}$ is a vertex operator algebra. This means that we cannot assume that all identities (3.8), (3.14), (3.15) and (3.20) hold. However, we can still follow the same strategy as before: using those identities that still follow from the associativity of $\mathcal{A}$ and the modular covariance of the one-point functions (4.2), we can demonstrate that all invariants (4.3) and (4.4) can be expressed in terms of linear combinations of some $\mathcal{C}_{h_{1}, h_{2} ; l}^{(*)}$ with $\left(h_{1}, h_{2} ; l\right) \in \mathcal{P}_{k}^{(*)}$. Given our previous analysis we know, on the other hand, that these relations are the same as those that arise from the modular covariance at $g=2$, and hence the result follows. 
To see this in more detail we first note that the limits (2.20) still hold since the $L_{0}$ spectrum is bounded by assumption. Next, using that all operators in $\mathcal{A}$ are local, we write

$$
\begin{aligned}
\left(x^{-1}-1\right)^{2 h_{2}} \operatorname{Tr}_{\mathcal{H}_{h_{1}}}\left(V(\psi, 1) V\left(\phi, x^{-1}\right)\right) \\
\quad=x^{-2 h_{2}}(x-1)^{2 h_{2}} \operatorname{Tr}_{\mathcal{H}_{h_{1}}}\left(V\left(\phi, x^{-1}\right) V(\psi, 1)\right) \\
\quad=x^{-2 h_{2}}(x-1)^{2 h_{2}} \sum_{n} x^{n+h_{2}} \operatorname{Tr}_{\mathcal{H}_{h_{1}}}\left(\phi_{n} \psi_{-n}\right) \\
\quad=(x-1)^{2 h_{2}} \operatorname{Tr}_{\mathcal{H}_{h_{1}}}(V(\phi, 1) V(\psi, x)),
\end{aligned}
$$

from which $(2.22 \mathrm{~b})$ follows after summing over all states in $\mathcal{H}_{h_{2}}$. We can thus again define invariants $\mathcal{C}_{h_{1}, h_{2} ; l}^{(*)}, h_{2}<B$, which are related to the $\mathcal{C}_{h_{1}, h_{2} ; l}^{(0)}$ as in (3.8). Equation (3.15) is still valid for $h_{2}<B$ because it only makes use of the cyclicity of the trace. Furthermore, as shown in Appendix D.1, (3.14) is valid for $h_{2}<B$. Using the same arguments as in the proof of Lemma 1 (see Appendix D.2) it then follows that the invariants (4.3) and the invariants (4.4) for $h_{2}<B$ can be correctly reproduced by a suitable choice of the free parameters in

$$
S=\left\{\left(h_{1}, h_{2} ; l\right) \in \mathcal{P}_{k}^{(*)}: h_{2}<B\right\} .
$$

It remains to prove that the modular form can be consistently chosen in such a way that also the invariants $\mathcal{D}_{h_{1}, h_{2} ; l}$ with $2 l<B \leq h_{2}$ are correctly reproduced. This works because the only consistency checks of those invariants come essentially from the modular covariance of the onepoint functions. More precisely, since by assumption the one-point functions are modular covariant, we can use (3.20) to express all such invariants by $\mathcal{D}_{h_{1}^{\prime}, h_{2}^{\prime} ; l}$ with $h_{2}^{\prime} \leq h_{1}^{\prime} \leq k+l-w_{l}$. If all $h_{2}^{\prime}<B$, we know from the arguments given above that the invariants are consistent with all other invariants in (4.3) and (4.4). If on the other hand, some $h_{2}^{\prime}$ appears with $h_{2}^{\prime} \geq B$, then $2 l<B$ implies $\left(h_{1}^{\prime}, h_{2}^{\prime} ; l\right) \in \mathcal{P}_{k}^{(*)}$, and thus we can simply fix the correspond$\operatorname{ing} \mathcal{C}_{h_{1}^{\prime}, h_{2}^{\prime} ; l}^{(*)}$. Thus the only effect of the invariants (4.4) with $2 l<B \leq h_{2}$ is to fix the free parameters

$$
S^{\prime}=\left\{\left(h_{1}, h_{2} ; l\right) \in \mathcal{P}_{k}^{(*)}: 2 l<B \leq h_{2}\right\} .
$$

This completes the proof.

Note that in general $S \cup S^{\prime}$ is a proper subset of $\mathcal{P}_{k}^{(*)}$, in which case, the genus $g=2$ amplitude is not uniquely fixed by the information on $\mathcal{H}$, i.e., by 
(4.3) and (4.4). (An example of this is described below.) Finally, we observe that the assumptions of the theorem can be weakened, because modular covariance of $Z_{\phi}$ is only needed for all $\phi \in \mathcal{H}^{(0)}$ with conformal weight less than $2 B$.

\subsection{The extremal ansatz}

The above proposition can be directly applied to the proposed extremal theories. As was already mentioned before, in this context $\mathcal{A}$ is the Virasoro algebra, and $B=k+1$. The proposition then implies that a consistent genus two amplitude can be constructed provided that the torus one-point functions $Z_{\phi}$ are modular invariant (with the appropriate modular weight).

For the case at hand, this latter condition is actually straightforward to check. Because the chiral algebra $\mathcal{A}$ is just the Virasoro algebra, the only states $\phi$ that appear are Virasoro descendants of the vacuum. Using the recursion relations of Zhu [20] (see also [10]) it is clear that each such onepoint function can be written as a modular covariant differential operator $D_{q}^{(\phi)}$ acting on the vacuum torus amplitude,

$$
Z_{\phi}(q) \equiv \operatorname{Tr}_{\mathcal{H}}\left(V_{0}(\phi) q^{L_{0}-k}\right)=D_{q}^{(\phi)} \operatorname{Tr}_{\mathcal{H}}\left(q^{L_{0}-k}\right), \quad \phi=L_{[-2]}^{n_{2}} L_{[-3]}^{n_{3}} \cdots \Omega
$$

For example, for $\phi=L_{[-2]} \Omega$, we have

$$
Z_{L_{[-2]} \Omega}(q) \equiv(2 \pi i)^{2} \operatorname{Tr}_{\mathcal{H}}\left(\left(L_{0}-k\right) q^{L_{0}-k}\right)=(2 \pi i)^{2} q \frac{d}{d q} \operatorname{Tr}_{\mathcal{H}}\left(q^{L_{0}-k}\right),
$$

so that

$$
D_{q}^{\left(L_{[-2]} \Omega\right)}=(2 \pi i)^{2} q \frac{d}{d q} .
$$

If $\phi$ has $L_{[0]}$ eigenvalue $h$ with $h$ even — for $h$ odd, the torus one-point function, and thus the differential operator, vanishes identically — the differential operator is of order $\frac{h}{2}$ and of modular weight $h$. Thus the modular covariance of the one-point functions $Z_{\phi}$ is a direct consequence of the modular invariance of the torus vacuum amplitude (which is satisfied by construction).

This argument therefore demonstrates that a consistent genus $g=2$ amplitude, satisfying all relations (4.3) with $h_{2} \leq k$, exists for all $k$. This conclusion is obviously in agreement with the results for $k=2$ and $k=3$ 
in $[2,4]$. However, it also shows that one is not actually testing any nontrivial consistency conditions of the extremal ansatz in this way. In fact, the theorem shows that the same would have been true for any ansatz satisfying the above conditions.

\subsubsection{Explicit examples: the extremal ansatz for low values of $\boldsymbol{k}$.}

It is maybe instructive to see how the general machinery works out for these examples. Let us first consider the case with $k=2(c=48)$. The extremal ansatz is a special case of the example studied in Section 3.2.2. Indeed, if at $h=2$ we only have the Virasoro field, the parameters $N_{2}$ and $B$ take the form

$$
N_{2}=1, \quad B=\frac{2}{c} \operatorname{Tr}_{\mathcal{H}_{2}}\left(L_{0}^{2}\right)=\frac{8}{c}=\frac{1}{6}
$$

Thus it follows from (3.46) that $H_{1}=1=H_{2}=1$, which is indeed in agreement with the definition in (3.41).

For $k=3(c=72)$ the analysis is similar. In this case the free parameters may be taken to be $N_{1}, N_{2}, N_{3}$, as well as

$$
\mathcal{C}_{1,1 ; 1}^{(0)}, \quad \mathcal{C}_{2,1 ; 2}^{(0)}, \quad \mathcal{C}_{2,2 ; 3}^{(0)}, \quad \mathcal{C}_{2,2 ; 2}^{(0)}, \quad \mathcal{C}_{3,2 ; 3}^{(0)}, \quad \mathcal{C}_{3,3 ; 4}^{(0)}, \quad \mathcal{C}_{3,3 ; 3}^{(0)}
$$

All of these invariants can be computed from the extremal ansatz, so that the genus 2 partition function is uniquely determined. We have also checked explicitly that the low-lying invariants in (4.3) and (4.4) are indeed correctly reproduced (as our general argument predicts). We have also done the same analysis at $k=4$.

Something new happens at $k=5$ since there is a modular form $\chi_{10}^{6}$ of weight $12 k=60$ whose leading term is of order $p_{1}^{6} p_{2}^{6}$ in the expansion $(2.16)$. Its coefficient is associated with the invariant

$$
\mathcal{C}_{6,6 ; 6}^{(0)}=\sum_{\phi, \psi \in \mathcal{H}_{6}^{q p}} G_{\phi \psi}^{-1} \operatorname{Tr}_{\mathcal{H}_{6}}\left(V_{0}(\phi) V_{0}(\psi)\right)
$$

that cannot be determined directly from the extremal ansatz. Thus for $k \geq 5$ the extremal ansatz does not specify the genus $g=2$ partition function uniquely, ${ }^{1}$ and there is a whole vector space of genus $g=2$ partition functions that reproduce correctly all computable invariants.

\footnotetext{
${ }^{1}$ This corrects a statement in [4].
} 


\section{Remarks about higher genus}

Let us close this paper by coming back to the question that was raised in the Introduction. Recall that according to an old idea of Friedan and Shenker [7], the higher genus partition functions determine a conformal field theory uniquely. Assuming that this idea is correct, then constructing all higher genus vacuum amplitudes would be a way of defining, say, the dual conformal field theory of some gravity theory on $\mathrm{AdS}_{3}$. The interesting question would then be: what consistency conditions apart from modular invariance do the higher genus vacuum amplitudes have to satisfy in order to define a consistent conformal field theory?

Obviously, it is in practice quite hard to calculate these higher genus amplitudes explicitly (see however [6]), but one could attempt to construct the higher genus amplitudes by symmetry principles. For example, one could specify the algebra of low-lying states of the conformal field theory, and simply attempt to find modular invariant higher genus amplitudes that satisfy all appropriate factorization conditions with respect to these low-lying states. At genus $g=2$, Theorem 4.1 shows that this will be possible provided that one chooses $\mathcal{A}$ and $\mathcal{R}$ appropriately (where the required conditions are rather weak). While we have not done any detailed analysis at higher genus (see however Section 5.2), it seems plausible that a similar statement to Theorem 4.1 could also hold at higher genus.

Suppose then that we have found such a family of higher genus amplitudes, say for the extremal ansatz. Would this prove that the extremal theories exist? As we have seen in Section 3, modular invariance at genus $g=2$ implies a large number of relations between different expansion coefficients which, in terms of the conformal field theory, translate into the statement that certain quadratic expressions in the structure constants have to be equal. These in turn are a consequence of the Jacobi identity of the $\mathcal{W}$-algebra, i.e., of the associativity of the OPE, see Section 4.1. One would similarly expect that higher genus constraints will give rise to relations that, from the point of view of the underlying conformal field theory, involve higher powers of these structure constants (which again should be implied by the associativity of the OPE). Consistency at arbitrary genus would thus probably imply that all possible contractions of the Jacobi identities will be satisfied. Would this be sufficient to reconstruct the Jacobi identities themselves, i.e., to prove associativity of the OPEs?

The answer to this question is no - for a rather simple reason. To explain this, let us briefly return to the example of Section 3.3.2. The higher genus vacuum amplitudes give us access to fully contracted polynomials 
of the structure constants, such as $H_{1}, H_{2}$ and $G$ in (3.41). However, it is not guaranteed that we can find individual structure constants $h_{i j k}$ and $g_{\alpha}^{i j}$ (that must be $N_{2} \times N_{2} \times N_{2}$ tensors and $N_{2} \times N_{2} \times N_{3}$ tensors, respectively), whose contractions reproduce the given values of $H_{1}, H_{2}$ and $G$. (Here $N_{h}$ denotes the number of states at conformal weight $h$, which can be read off from the torus amplitude.) We shall demonstrate in the following that this is a non-trivial consistency condition which does not seem to be implied by the modular invariance of the higher genus amplitudes. Thus a family of modular invariant higher genus vacuum amplitudes can only define a consistent conformal field theory if this consistency condition is satisfied. It is natural to speculate that this will be the only additional consistency condition beyond modular invariance.

\subsection{Obstructions at $c=24$}

The simplest example where the above consistency condition is non-trivial appears for the self-dual theories at $c=24$. As was shown in [8], at $c=24$ consistent $g=1,2,3,4$ vacuum amplitudes (that have the correct modular and factorization properties) can be found for any number of currents $N_{1}$. However, it is believed that only 71 consistent conformal field theories exist at $c=24$. In particular, no theories exist for $0<N_{1}<24$ [32]. Unless $N_{1}$ is one of the special values for which a consistent theory exists, there must be an obstruction towards reconstructing the theory from the vacuum amplitudes. Obviously, it is conceivable that this obstruction will manifest itself in that one cannot find vacuum amplitudes (with the correct modular and factorisation properties) for $g \geq 5$, but this seems unlikely to us. Instead we believe that the obstruction appears in that one cannot reproduce the coefficients of the modular forms in terms of contracted structure constants. In fact, we can see this obstruction very explicitly in some simple examples, as we shall now show.

In the following the states at $h=1$ will play an important role. One knows on general grounds (see, e.g., [33]) that the modes of these fields satisfy the commutation relations

$$
\left[J_{m}^{a}, J_{n}^{b}\right]=m \kappa^{a b} \delta_{m,-n}+i f_{c}^{a b} J_{m+n}^{c}
$$

where $\kappa^{a b}$ is non-singular with inverse $\kappa_{a b}$. Furthermore, $f_{c}^{a b}$ is antisymmetric in $a \leftrightarrow b$. 
As is explained in [8] the number of currents $N_{1}$ fixes the genus $g=2,3,4$ amplitudes uniquely. In turn, one can read off from this that (at $c=24$ )

$$
\operatorname{Tr}_{\mathcal{H}_{1}}\left(\left(\kappa_{a b} J_{0}^{a} J_{0}^{b}\right)^{l}\right)=N_{1}\left(\frac{N_{1}}{24}-1\right)^{l}, \quad \text { for } l=1,2,3 .
$$

We shall use this relation in the following to show that the theories with $N_{1}=1,2$ (that do not arise among the 71 theories of [32]) are in fact inconsistent.

5.1.1. The theory with $N_{1}=1$. The simplest case is the theory with $N_{1}=1$. It is immediately clear that this theory is inconsistent since at $N_{1}=1$ the Lie algebra (5.1) is necessarily abelian (since $f_{c}^{a b}$ is antisymmetric in $a \leftrightarrow b$ and must hence vanish). As a consequence, the trace in (5.2) vanishes for $l=1,2,3$, which disagrees with the right-hand side.

5.1.2. The theory with $\boldsymbol{N}_{\mathbf{1}}=\mathbf{2}$. The situation with $N_{1}=2$ is more interesting. Since $\kappa_{a b}$ is symmetric, we may choose a basis of $\mathcal{H}_{1}$ that diagonalizes $\kappa_{a b}$, and set

$$
\kappa_{11}=\epsilon_{1}, \quad \kappa_{22}=\epsilon_{2}, \quad i f_{1}^{12}=\alpha, \quad i f_{2}^{12}=\beta .
$$

Then, $J_{0}^{1}$ and $J_{0}^{2}$ in the adjoint representation correspond to the matrices

$$
J_{0}^{1}=\left(\begin{array}{cc}
0 & \alpha \\
0 & \beta
\end{array}\right) \quad \text { and } \quad J_{0}^{2}=\left(\begin{array}{ll}
-\alpha & 0 \\
-\beta & 0
\end{array}\right)
$$

and we find

$$
\kappa_{a b} J_{0}^{a} J_{0}^{b}=\left(\begin{array}{cc}
\epsilon_{2} \alpha^{2} & \epsilon_{1} \alpha \beta \\
\epsilon_{2} \alpha \beta & \epsilon_{1} \beta^{2}
\end{array}\right)
$$

The eigenvalues of this matrix are 0 and $\epsilon_{2} \alpha^{2}+\epsilon_{1} \beta^{2}$, so that (5.2) becomes

$$
\operatorname{Tr}_{\mathcal{H}_{1}}\left(\left(\kappa_{a b} J_{0}^{a} J_{0}^{b}\right)^{l}\right)=\left(\epsilon_{2} \alpha^{2}+\epsilon_{1} \beta^{2}\right)^{l}=2(-11 / 12)^{l} \quad \text { for } l=1,2,3 .
$$

It is easy to see that this does not have any solution.

One can similarly analyse the situation with $N_{1}=3$, but there the constraints from $l=1,2,3$ (i.e., from genus $g \leq 4$ ) are not sufficient to lead to a contradiction. This is not surprising: at $N_{1}=3$ there are four free parameters, namely the three eigenvalues of $\kappa$, as well as the one totally antisymmetric structure constant $f^{123}=f_{c}^{12} \kappa^{3 c}$. On the other hand, (5.2) only 
gives rise to three equations, and a solution can be found. Indeed, one can take the currents to define an affine $\operatorname{su}(2)$ algebra at level $k$ with $k=-\frac{16}{7}$ since then

$$
\kappa_{a b} J_{0}^{a} J_{0}^{b}=-\frac{7}{8} \mathbf{1}_{3 \times 3} \quad \text { on } \mathcal{H}_{1} .
$$

This then solves (5.2). However, one would expect that this ansatz will not be compatible with the vacuum amplitudes at $g \geq 5$.

\subsection{Polynomial constraints from modular invariance}

The analysis of the previous section shows that not every family of modular invariant genus $g$ amplitudes defines a consistent conformal field theory. Indeed, the main additional condition seems to be that there is a solution for the individual structure constants such that the contracted powers reproduce the expansion coefficients of the corresponding modular amplitudes. Obviously, this condition only becomes powerful at sufficiently high genus when the number of equations for the contracted structure constants exceeds the number of (unknown) structure constants.

To be more specific, let $Z_{g}$ be a modular form of genus $g$ and weight $12 k$, and consider a series expansion of $Z_{g}$ in $3 g-3$ suitable parameters $t_{1}, \ldots, t_{3 g-3}$

$$
Z_{g}=\sum_{h_{1}, \ldots, h_{3 g-3}} C_{h_{1}, \ldots, h_{3 g-3}} t_{1}^{h_{1}} \cdots t_{3 g-3}^{h_{3 g-3}} .
$$

More precisely, let us consider a sphere with $2 g$ punctures and decompose it into a set of pair of pants, i.e., into $2 g-2$ spheres with three punctures each. We then connect the punctures to obtain the $g$ handles of the surface, and take the $t_{i}$ to parametrize the $3 g-3$ different tubes. For example, the possible decompositions of a genus 2 surface are shown in figure 3 . As we shall see below, the number of pant decompositions grows very quickly with $g$.

If $Z_{g}$ is the genus $g$ partition function of a conformal field theory, then for every pant decomposition, each of the coefficients $C_{h_{1}, \ldots, h_{3 g-3}}$ equals a contracted combination of structure constants of the underlying conformal field theory. (Different pant decompositions of a Riemann surface correspond to different ways of contracting the indices.) Alternatively, instead of working with structure constants, we can also consider directly the threepoint functions of the conformal field theory. In this language the coefficients $C_{h_{1}, \ldots, h_{3 g-3}}$ must then equal contracted products of three-point functions 

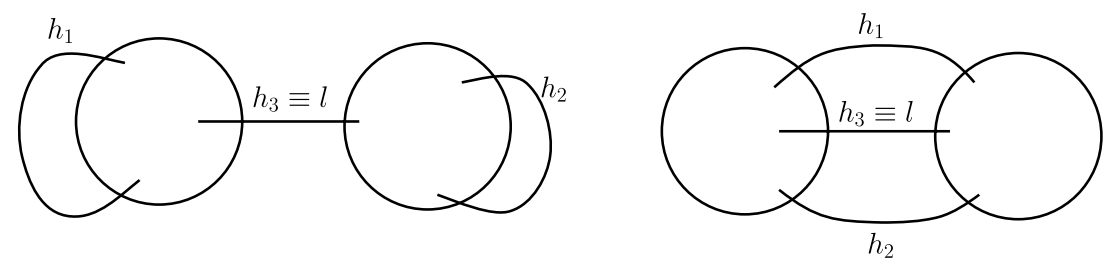

Figure 3: The two possible pants decompositions of a genus 2 surface. They correspond to the expansions in the coordinates $q_{1}, q_{2}, \epsilon$ (left) and $p_{1}, p_{2}, x$ (right), and the associated invariants are $\mathcal{D}_{h_{1}, h_{2} ; l}$ (left) and $\mathcal{C}_{h_{1}, h_{2} ; l}^{(0)}$ (right).

where the relevant fields have conformal weights $h_{1}, \ldots, h_{3 g-3}$. For the following it is convenient to define, for each fixed $L$, the set

$$
I_{L}(g):=\left\{\left(h_{1}, \ldots, h_{3 g-3}\right): \quad 0 \leq h_{1}, \ldots, h_{3 g-3}<L\right\}
$$

Then the coefficients $C_{h_{1}, \ldots, h_{3 g-3}}$ associated to $I_{L}(g)$ are polynomials in the three-point functions of fields of weight less than $L$ only. We will call the number of such three-point functions $K_{L}$; it depends on the dimensions of the $L_{0}$-eigenspaces (that can be read off from the torus partition function $Z_{1}$ ), but not on $g$ nor on any other details of the theory. It is clear that there is a non-trivial consistency condition of the above type if

$$
\left.D(g) \mid I_{L}(g)\right) \mid>K_{L},
$$

where $D(g)$ is the number of inequivalent pant decompositions of a genus $g$ surface. Obviously, the left-hand side grows to infinity as $g \rightarrow \infty$, while the right-hand side is independent of $g$. Thus, for sufficiently large $g$, there will be many such consistency conditions. However, if the relevant spaces of states are large (as is, for example, the case for the extremal theories) one has to go to rather large values of $g$ before one obtains non-trivial constraints in this manner.

If we are given a family of higher genus partition functions, then this is all we can say. However, in the context of the extremal ansatz, the natural question is slightly different. Suppose, as for Theorem 4.1, that our putative conformal field theory is of the form (4.1). Can we then construct a family of higher genus amplitudes that not only reproduce the computable coefficients (as in Theorem 4.1), but also allow for a solution of all expansion coefficients in terms of individual structure constants? 
From this point of view, the unknown parameters are the free parameters $\mathcal{P}_{k}(g)$ labelling a genus $g$ modular form of weight $12 k$, as well as the threepoint functions involving three fields from $\mathcal{R}$. The problem is now that the set of coefficients $\mathcal{P}_{k}(g)$ also grows with $g$ and, a priori, these free parameters could allow one to adjust the vacuum amplitudes so that one can always solve for the individual three-point functions (involving fields from $\mathcal{R}$ ). However, this is not the case. As is shown in Appendix D.3, for every $g, \mathcal{P}_{k}(g)$ does not contain any element $\left(h_{1}, \ldots, h_{3 g-3}\right)$ with

$$
h_{1}, \ldots, h_{3 g-3}>L(g) \equiv \frac{k}{5}(g+4) .
$$

[This is the generalization to arbitrary genus of the inequality (2.29).] Let us define, for given $M>0$, the set

$$
I_{L(g), M}(g):=\left\{\left(h_{1}, \ldots, h_{3 g-3}\right): L(g)<h_{1}, \ldots, h_{3 g-3}<L(g)+M\right\}
$$

We want to consider the subsystem of equations that require that the coefficients labelled by $I_{L(g), M}(g)$ in all pant decompositions can be expressed in terms of the three-point functions. Because of (5.11), these equations are unaffected by our ambiguity in the definition of the vacuum amplitudes, i.e., they are independent of the parameters in $\mathcal{P}_{k}(g)$. Furthermore, for large $g$, $L(g)>B$, and all the relevant three-point functions involve mostly fields from $\mathcal{R}$.

With these preparations we can now estimate the number of equations, and the number of unknowns. To leading order in $g$, the number of equations for each pant decomposition goes as

$$
\left|I_{L(g), M}(g)\right| \sim M^{3 g-3}
$$

A rough estimate of the number of different pant decomposition is ${ }^{2}$

$$
D(g) \sim \frac{(6 g-6) !}{2^{3 g-3}(3 g-3) !(3 !)^{2 g-2}(2 g-2) !} \stackrel{g \rightarrow \infty}{\sim}(2 \pi(g-1))^{-1 / 2}\left(\frac{3 g-3}{2 e}\right)^{g-1},
$$

where the right-hand side is a lower bound on the number of double cosets in the space $\left(S_{2}^{3 g-3} \times S_{3 g-3}\right) \backslash S_{6 g-6} /\left(S_{3}^{2 g-2} \times S_{2 g-2}\right)$, with $S_{n}$ the group of permutations of $n$ elements. In fact, each coset corresponds to a decomposition

\footnotetext{
${ }^{2}$ See [34-36] for related asymptotic formulae in graph enumeration problems.
} 
of either a connected or a disconnected surface, but it is easy to see that the contribution of the disconnected surfaces is of order $D(g) / g$ and hence can be ignored in our leading order approximation. Thus, the number of equations in the subsystem relative to $I_{L(g), M}(g)$ is

$$
\text { \# equations } \sim g^{-1 / 2}\left(\frac{M^{3}(3 g-3)}{2 e}\right)^{g-1} .
$$

The number of unknowns is roughly speaking the number of structure constants up to weight $L(g)+M$, so that ${ }^{3}$

$$
\text { \# unknowns } \sim\left(\sum_{h \leq L(g)+M} \operatorname{dim} \mathcal{H}_{h}\right)^{3}=\left(\sum_{h \leq L(g)+M} n_{h}\right)^{3} .
$$

Here, $n_{h}$ are the coefficients in the $q$-expansion of the modular invariant torus partition function

$$
\frac{Z_{1}(q)}{\Delta^{k}}=\sum_{h=0}^{\infty} n_{h} q^{h-k}, \quad q=\mathrm{e}^{2 \pi \mathrm{i} \tau}
$$

where $\Delta=q+\mathcal{O}\left(q^{2}\right)$ has modular weight 12 . For large $h$ the Cardy formula

$$
n_{l+k} \sim \frac{k^{1 / 4}}{\sqrt{2}} l^{-3 / 4} \mathrm{e}^{4 \pi \sqrt{k l}}, \quad l \gg 0
$$

applies (see Appendix D for details). Note that, at leading order in $h \rightarrow \infty$, $n_{h}$ only depends on the modular weight $12 k$ of $Z_{1}$ and on the normalization $n_{0}=1$. Thus, taking just the leading contribution $h=L(g)+M$ in the sum (5.16), the requirement that the system of equations is over-determined is

$$
g^{-1 / 2}\left(\frac{M^{3}(3 g-3)}{2 e}\right)^{g-1} \gtrsim(L(g)+M-k)^{-9 / 4} \mathrm{e}^{12 \pi \sqrt{k(L(g)+M-k)}}
$$

up to some factor independent of $g$ and $M$. Now, if we take $M>0$ fixed and $g$ large enough so that $L(g) \sim k g / 5 \gg M-k$, Equation (5.19) becomes

$$
g^{-1 / 2}\left(\frac{M^{3}(3 g-3)}{2 e}\right)^{g-1} \gtrsim g^{-9 / 4}\left(\mathrm{e}^{\frac{12 \pi k}{\sqrt{5}}}\right)^{\sqrt{g}}
$$

\footnotetext{
${ }^{3}$ Actually, we are overestimating the number of unknowns, because we are also including the structure constants of $\mathcal{A}$, but this does not affect the following reasoning.
} 
which is satisfied for sufficiently large $g$. Thus there will be many obstructions to the construction of a family of modular invariant genus $g$ partition functions if we demand that their coefficients can be expressed in terms of contracted structure constants.

To see how this estimate works in practice, let us consider the example of the extremal ansatz at $k=2$. The estimate (5.20) indicates that, for $M<5$, the first constraints on the structure constants should arise for $20 \lesssim g \lesssim 40$. A more precise computation, using (5.14) and (5.16) with the correct values for $n_{h}$, shows that consistency of the partition function at genus $g=23$ puts non-trivial constraints on the structure constants up to conformal weight $h=L(g)+M=17$. In practice, however, amplitudes of such a high genus are unfortunately not accessible.

\section{Conclusions}

In this paper we have analysed the structure of the genus $g=2$ vacuum amplitudes of chiral self-dual conformal field theories. In particular, we have shown that the existence of a modular invariant $g=2$ partition function implies infinitely many relations among the structure constants of the theory. All of these relations are a consequence of the associativity of the OPE, as well as the modular covariance of the torus one-point amplitudes (Theorem 3.1). This was to be expected from the point of view of the Moore and Seiberg analysis [1].

We have applied these techniques to the extremal ansatz, and we have shown that a (seemingly) consistent genus $g=2$ vacuum amplitude exists for the extremal theories at $c=24 k$ for all $k$ (Theorem 4.1). However, we have also shown that this does not check any consistency conditions of the extremal ansatz beyond what can already be tested by analysing the modular covariance properties of the ansatz at genus one.

Finally, we have shown that a family of modular invariant genus $g$ partition functions can only define a consistent conformal field theory if one can actually reconstruct the individual structure constants whose contracted expressions reproduce the expansion coefficients of the vacuum amplitudes. As we have demonstrated with two examples (see Section 5.1), this is a nontrivial consistency condition that does not seem to be implied by modular invariance. The rough counting argument of Section 5.2 suggests that it will also lead to a stringent constraint for the extremal ansatz. However, as is also clear from that analysis, this constraint will only become interesting at rather large genera - and hence is unfortunately, technically fairly out 
of reach. Thus to prove or disprove the existence of the extremal ansatz by studying higher genus partition functions seems to be as difficult as the brute force approach of analysing the Jacobi identities of the fields at large conformal weight.

\section{Acknowledgments}

We thank Terry Gannon for a useful conversation and subsequent correspondence. C.A.K. thanks the Pauli Center for support during his visit to ETH. The research of M.R.G. is partially supported by the Swiss National Science Foundation, while the research of R.V. is supported by an INFN Fellowship.

\section{Appendix A. Riemann surfaces, Schottky groups and modular forms}

\section{A.1. Riemann period matrices and modular forms}

In order to analyse the modular properties of partition functions, it is useful to define the period matrix of a Riemann surface. Let $\Sigma$ be a compact Riemann surface of genus $g>0$. Let us define a basis of the first homology group $H_{1}(\Sigma, \mathbb{Z})\left\{\alpha_{1}, \ldots, \alpha_{g}, \beta_{1}, \ldots, \beta_{g}\right\}$, with symplectic intersection matrix

$$
\#\left(\alpha_{i}, \alpha_{j}\right)=0=\#\left(\beta_{i}, \beta_{j}\right), \quad \#\left(\alpha_{i}, \beta_{j}\right)=\delta_{i j}, \quad i, j=1, \ldots, g
$$

This condition determines the basis up to a symplectic transformation

$$
\left(\begin{array}{c}
\alpha \\
\beta
\end{array}\right) \longmapsto\left(\begin{array}{c}
\tilde{\alpha} \\
\tilde{\beta}
\end{array}\right):=\left(\begin{array}{ll}
D & C \\
B & A
\end{array}\right)\left(\begin{array}{l}
\alpha \\
\beta
\end{array}\right), \quad\left(\begin{array}{ll}
A & B \\
C & D
\end{array}\right) \in \operatorname{Sp}(2 g, \mathbb{Z})
$$

where $\alpha$ and $\beta$ are $g$-dimensional vectors, and $A, B, C, D$ are $g \times g$ matrices. The choice of such a basis uniquely determines a basis $\left\{\omega_{1}, \ldots, \omega_{g}\right\}$ of holomorphic 1-differentials normalized with respect to the $\alpha$-cycles

$$
\oint_{\alpha_{i}} \omega_{j}=\delta_{i j}, \quad i, j=1, \ldots, g .
$$

The Riemann period matrix of $\Sigma$ is then defined by

$$
\Omega_{i j}=\oint_{\beta_{i}} \omega_{j}
$$


332 Matthias R. Gaberdiel, Christoph A. Keller and Roberto Volpato

and it has the properties

$$
\Omega_{i j}=\Omega_{j i}, \quad \operatorname{Im} \Omega>0 .
$$

Obviously, the basis $\left\{\omega_{1}, \ldots, \omega_{g}\right\}$, and the Riemann period matrix depends on the choice of the symplectic basis of $H_{1}(\Sigma, \mathbb{Z})$; under the action (A.2) of the symplectic group, the holomorphic 1-differentials and the period matrix transform as

$$
\begin{aligned}
\left(\omega_{1}, \ldots, \omega_{g}\right) & \longmapsto\left(\tilde{\omega}_{1}, \ldots, \tilde{\omega}_{g}\right)=\left(\omega_{1}, \ldots, \omega_{g}\right)(C \Omega+D)^{-1}, \\
\Omega & \longmapsto \tilde{\Omega}=(A \Omega+B)(C \Omega+D)^{-1} .
\end{aligned}
$$

Let us define the Siegel upper half-space as the space of $g \times g$ symmetric complex matrices with positive-definite imaginary part,

$$
\mathfrak{H}_{g}=\left\{Z \in M_{g}(\mathbb{C}) \mid Z_{i j}=Z_{j i}, \operatorname{Im} Z>0\right\} .
$$

The locus $\mathcal{J}_{g} \subseteq \mathfrak{H}_{g}$ of all the period matrices of genus $g$ Riemann surfaces is dense in $\mathfrak{H}_{g}$ for $g \leq 3$, whereas for $g>3$ it is a $(3 g-3)$-dimensional subspace of $\mathfrak{H}_{g}$. The quotient $\mathcal{J}_{g} / \operatorname{Sp}(2 g, \mathbb{Z})$ is isomorphic to $\mathcal{M}_{g}$; in particular, the Riemann period matrices of two different Riemann surfaces lie in different $\operatorname{Sp}(2 g, \mathbb{Z})$-orbits in $\mathcal{J}_{g}$.

\section{A.2. Modular forms of degree 1 and 2}

A (Siegel) modular form $f$ of degree $g$ and weight $k$ is a holomorphic function on $\mathfrak{H}_{g}$ such that

$$
\begin{gathered}
f\left((A Z+B)(C Z+D)^{-1}\right)=\operatorname{det}(C Z+D)^{k} f(Z), \\
M=\left(\begin{array}{ll}
A & B \\
C & D
\end{array}\right) \in \operatorname{Sp}(2 g, \mathbb{Z}) .
\end{gathered}
$$

For $g=1$ we also require that $f$ is holomorphic at the cusps; a cusp is a fixpoint $p \in \mathbb{R} \cup\{\infty\}$ under the action of some $M \in \operatorname{Sp}(2, \mathbb{Z}) \cong S L(2, \mathbb{Z})$ with $\operatorname{Tr}(M)= \pm 2$ (a parabolic element). An analogous condition is automatically satisfied for $g>1$.

The space of modular forms of degree 1 is generated by the Eisenstein series $G_{4}$ and $G_{6}$ defined by

$$
G_{k}(q)=1+\frac{2}{\zeta(1-k)} \sum_{n=1}^{\infty} \sigma_{k-1}(n) q^{n}
$$


where $\zeta$ is the Riemann $\zeta$-function, and

$$
\sigma_{k}(n)=\sum_{d \mid n} d^{k} .
$$

All Siegel modular forms of degree 2 can be written in terms of Eisenstein series

$$
E_{k}:=\mathcal{N}_{k} \sum_{C, D} \operatorname{det}(C \Omega+D)^{-k},
$$

where $\mathcal{N}_{k}$ is a normalization constant, and $C$ and $D$ are $2 \times 2$ integral matrices such that $\left(\begin{array}{ll}A & B \\ C & D\end{array}\right) \in \mathrm{Sp}(4, \mathbb{Z})$. The sum is over all the inequivalent pairs under left multiplication by elements of $\mathrm{GL}(2, \mathbb{Z})[37,38]$. The Eisenstein series admits a Fourier expansion ([37, pp. 17-18])

$$
E_{k}=\sum_{n, m=0}^{\infty} \sum_{\substack{r \in \mathbb{Z} \\ r^{2} \leq 4 n m}} a_{k}(n, m, r) q_{11}^{n} q_{22}^{m} q_{12}^{r}
$$

where

$$
q_{i j}:=\mathrm{e}^{2 \pi \mathrm{i} \Omega_{i j}},
$$

and

$$
\text { (A.14) } a_{k}(n, m, r)=\frac{2}{\zeta(3-2 k) \zeta(1-k)} \sum_{d \mid(n, m, r)} d^{k-1} H\left(k-1, \frac{4 n m-r^{2}}{d^{2}}\right) \text {. }
$$

Here, $H$ is Cohen's function (see [39, pp. 21-22] for a definition) and the normalization is chosen so that $a_{k}(0,0,0)=1$.

The ring of (even) Siegel modular forms of degree two is freely generated by

$$
\begin{aligned}
\psi_{4} & =E_{4}, \quad \chi_{10}=\frac{43867}{2^{12} \cdot 3^{5} \cdot 5^{2} \cdot 7 \cdot 53}\left(E_{10}-E_{4} E_{6}\right), \\
(\text { A.15 }) \quad \psi_{6} & =E_{6}, \quad \chi_{12}=\frac{131 \cdot 593}{2^{13} \cdot 3^{7} \cdot 5^{3} \cdot 7^{2} \cdot 337}\left(21^{2} E_{4}^{3}+250 E_{6}^{2}-691 E_{12}\right) .
\end{aligned}
$$

\section{A.3. Schottky parameters}

The Schottky uniformization describes a general non-singular Riemann surface as the quotient of the Riemann sphere $\hat{\mathbb{C}}=\mathbb{C} \cup\{\infty\}$ by a suitable subgroup of $\operatorname{PSL}(2, \mathbb{C})$. Geometrically, a surface $\Sigma$ of genus $g>0$ is obtained 
334 Matthias R. Gaberdiel, Christoph A. Keller and Roberto Volpato

by cutting $2 g$ disks from $\hat{\mathbb{C}}$, bounded by non-intersecting circles $C_{1}, \ldots, C_{g}$, $C_{-1}, \ldots, C_{-g}$, and then by identifying each circle $C_{i}$ with the circle $C_{-i}$ via a suitable fractional linear transformation $\gamma_{i} \in \operatorname{PSL}(2, \mathbb{C})$ such that

$$
\gamma_{i} C_{-i}=C_{i},
$$

for all $i=1, \ldots, g$ (see Figure A.1). The discrete subgroup $\Gamma$ with distinct free generators $\gamma_{1}, \ldots, \gamma_{g}$ is called a marked Schottky group. Each $\gamma \in \Gamma$ is characterized by two distinct points $a_{\gamma}, r_{\gamma} \in \hat{\mathbb{C}}$ (called the attractive and repelling fixed point, respectively) and a complex number $p_{\gamma} \in \mathbb{C}$ (the multiplier), with $0<\left|p_{\gamma}\right|<1$, such that

$$
\frac{\gamma(z)-a_{\gamma}}{\gamma(z)-r_{\gamma}}=p_{\gamma} \frac{z-a_{\gamma}}{z-r_{\gamma}} \quad \text { for all } z \in \hat{\mathbb{C}} .
$$

The Riemann surface $\Sigma$ can be obtained as the quotient of $\hat{\mathbb{C}}$ by $\Gamma$, and every non-singular closed surface can be obtained in this way. By a $\operatorname{PSL}(2, \mathbb{C})$ conjugation, one can fix

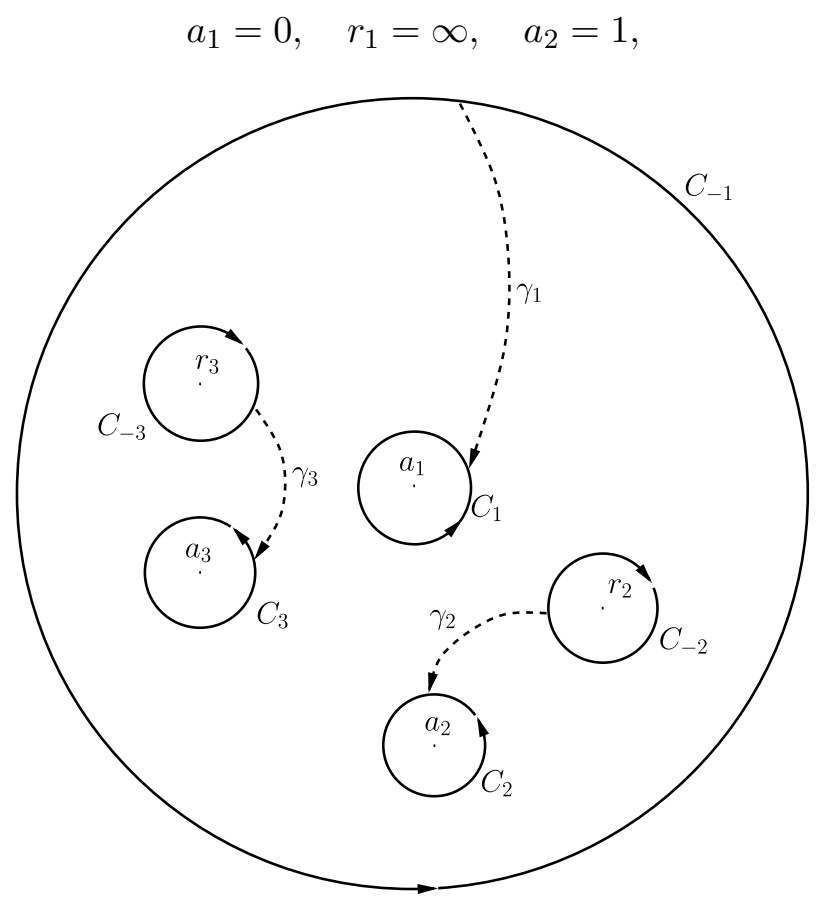

Figure A.1: A fundamental domain for a Schottky group. Each generator $\gamma_{i}, i=1, \ldots, g$, with fixed points $a_{i}, r_{i}$, maps the circle $C_{-i}$ to the circle $C_{i}$. 
where we set

$$
a_{i}:=a_{\gamma_{i}}, \quad r_{i}:=r_{\gamma_{i}}, \quad p_{i}:=p_{\gamma_{i}} .
$$

When (A.18) holds, the marked Schottky group is called normalized. The space $\mathfrak{S}_{g}$ of normalized marked Schottky groups of genus $g$ is parametrized by $3 g-3$ coordinates

$$
\left\{p_{1}, \ldots, p_{g}, a_{3}, \ldots, a_{g}, r_{2}, \ldots, r_{g}\right\}
$$

so that $\mathfrak{S}_{g}$ can be identified with a domain in $\mathbb{C}^{g} \times \hat{\mathbb{C}}^{2 g-3}$. More precisely, the condition that there are $2 g$ non-intersecting circles $C_{ \pm i}, i=1, \ldots, g$, satisfying (A.16) implies

$$
\begin{gathered}
\text { (A.21) }\left|p_{i}\right|<\min \left\{\left|\frac{\left(x_{j}-x_{i}\right)\left(x_{k}-x_{-i}\right)}{\left(x_{k}-x_{i}\right)\left(x_{j}-x_{-i}\right)}\right|: j, k= \pm 1, \ldots, \pm g, j, k \neq \pm i\right\} \\
i=1, \ldots, g
\end{gathered}
$$

where $x_{i}:=a_{i}, x_{-i}:=r_{i}, i=1, \ldots, g$. The space $\mathfrak{S}_{g}$ is a finite covering of the moduli space $\mathcal{M}_{g}$ of Riemann surfaces. Given a Riemann surface $\Sigma$ uniformized by the Schottky group $\Gamma$, we can take the homology classes of $C_{1}, \ldots, C_{g}$ as the generators $\alpha_{1}, \ldots, \alpha_{g}$ in the symplectic basis of $H_{1}(\Sigma, \mathbb{Z})$ (see Appendix A.1). Thus, the choice of the group $\Gamma$ uniformizing $\Sigma$ also gives a canonical choice for the basis $\omega_{1}, \ldots, \omega_{g}$ of holomorphic 1-differentials satisfying (A.3). The Riemann period matrix $\Omega$ depends also on the choice of the $\beta$-cycles. However, the multiplicative periods $\exp \left(2 \pi i \Omega_{i j}\right)$ are welldefined functions of the parameters (A.20). More precisely, one can prove that

$$
\begin{aligned}
& q_{i i}=\mathrm{e}^{2 \pi \mathrm{i} \Omega_{i i}}=p_{i} \prod_{\gamma \in\left\langle\gamma_{i}\right\rangle \backslash \Gamma /\left\langle\gamma_{i}\right\rangle} \frac{\left(a_{i}-\gamma\left(a_{i}\right)\right)\left(r_{i}-\gamma\left(r_{i}\right)\right)}{\left(a_{i}-\gamma\left(r_{i}\right)\right)\left(r_{i}-\gamma\left(a_{i}\right)\right)} \\
& q_{i j}=\mathrm{e}^{2 \pi \mathrm{i} \Omega_{i j}}=\prod_{\gamma \in\left\langle\gamma_{i}\right\rangle \backslash \Gamma /\left\langle\gamma_{j}\right\rangle} \frac{\left(a_{i}-\gamma\left(a_{j}\right)\right)\left(r_{i}-\gamma\left(r_{j}\right)\right)}{\left(a_{i}-\gamma\left(r_{j}\right)\right)\left(r_{i}-\gamma\left(a_{j}\right)\right)}, \quad i \neq j,
\end{aligned}
$$

where $\left\langle\gamma_{i}\right\rangle$ is the group generated by $\gamma_{i}, i=1, \ldots, g$. Thus, for each element in $\left\langle\gamma_{i}\right\rangle \backslash \Gamma /\left\langle\gamma_{j}\right\rangle$, we can choose a representative with reduced word $\gamma_{k_{1}} \cdots \gamma_{k_{n}}$, for $k_{1} \neq i$ and $k_{n} \neq j$. 
336 Matthias R. Gaberdiel, Christoph A. Keller and Roberto Volpato

In the case of $g=2$, the Schottky space $\mathfrak{S}_{2}$ is parametrized by

$$
p_{1}, \quad p_{2}, \quad r_{2} \equiv x
$$

and the condition (A.21) gives the identification

$$
\begin{gathered}
\mathfrak{S}_{2} \equiv\left\{\left(p_{1}, p_{2}, x\right) \in \mathbb{C} \times \mathbb{C} \times \hat{\mathbb{C}}: x \neq 0,1, \infty,\right. \\
\left.0<\left|p_{i}\right|<\min \left(|x|, \frac{1}{|x|}\right), i=1,2\right\} .
\end{gathered}
$$

The power series expansions of the multiplicative periods are

$$
\begin{aligned}
& q_{11}=\mathrm{e}^{2 \pi \mathrm{i} \Omega_{11}}=p_{1} \sum_{n, m=0}^{\infty} \sum_{r=-n-m}^{n+m} c(n, m,|r|) p_{1}^{n} p_{2}^{m} x^{r}, \\
& q_{22}=\mathrm{e}^{2 \pi \mathrm{i} \Omega_{22}}=p_{2} \sum_{n, m=0}^{\infty} \sum_{r=-n-m}^{n+m} c(m, n,|r|) p_{1}^{n} p_{2}^{m} x^{r}, \\
& q_{12}=\mathrm{e}^{2 \pi \mathrm{i} \Omega_{12}}=x+x \sum_{n, m=1}^{\infty} \sum_{r=-n-m}^{n+m} d(n, m, r) p_{1}^{n} p_{2}^{m} x^{r} .
\end{aligned}
$$

The coefficients $c(m, n, r)$ for $n \leq 6$ and $m \leq 7$ are listed in tables 1 and 2, while $d(m, n, r)=d(n, m, r)$ for $m, n \leq 7$ are listed in tables 3 and 4 .

Table 1: Coefficients $c(n, m,|r|)$ in the expansions (A.26) of $q_{11}$ and (A.27) of $q_{22}$ up to $p_{1}^{7} p_{2}^{7}$.

\begin{tabular}{lcrrrrrrr}
\hline$c(m, n,|r|)$ & \multicolumn{10}{c}{$m, n$} \\
\hline$|r|$ & 0,0 & 0,1 & 0,2 & 0,3 & 0,4 & 0,5 & 0,6 & 0,7 \\
\hline 0 & 1 & -4 & 6 & 0 & -8 & 0 & 10 & 0 \\
1 & 0 & 2 & -6 & 4 & 4 & 0 & -10 & 0 \\
2 & 0 & 0 & 3 & -8 & 5 & 0 & 5 & 0 \\
3 & 0 & 0 & 0 & 4 & -10 & 6 & 0 & 0 \\
4 & 0 & 0 & 0 & 0 & 5 & -12 & 7 & 0 \\
5 & 0 & 0 & 0 & 0 & 0 & 6 & -14 & 8 \\
6 & 0 & 0 & 0 & 0 & 0 & 0 & 7 & -16 \\
7 & 0 & 0 & 0 & 0 & 0 & 0 & 0 & 8 \\
\hline & & & & & & & & Continued
\end{tabular}


Genus two partition functions of chiral conformal field theories 337

Table 1: Continued

\begin{tabular}{|c|c|c|c|c|c|c|c|c|}
\hline$\overline{c(m, n,|r|)}$ & & & & & $m, n$ & & & \\
\hline$\overline{|r|}$ & 1,0 & 1,1 & 1,2 & 1,3 & 1,4 & 1,5 & 1,6 & 1,7 \\
\hline$\overline{0}$ & 0 & 0 & 8 & -32 & 56 & -96 & 224 & -384 \\
\hline 1 & 0 & 0 & -2 & 20 & -58 & 100 & -186 & 332 \\
\hline 2 & 0 & 0 & -4 & 8 & 28 & -96 & 132 & -216 \\
\hline 3 & 0 & 0 & 2 & -20 & 38 & 20 & -102 & 136 \\
\hline 4 & 0 & 0 & 0 & 8 & -56 & 104 & -24 & -96 \\
\hline 5 & 0 & 0 & 0 & 0 & 20 & -120 & 218 & -104 \\
\hline 6 & 0 & 0 & 0 & 0 & 0 & 40 & -220 & 392 \\
\hline 7 & 0 & 0 & 0 & 0 & 0 & 0 & 70 & -364 \\
\hline 8 & 0 & 0 & 0 & 0 & 0 & 0 & 0 & 112 \\
\hline$c(m, n,|r|)$ & & & & & $m, n$ & & & \\
\hline$|r|$ & 2,0 & 2,1 & 2,2 & 2,3 & 2,4 & 2,5 & 2,6 & 2,7 \\
\hline 0 & 0 & 0 & 64 & -280 & 436 & -936 & 3186 & -5712 \\
\hline 1 & 0 & 0 & -50 & 240 & -422 & 864 & -2696 & 4868 \\
\hline 2 & 0 & 0 & 28 & -168 & 385 & -704 & 1710 & -3072 \\
\hline 3 & 0 & 0 & -14 & 122 & -392 & 686 & -1040 & 1568 \\
\hline 4 & 0 & 0 & 4 & -76 & 410 & -996 & 1304 & -1120 \\
\hline 5 & 0 & 0 & 0 & 22 & -274 & 1194 & -2468 & 2608 \\
\hline 6 & 0 & 0 & 0 & 0 & 75 & -776 & 3002 & -5648 \\
\hline 7 & 0 & 0 & 0 & 0 & 0 & 200 & -1860 & 6672 \\
\hline 8 & 0 & 0 & 0 & 0 & 0 & 0 & 455 & -3944 \\
\hline 9 & 0 & 0 & 0 & 0 & 0 & 0 & 0 & 924 \\
\hline$c(m, n,|r|)$ & & & & & $m, n$ & & & \\
\hline$|r|$ & 3,0 & 3,1 & 3,2 & 3,3 & 3,4 & 3,5 & 3,6 & 3,7 \\
\hline$\overline{0}$ & 0 & 0 & 80 & -464 & 2480 & -8832 & 24,848 & $-66,544$ \\
\hline 1 & 0 & 0 & -44 & 344 & -1996 & 7828 & $-22,954$ & 60,440 \\
\hline 2 & 0 & 0 & -16 & -76 & 860 & -5048 & 17,988 & $-47,496$ \\
\hline 3 & 0 & 0 & 38 & -172 & 354 & 1220 & $-10,670$ & 34,724 \\
\hline 4 & 0 & 0 & -24 & 264 & -1256 & 2824 & 576 & $-19,848$ \\
\hline 5 & 0 & 0 & 6 & -172 & 1442 & -5876 & 11,846 & -4988 \\
\hline 6 & 0 & 0 & 0 & 44 & -844 & 5944 & $-21,188$ & 39,616 \\
\hline 7 & 0 & 0 & 0 & 0 & 200 & -3172 & 19,762 & $-64,060$ \\
\hline 8 & 0 & 0 & 0 & 0 & 0 & 696 & -9800 & 55,904 \\
\hline 9 & 0 & 0 & 0 & 0 & 0 & 0 & 2016 & $-26,116$ \\
\hline 10 & 0 & 0 & 0 & 0 & 0 & 0 & 0 & 5096 \\
\hline
\end{tabular}


338 Matthias R. Gaberdiel, Christoph A. Keller and Roberto Volpato

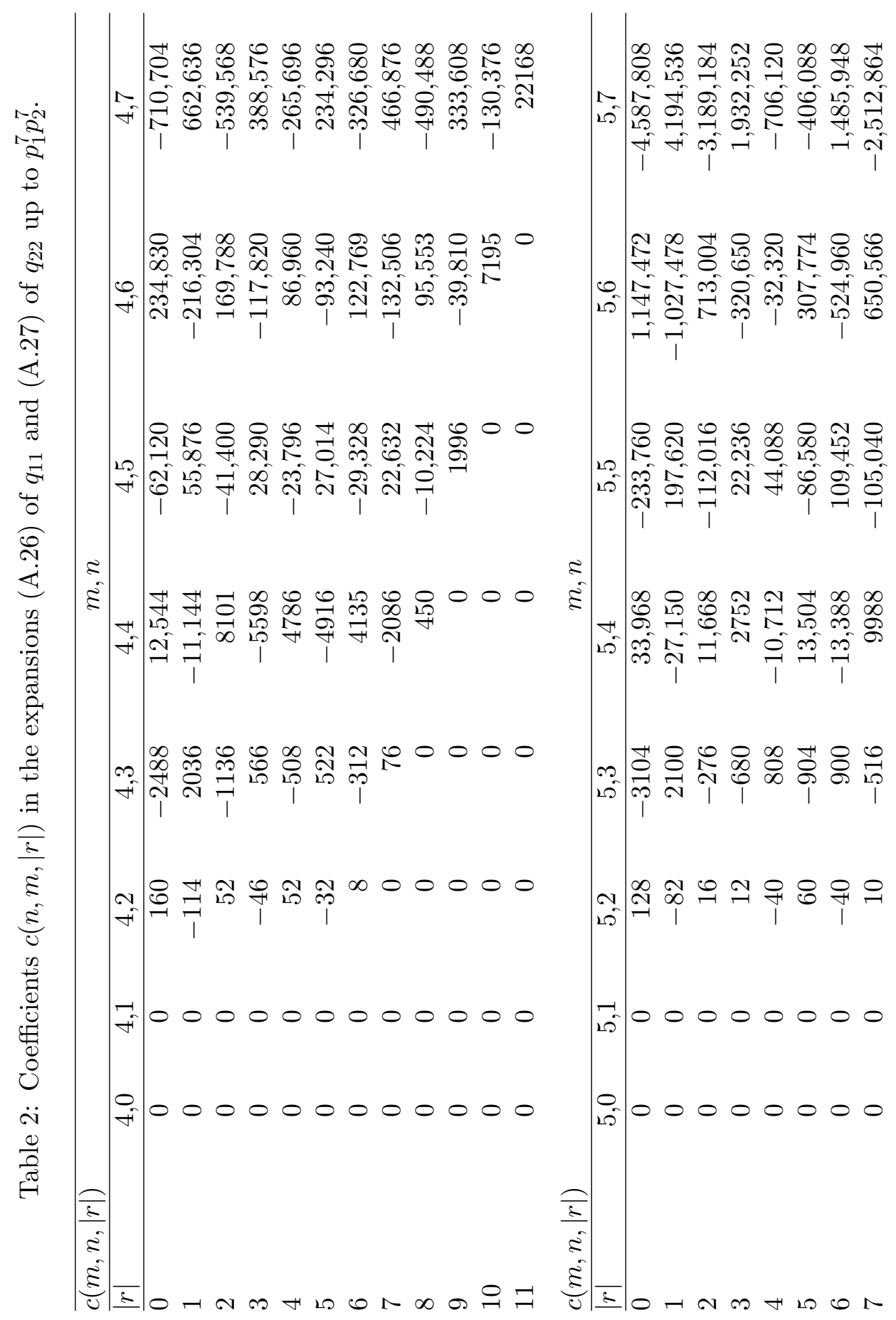


Genus two partition functions of chiral conformal field theories 339

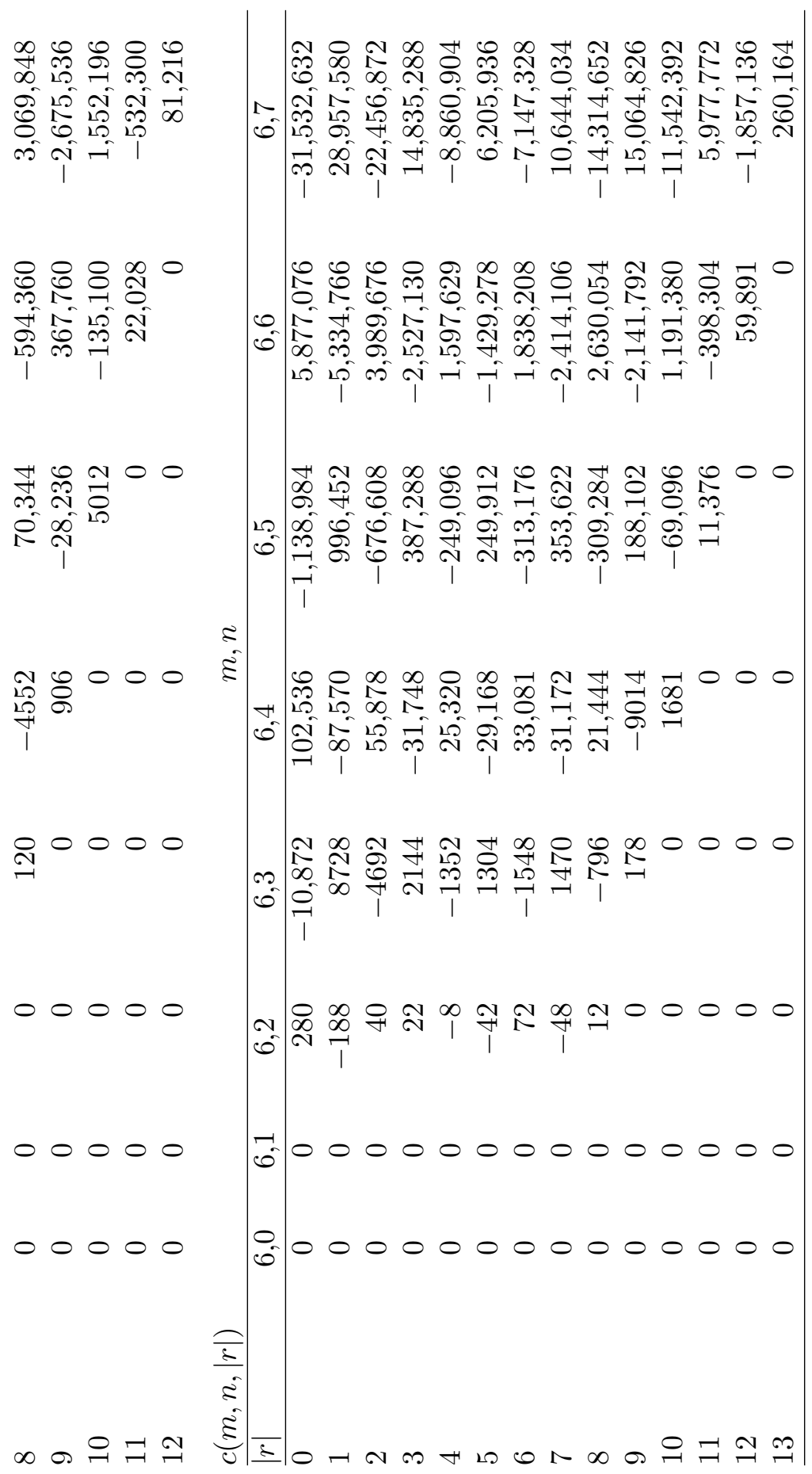


340 Matthias R. Gaberdiel, Christoph A. Keller and Roberto Volpato

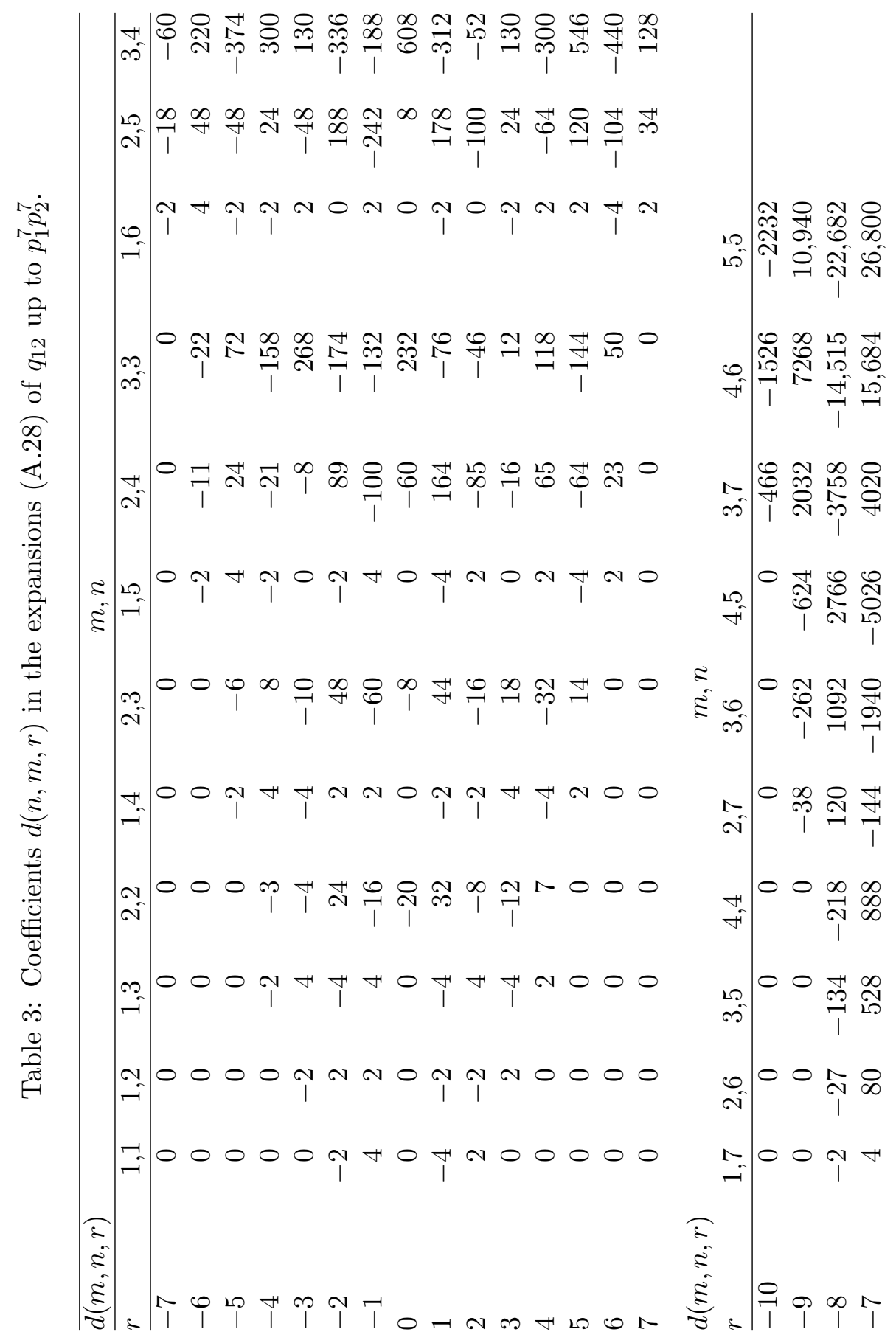


Genus two partition functions of chiral conformal field theories 341

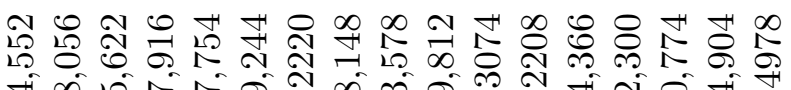

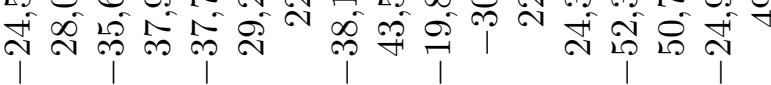

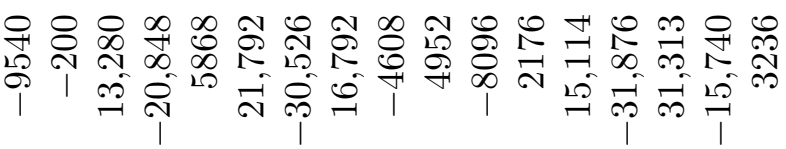

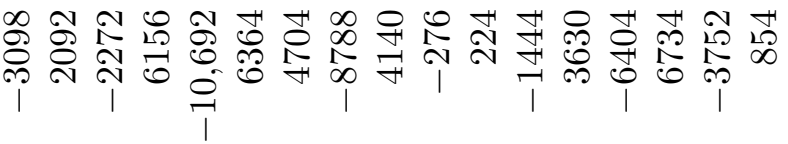

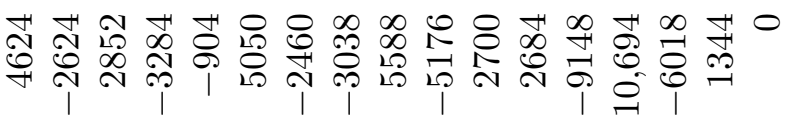

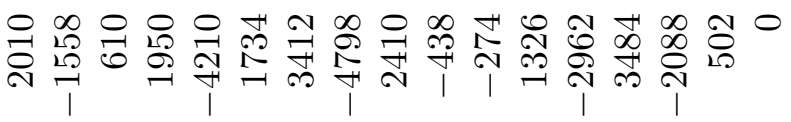

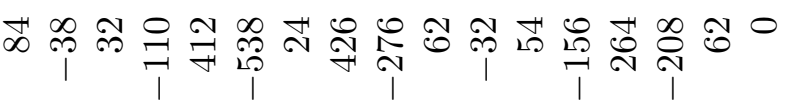

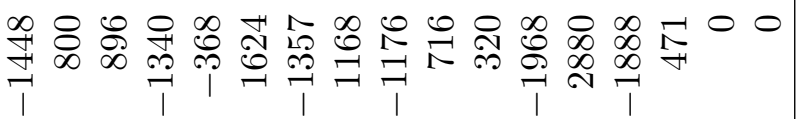

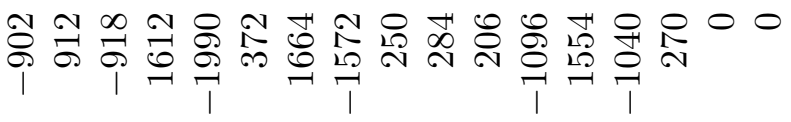

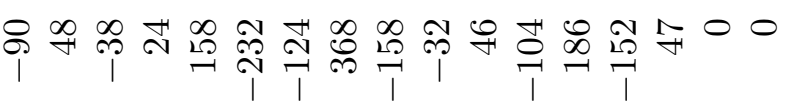
io $\begin{array}{llllll}1 & 1 \\ 0 & 1 & 1 & 1\end{array}$ 
342 Matthias R. Gaberdiel, Christoph A. Keller and Roberto Volpato

Table 4: Coefficients $d(n, m, r)$ in the expansions (A.28) of $q_{12}$ up to $p_{1}^{7} p_{2}^{7}$.

\begin{tabular}{|c|c|c|c|c|c|c|}
\hline$\overline{d(m, n, r)}$ & & & & $m, n$ & & \\
\hline $\mathrm{r}$ & 4,7 & 5,6 & 5,7 & 6,6 & 6,7 & 7,7 \\
\hline$\overline{-14}$ & 0 & 0 & 0 & 0 & 0 & $-279,508$ \\
\hline-13 & 0 & 0 & 0 & 0 & $-75,924$ & $1,927,676$ \\
\hline-12 & 0 & 0 & $-17,630$ & $-24,228$ & 481,740 & $-5,998,022$ \\
\hline-11 & -3330 & -6694 & 100,956 & 142,216 & $-1,364,286$ & $11,228,868$ \\
\hline-10 & 16,836 & 35,728 & $-253,434$ & $-368,166$ & $2,304,584$ & $-14,286,298$ \\
\hline-9 & $-36,242$ & $-82,456$ & 373,560 & 563,140 & $-2,636,708$ & $13,345,304$ \\
\hline-8 & 43,556 & 110,530 & $-375,412$ & $-581,504$ & $2,209,596$ & $-10,053,096$ \\
\hline-7 & $-31,916$ & $-102,420$ & 303,168 & 424,564 & $-1,441,188$ & $7,731,048$ \\
\hline-6 & 13,316 & 77,064 & $-265,734$ & $-156,462$ & 840,784 & $-8,536,940$ \\
\hline-5 & -5756 & $-46,470$ & 334,836 & $-162,908$ & $-670,488$ & $11,904,316$ \\
\hline-4 & 25,424 & 16,218 & $-467,020$ & 433,181 & 841,880 & $-14,909,588$ \\
\hline-3 & $-53,870$ & 3862 & 516,756 & $-506,252$ & $-1,008,406$ & $14,283,736$ \\
\hline-2 & 34,092 & $-27,582$ & $-410,996$ & 240,120 & 785,932 & $-8,781,634$ \\
\hline-1 & 32,538 & 48,822 & 184,292 & 293,204 & $-164,092$ & 221,276 \\
\hline 0 & $-62,216$ & $-14,228$ & 127,836 & $-707,758$ & $-289,064$ & $7,537,956$ \\
\hline 1 & 21,398 & $-63,242$ & $-399,996$ & 725,940 & 122,532 & $-10,976,964$ \\
\hline 2 & 30,028 & 94,466 & 436,396 & $-500,264$ & 368,740 & $9,267,180$ \\
\hline 3 & $-45,058$ & $-65,554$ & $-247,420$ & 288,796 & $-681,688$ & $-4,644,648$ \\
\hline 4 & 35,136 & 41,606 & 31,570 & $-175,530$ & 734,824 & 164,070 \\
\hline 5 & $-18,408$ & $-31,580$ & 81,704 & 137,264 & $-692,102$ & $2,588,888$ \\
\hline 6 & $-10,816$ & $-25,534$ & $-34,490$ & $-23,790$ & 468,580 & $-3,291,630$ \\
\hline 7 & 56,288 & 143,206 & $-232,388$ & $-378,460$ & 511,608 & 850,620 \\
\hline 8 & $-89,580$ & $-225,998$ & 614,472 & 953,710 & $-2,611,364$ & $6,910,004$ \\
\hline 9 & 77,454 & 187,450 & $-784,572$ & $-1,191,972$ & $4,740,264$ & $-18,914,964$ \\
\hline 10 & $-35,776$ & $-82,270$ & 574,978 & 854,578 & $-4,995,180$ & $27,421,428$ \\
\hline 11 & 6902 & 15,076 & $-230,896$ & $-335,532$ & $3,183,372$ & $-24,917,120$ \\
\hline 12 & 0 & 0 & 39,464 & 56,113 & $-1,141,052$ & $14,172,292$ \\
\hline 13 & 0 & 0 & 0 & 0 & 177,106 & $-4,628,036$ \\
\hline 14 & 0 & 0 & 0 & 0 & 0 & 663,786 \\
\hline
\end{tabular}

\section{Appendix B. Partition functions}

The genus $g$ partition function $Z_{g}$ of a chiral conformal field theory is defined, in physics, as the vacuum expectation value of the theory on the Riemann 
surface of genus $g$. Due to the conformal anomaly, the partition function, however, depends not only on the complex structure on the surface, but also on the specific choice of coordinates on it. As a consequence, $Z_{g}$ cannot be defined as a globally holomorphic function on $\mathcal{M}_{g}$, but rather only as a holomorphic section on a suitable line bundle on $\mathcal{M}_{g}$. (Alternatively, we may define $Z_{g}$ as a holomorphic function on some covering space of $\mathcal{M}_{g}$, such as the space of Riemann period matrices $\mathcal{J}_{g} \subseteq \mathfrak{H}_{g}$ or the Schottky space $\mathfrak{S}_{g}$.) More precisely, the partition function of a chiral conformal field theory with central charge $c$ can be represented as a holomorphic section ${ }^{4}$ of $\mathcal{L}^{\otimes c / 2}[7,40]$, where $\mathcal{L}$ is the Hodge bundle.

The Hodge bundle $\mathcal{L}$ can be described as follows. Consider the vector bundle $\Lambda_{g}$ of rank $g$ on $\mathcal{M}_{g}$, whose fibre at the point corresponding to the Riemann surface $\Sigma$ is the $g$-dimensional vector space of holomorphic 1-differentials on $\Sigma$. As shown in Appendix A.1, the choice of a symplectic basis for the first homology group $H_{1}(\Sigma, \mathbb{Z})$ determines a basis $\left\{\omega_{1}, \ldots, \omega_{g}\right\}$ of holomorphic 1-differentials on $\Sigma$, and hence a basis of local sections on $\Lambda_{g}$, which we also denote by $\omega_{1}, \ldots, \omega_{g}$. The line bundle $\mathcal{L}$ is then defined as the $g$ th exterior product of $\Lambda_{g}$, and given a choice of a basis for $H_{1}(\Sigma, \mathbb{Z})$, $\omega_{1} \wedge \ldots \wedge \omega_{g}$ defines a local holomorphic section in $\mathcal{L}$. Under a symplectic transformation (A.2) the corresponding local section of $\mathcal{L}$ transforms as

$$
\begin{gathered}
\omega_{1} \wedge \ldots \wedge \omega_{g} \longmapsto \operatorname{det}(C \Omega+D)^{-1}\left(\omega_{1} \wedge \ldots \wedge \omega_{g}\right) \\
\text { where }\left(\begin{array}{ll}
A & B \\
C & D
\end{array}\right) \in \operatorname{Sp}(2 g, \mathbb{Z}) .
\end{gathered}
$$

The partition function $Z_{g}$ of a meromorphic CFT is a global holomorphic section of $\mathcal{L}^{c / 2}$, so that it can be written locally as

$$
Z_{g}=W_{g}(\Omega)\left(\omega_{1} \wedge \ldots \wedge \omega_{g}\right)^{c / 2}
$$

where $W_{g}$ is a holomorphic function on the space $\mathcal{J}_{g} \subset \mathfrak{H}_{g}$ of period matrices of Riemann surfaces. Since the section cannot depend on the choice of the

\footnotetext{
${ }^{4}$ We observe that $\mathcal{L}^{c / 2}$ is a well-defined line bundle on $\mathcal{M}_{g}$ only if $c$ is multiple of 4 , which is the case for meromorphic conformal field theories. In the other cases, it can only be defined as a projective line bundle $[7,40]$.
} 
local trivialization, $W_{g}$ must transform as a modular form of weight $c / 2$

$$
W_{g}\left((A \Omega+B)(C \Omega+D)^{-1}\right)=\operatorname{det}(C \Omega+D)^{c / 2} W_{g}(\Omega),
$$

under the action of $\left(\begin{array}{ll}A & B \\ C & D\end{array}\right) \in \operatorname{Sp}(2 g, \mathbb{Z})$.

Property (B.2) implies, in particular, that $W$ only depends on the multiplicative periods $\mathrm{e}^{2 \pi \mathrm{i} \Omega_{i j}}$ and one can compose $W$ with (A.22) and (A.23) to define a function $\hat{W}$ on the Schottky space. The Schottky uniformization describes each Riemann surface as a quotient of the Riemann sphere $\hat{\mathbb{C}}$ by a discrete group, so that the coordinates on $\hat{\mathbb{C}}$ canonically define a system of local complex coordinates on the Riemann surface. It is tempting to conjecture that $\hat{W}$ is exactly the partition function $\hat{Z}_{g}$ with respect to such coordinates. This is not true, and the more general relation holds

$$
\hat{Z}_{g}=\frac{\hat{W}_{g}}{F_{g}^{c / 2}}
$$

for a certain holomorphic function $F_{g}$ on $\mathfrak{S}_{g}$. It is clear that the function $F_{g}$ does not depend on the theory in question, so that it may be computed for some particular conformal field theory. For example, by considering the conformal field theories associated to even unimodular lattices, it is natural to conjecture that $F_{g}$ is the function defined in [41], given by

$$
F_{g}=\prod_{m=1}^{\infty} \prod_{\gamma \neq 1}\left(1-p_{\gamma}^{m}\right)
$$

where $p_{\gamma}$ is the multiplier of $\gamma$ and the product runs over all the primitive classes in $\Gamma$, i.e., the $\operatorname{PSL}(2, \mathbb{C})$-conjugacy classes such that $\gamma$ is not conjugated to any power $\tilde{\gamma}^{n}, n>1$. This infinite product converges on a certain open subset of $\mathfrak{S}_{g}$, and $F_{g}$ can be analytically continued to the whole $\mathfrak{S}_{g}$ as a holomorphic function. Similarly to the case of multiplicative periods, the function $F_{2}$ can be written as a series in $p_{1}, p_{2}$. For our purposes, we need the power $F_{2}^{-12}$, given by

$$
F_{2}^{-12}=\sum_{m, n=0}^{\infty} \sum_{r=-m-n}^{m+n} b(m, n,|r|) p_{1}^{m} p_{2}^{n} x^{r}
$$

where the coefficients $b(m, n, r)=b(n, m, r)$ for $m, n \leq 7$ are listed in tables 5 and 6 . 


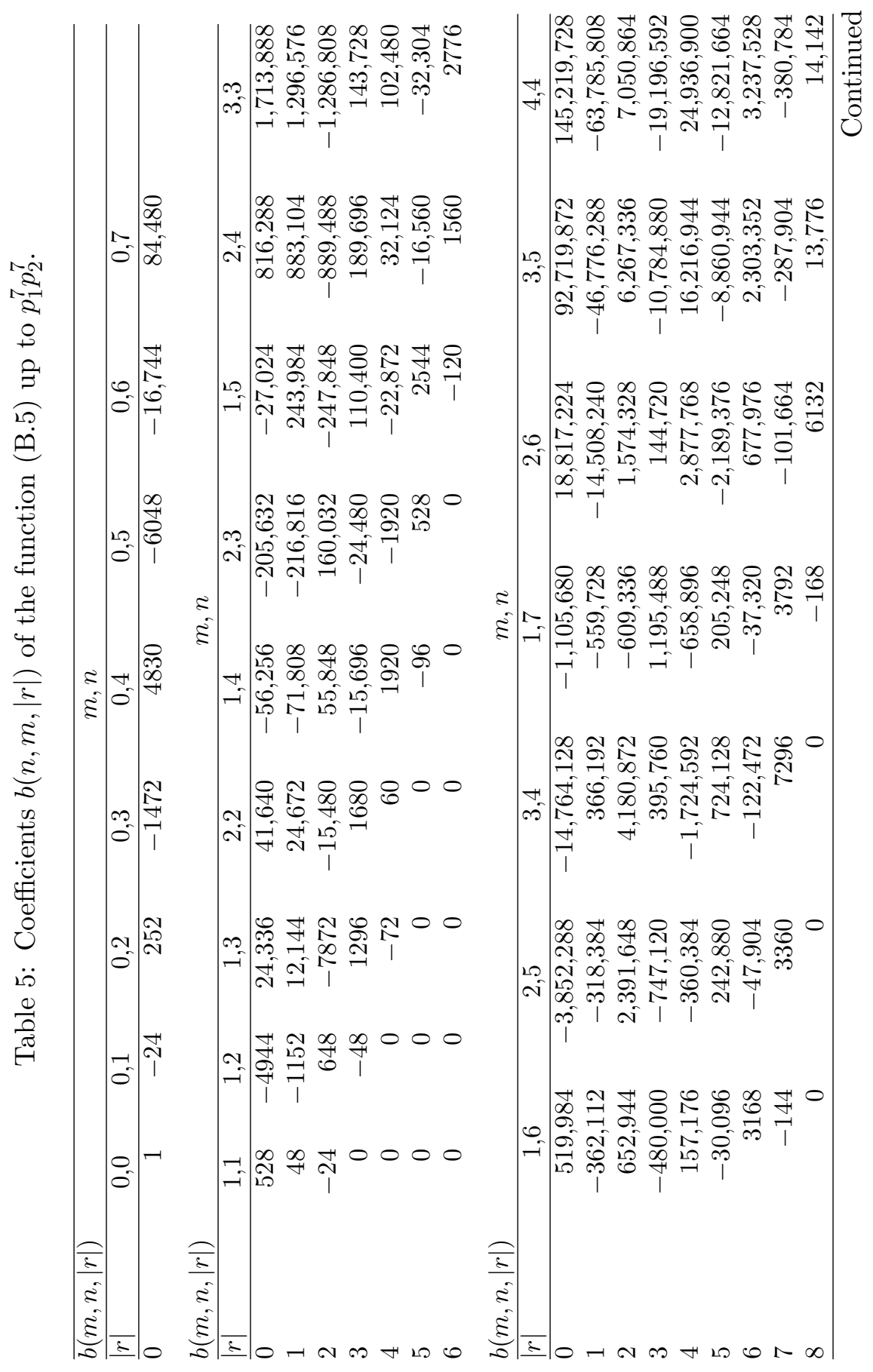


346 Matthias R. Gaberdiel, Christoph A. Keller and Roberto Volpato

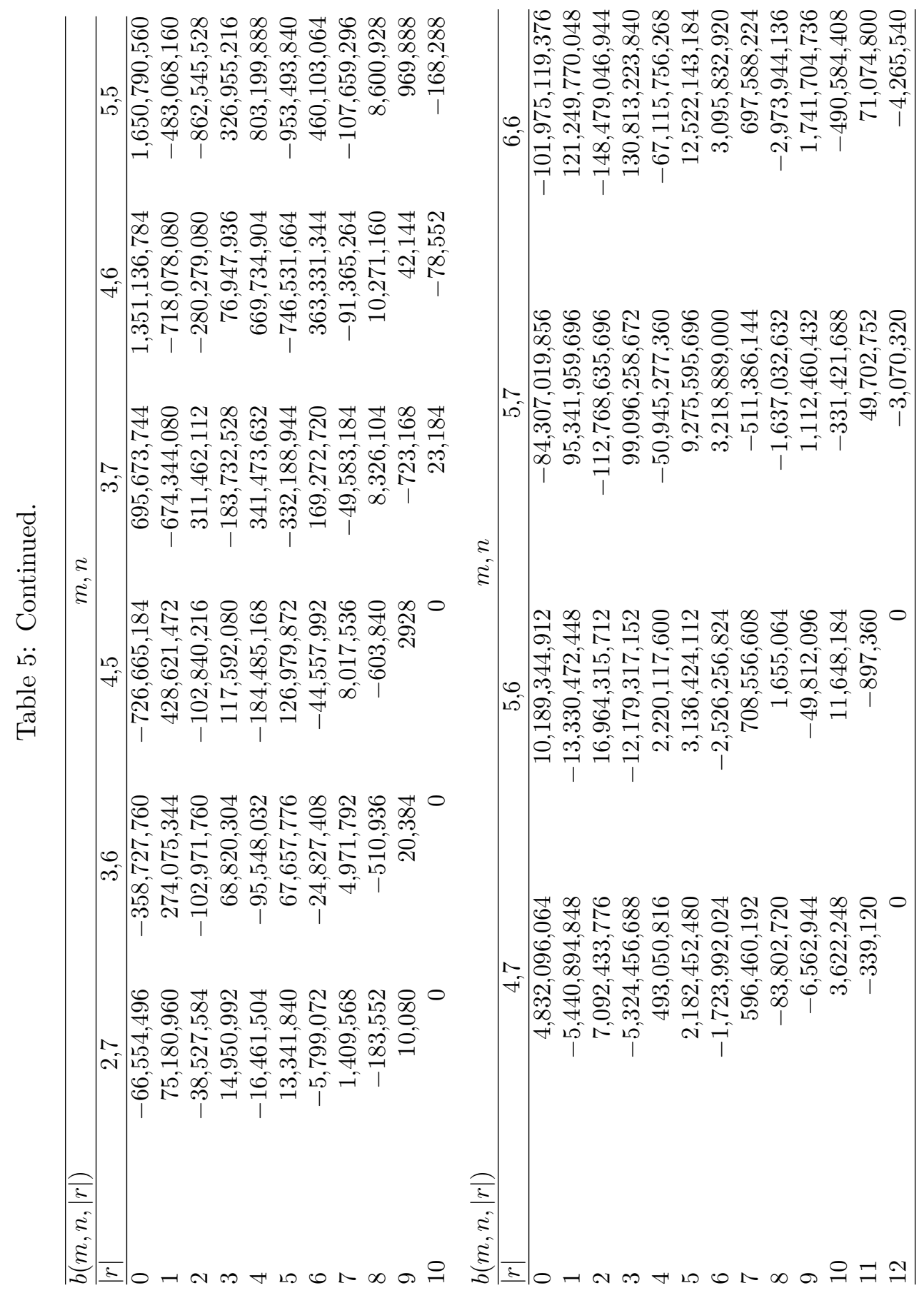


Table 6: Coefficients $b(n, m,|r|)$ of the function (B.5) up to $p_{1}^{7} p_{2}^{7}$.

\begin{tabular}{lrr}
\hline$b(m, n,|r|)$ & \multicolumn{2}{c}{$m, n$} \\
\hline$|r|$ & \multicolumn{2}{c}{$6,7,7$} \\
\hline 0 & $172,479,560,352$ & $3,128,606,566,176$ \\
1 & $-310,507,917,168$ & $-2,134,241,509,440$ \\
2 & $580,363,077,120$ & $-86,143,852,200$ \\
3 & $-687,760,594,368$ & $1,889,752,759,536$ \\
4 & $507,099,802,584$ & $-2,172,090,239,616$ \\
5 & $-235,110,351,024$ & $1,435,905,509,328$ \\
6 & $90,844,794,528$ & $-861,256,923,216$ \\
7 & $-63,838,287,456$ & $741,491,756,592$ \\
8 & $53,462,494,704$ & $-656,667,020,112$ \\
9 & $-30,023,682,864$ & $420,804,461,904$ \\
10 & $10,521,776,736$ & $-181,869,137,376$ \\
11 & $-2,257,032,864$ & $52,175,288,688$ \\
12 & $273,215,976$ & $-9,563,257,536$ \\
13 & $-14,342,640$ & $1,014,591,120$ \\
14 & & $-47,275,560$ \\
\hline
\end{tabular}

\section{Appendix C. Partition function coefficients ad 2g-point functions on the sphere}

A holomorphic function on the Schottky space $\mathfrak{S}_{g}$ can be expanded in a power series in the multipliers $p_{1}, \ldots, p_{g}$

$$
\hat{Z}_{g}=\sum_{h_{1}, \ldots, h_{g}} p_{1}^{h_{1}} \cdots p_{g}^{h_{g}} C_{h_{1}, \ldots, h_{g}}\left(a_{3}, \ldots, a_{g}, r_{2}, \ldots, r_{g}\right)
$$

where we set $a_{1}=0, r_{1}=\infty, a_{2}=1$. If $\hat{Z}_{g}$ is the genus $g$ partition function of a consistent conformal field theory, the functions $C_{h_{1}, \ldots, h_{g}}$ have a natural interpretation as $2 g$-point functions on the sphere. In this section, we will give a heuristic justification of this relationship, following Segal's approach to conformal field theories [40].

In Segal's approach we can define amplitudes for any Riemann surface with parametrized boundary circles $C_{i}$. The degrees of freedom of each boundary circle are labelled by the vector space $\mathcal{H}$ of states; thus any such amplitude also depends on the states $\phi_{i} \in \mathcal{H}$ that are associated to the boundary circles $C_{i}$. (From a string theory perspective, the state $\phi_{i}$ describes the external string state that is inserted at $C_{i}$.) Let us denote these 
348 Matthias R. Gaberdiel, Christoph A. Keller and Roberto Volpato

amplitudes by

$$
\left\langle\prod_{i} \phi_{i}\left[C_{i}\right]\right\rangle_{\mathcal{D}}
$$

where $\mathcal{D}$ denotes a Riemann surface with boundary $\partial \mathcal{D}=\cup_{i} C_{i}$.

In the following we shall usually think of $\mathcal{D}$ as a subset of the complex plane. We shall furthermore characterize the parametrized boundary circle $C_{i}$ by the Möbius transformation $\gamma_{i}$ that maps the standard circle

$$
C:=\{z \in \mathbb{C}:|z|=1\}
$$

to $C_{i}$, i.e., $C_{i}=\gamma_{i}(C)$. Then we can identify the amplitude (C.2) with

$$
\left\langle\prod_{i} \phi_{i}\left[C_{i}\right]\right\rangle_{\mathcal{D}}=\left\langle\prod_{i} V\left(U\left(\gamma_{i}\right) \phi_{i}, \gamma_{i}(0)\right)\right\rangle
$$

where the amplitude on the right-hand side is the standard amplitude in conformal field theory, and

$$
U(\gamma)=\gamma^{\prime}(0)^{L_{0}} \mathrm{e}^{L_{1} \frac{\gamma^{\prime \prime}(0)}{2 \gamma^{\prime}(0)}}
$$

The advantage of Segal's approach is that it allows one to glue Riemann surfaces together along boundary circles. Suppose $\mathcal{D}^{(1)}$ and $\mathcal{D}^{(2)}$ are two Riemann surfaces with boundary circles $C_{i}^{(1)}$ and $C_{j}^{(2)}$, respectively. By the usual plumbing fixture construction we can then define a Riemann surface $\mathcal{D}$ by identifying a parametrized boundary circle of $\mathcal{D}^{(1)}$, say $C_{1}^{(1)}=\gamma_{(1)} C$, with a parametrized boundary circle of $\mathcal{D}^{(2)}$, say $C_{1}^{(2)}=\gamma_{(2)} C$. This procedure is well-defined provided that

$$
\gamma_{(1)}^{-1} \gamma_{(2)}: C \rightarrow \bar{C}
$$

reverses the orientation of the standard circle. Here $\bar{C}$ denotes the standard circle with the opposite orientation, so that $\bar{C}=\hat{\gamma}(C)$ with

$$
\hat{\gamma}(z)=\frac{1}{z} \text {. }
$$

If this is the case, then we can identify the circles $C_{1}^{(1)}$ and $\bar{C}_{1}^{(2)}=\gamma_{(2)} \hat{\gamma} C$ via

$$
\gamma_{(1)} \hat{\gamma} \gamma_{(2)}^{-1}: \bar{C}_{1}^{(2)} \rightarrow C_{1}^{(1)}
$$


and the maps $\gamma_{(1)} \hat{\gamma}$ and $\gamma_{(2)}$ define local analytic coordinates on a neighbourhood of $C_{1}^{(1)} \equiv \bar{C}_{1}^{(2)}$ in $\mathcal{D}$. The amplitude on the $\mathcal{D}$ is then simply

$$
\begin{aligned}
& \left\langle\prod_{i \geq 2} \phi_{i}\left[C_{i}^{(1)}\right] \prod_{j \geq 2} \phi_{j}\left[C_{j}^{(2)}\right]\right\rangle_{\mathcal{D}} \\
& \quad=\sum_{\psi_{1}, \psi_{2}} G_{\psi_{1}, \psi_{2}}^{-1}\left\langle\psi_{1}\left[C_{1}^{(1)}\right] \prod_{i \geq 2} \phi_{i}\left[C_{i}^{(1)}\right]\right\rangle_{\mathcal{D}_{1}}\left\langle\psi_{2}\left[C_{1}^{(2)}\right] \prod_{j \geq 2} \phi_{j}\left[C_{j}^{(2)}\right]\right\rangle_{\mathcal{D}_{2}}{ }^{\prime}
\end{aligned}
$$

where

$$
G_{\phi \psi}=\lim _{z \rightarrow \infty}\left\langle V\left(z^{2 L_{0}} \mathrm{e}^{-z L_{1}} \phi, z\right) V(\psi, 0)\right\rangle
$$

is the metric on the space of states (with $G_{\phi \psi}^{-1}$ the inverse metric).

To illustrate this general construction, consider the annulus

$$
\mathcal{A}:=\{z \in \mathbb{C}:|q|<|z|<1\}
$$

for some $q \in \mathbb{C}, 0<|q|<1$. The boundary components are $\bar{C}=\hat{\gamma}(C)$ and $\gamma_{q}(C)$, where $\gamma_{q}(z)=q z$. The amplitudes on $\mathcal{A}$ are defined as

$$
\left\langle\phi[\hat{\gamma} C] \psi\left[\gamma_{q} C\right]\right\rangle_{\mathcal{A}}=\lim _{z \rightarrow \infty}\left\langle V\left(z^{2 L_{0}} \mathrm{e}^{-z L_{1}} \phi, z\right) V\left(q^{L_{0}} \psi, 0\right)\right\rangle .
$$

Using the prescription (C.9) to glue the boundary circles of the annulus to form the torus with modular parameter $q$ then leads to

$$
\begin{aligned}
& \sum_{\phi, \psi} G_{\phi, \psi}^{-1}\left\langle\phi\left[\gamma_{q} C\right] \phi[\hat{\gamma} C]\right\rangle_{\mathcal{A}} \\
& \quad=\sum_{\phi, \psi} G_{\phi, \psi}^{-1} \lim _{z \rightarrow \infty}\left\langle V\left(z^{2 L_{0}} \mathrm{e}^{-z L_{1}} \phi, z\right) V\left(q^{L_{0}} \psi, 0\right)\right\rangle=\operatorname{Tr}_{\mathcal{H}}\left(q^{L_{0}}\right),
\end{aligned}
$$

which is indeed the expected result.

Let us now consider the case of a Riemann surface of genus $g$, uniformized by a Schottky group $\Gamma$ with generators $\gamma_{1}, \ldots, \gamma_{g}$, and let $p_{i}, a_{i}$ and $r_{i}$ be the multiplier, and the attractive and repelling fixed points of $\gamma_{i}$, respectively. Let us define the Möbius transformations

$$
\gamma_{a_{i}, r_{i}}(z)=\frac{r_{i} z+a_{i}}{z+1}
$$

satisfying $\gamma_{a_{i}, r_{i}}(0)=a_{i}$ and $\gamma_{a_{i}, r_{i}}(\infty)=r_{i}$, so that the generators of $\Gamma$ can be written as

$$
\gamma_{i}=\gamma_{a_{i}, r_{i}} \gamma_{p_{i}} \gamma_{a_{i}, r_{i}}^{-1}, \quad i=1, \ldots, g
$$


350 Matthias R. Gaberdiel, Christoph A. Keller and Roberto Volpato

where $\gamma_{p_{i}}$ is defined as before by $\gamma_{p}(z)=p z$. A fundamental domain for $\Gamma$ is given by

$$
\mathcal{D}:=\hat{\mathbb{C}} \backslash \bigcup_{i=1}^{g}\left(D_{i} \cup D_{-i}\right),
$$

where

$$
\begin{aligned}
D_{i} & =\left\{z \in \mathbb{C}: \frac{\left|z-a_{i}\right|}{\left|z-r_{i}\right|}<\left|R_{i}\right|\right\}=\gamma_{a_{i} r_{i}} \gamma_{R_{i}}(D), \\
D_{-i} & =\left\{z \in \mathbb{C}: \frac{\left|z-r_{i}\right|}{\left|z-a_{i}\right|}<\left|R_{-i}\right|\right\}=\gamma_{a_{i} r_{i}} \hat{\gamma} \gamma_{R_{-i}}(D),
\end{aligned}
$$

and $D$ is the unit disc,

$$
D=\{z \in \mathbb{C}:|z|<1\}
$$

Here $R_{i}, R_{-i} \in \mathbb{C}$ are chosen such that

$$
R_{-i} R_{i}=p_{i}
$$

and all disks $D_{i}, D_{-i}, i=1, \ldots, g$ are disjoint. (Such $R_{i}, R_{-i}$ exist only if Equation (A.21) is satisfied). The boundary $\partial \mathcal{D}=\bigcup_{i} C_{i} \cup \bar{C}_{-i}$ has components

$$
C_{i}=\gamma_{a_{i}, r_{i}} \gamma_{R_{i}} C, \quad \bar{C}_{-i}=\gamma_{a_{i}, r_{i}} \hat{\gamma} \gamma_{R_{-i}} C
$$

We note that

$$
C_{-i} \equiv \overline{\bar{C}}_{-i}=\gamma_{a_{i}, r_{i}} \hat{\gamma} \gamma_{R_{-i}} \hat{\gamma} C=\gamma_{a_{i}, r_{i}} \gamma_{R_{-i}}^{-1} C
$$

since $\hat{\gamma} \gamma_{R_{-i}} \hat{\gamma}=\gamma_{1 / R_{-i}}=\gamma_{R_{-i}}^{-1}$. The Riemann surface can be obtained by gluing each $C_{i} \equiv C_{-i}$ according to the general procedure described above. In this case, because of (C.15) and (C.20), the identification map (C.8) is simply $\gamma_{i}: C_{-i} \rightarrow C_{i}$, in agreement with (A.16). Using the gluing prescription (C.9), the partition function $\hat{Z}_{g}$ is then

$$
\begin{aligned}
\hat{Z}_{g} & =\sum_{\phi_{i} \psi_{i} \in \mathcal{H}} \prod_{i} G_{\phi_{i} \psi_{i}}^{-1}\left\langle\prod_{i} \phi_{i}\left[C_{i}\right] \psi_{i}\left[\bar{C}_{-i}\right]\right\rangle_{\mathcal{D}} \\
& =\sum_{\phi_{i} \psi_{i} \in \mathcal{H}} \prod_{i} G_{\phi_{i} \psi_{i}}^{-1}\left\langle\prod_{i} V\left(U\left(\gamma_{a_{i}, r_{i}}\right) R_{i}^{L_{0}} \phi_{i}, a_{i}\right) V\left(U\left(\gamma_{a_{i}, r_{i}} \hat{\gamma}\right) R_{-i}^{L_{0}} \psi_{i}, r_{i}\right)\right\rangle .
\end{aligned}
$$


If we choose $\phi_{i}, \psi_{i}$ to be eigenvectors of $L_{0}$ and use (C.20), we finally obtain (C.1), with

$$
C_{h_{1}, \ldots, h_{g}}=\sum_{\phi_{i}, \psi_{i} \in \mathcal{H}_{h_{i}}} \prod_{i=1}^{g} G_{\phi_{i} \psi_{i}}^{-1}\left\langle\prod_{i=1}^{g} V^{\mathrm{in}}\left(\phi_{i}, a_{i}\right) V^{\mathrm{out}}\left(\psi_{i}, r_{i}\right)\right\rangle
$$

where

$$
\begin{aligned}
V^{\text {in }}\left(\phi, a_{i}\right) & =V\left(U\left(\gamma_{a_{i}, r_{i}}\right) \phi, a_{i}\right)=V\left(\left(r_{i}-a_{i}\right)^{L_{0}} \mathrm{e}^{-L_{1}} \phi, a_{i}\right), \\
V^{\text {out }}\left(\psi, r_{i}\right) & =V\left(U\left(\gamma_{a_{i}, r_{i}} \hat{\gamma}\right) \psi, r_{i}\right)=V\left(\left(r_{i}-a_{i}\right)^{L_{0}} \mathrm{e}^{L_{1}} \psi, r_{i}\right) .
\end{aligned}
$$

Note that $\hat{Z}_{g}$ is independent of the specific choice of $R_{i}, R_{-i}$ satisfying (C.20). Equivalently, $C_{h_{1}, \ldots, h_{g}}$ is not affected by any replacement $\gamma_{a_{i}, r_{i}} \rightarrow$ $\gamma_{a_{i}, r_{i}} \gamma_{t}$ with $t \in \mathbb{C}^{*}$, in the definition of $V^{\text {in }}$ and $V^{\text {out }}$. When all states in $\mathcal{H}_{h_{1}}, \ldots, \mathcal{H}_{h_{g}}$ are quasiprimaries, (C.24) simplifies to

(C.27) $\mathcal{C}_{h_{1}, \ldots, h_{g}}=\prod_{i=1}^{g}\left(r_{i}-a_{i}\right)^{2 h_{i}} \sum_{\phi_{i}, \psi_{i} \in \mathcal{H}_{h_{i}}} \prod_{i=1}^{g} G_{\phi_{i} \psi_{i}}^{-1}\left\langle\prod_{i=1}^{g} V\left(\phi_{i}, r_{i}\right) V\left(\psi_{i}, a_{i}\right)\right\rangle$.

At genus $g=2$, Equation (C.24) can be written as

$$
\begin{aligned}
C_{h_{1}, h_{2}}(x)= & \sum_{\phi_{i}, \psi_{i} \in \mathcal{H}_{h_{i}}} G_{\phi_{1} \psi_{1}}^{-1} G_{\phi_{2} \psi_{2}}^{-1} \\
& \left\langle V^{\text {out }}\left(\psi_{1}, \infty\right) V^{\text {out }}\left(\psi_{2}, x\right) V^{\text {in }}\left(\phi_{2}, 1\right) V^{\text {in }}\left(\phi_{1}, 0\right)\right\rangle,
\end{aligned}
$$

where

(C.29) $V^{\text {out }}\left(\psi_{1}, \infty\right)=V\left(U(\hat{\gamma}) \psi_{1}, \hat{\gamma}(0)\right), \quad V^{\text {in }}\left(\phi_{1}, 0\right)=V\left(\phi_{1}, 0\right)$,

(C.30) $V^{\text {out }}\left(\psi_{2}, x\right)=V\left(U\left(\gamma_{1, x} \hat{\gamma}\right) \psi_{2}, x\right), \quad V^{\text {in }}\left(\phi_{2}, 1\right)=V\left(U\left(\gamma_{1, x}\right) \phi_{2}, 1\right)$.

As mentioned before, we can equivalently replace $\gamma_{1, x}$ by any $\gamma_{1, x} \gamma_{t}$ for $t \in \mathbb{C}^{*}$. In particular, if we replace $\gamma_{1, x}$ by the involution

$$
\tilde{\gamma}_{1, x}(z)=\frac{z-1}{z / x-1}=\gamma_{1, x} \gamma_{-1 / x}
$$


352 Matthias R. Gaberdiel, Christoph A. Keller and Roberto Volpato

the symmetries of $C_{h_{1}, h_{2}}(x)$ are immediate. Indeed, using the invariance of the four-point amplitude under the Möbius transformation $\tilde{\gamma}_{1, x}$, we have

$$
\begin{aligned}
C_{h_{1}, h_{2}}(x)= & \sum_{\phi_{i}, \psi_{i} \in \mathcal{H}_{h_{i}}} G_{\phi_{1} \psi_{1}}^{-1} G_{\phi_{2} \psi_{2}}^{-1} \\
& \left\langle V\left(U\left(\tilde{\gamma}_{1, x} \hat{\gamma}\right) \psi_{1}, x\right) V\left(U(\hat{\gamma}) \psi_{2}, \infty\right) V\left(\phi_{2}, 0\right) V\left(U\left(\tilde{\gamma}_{1, x}\right) \phi_{1}, 1\right)\right\rangle \\
= & \sum_{\phi_{i}, \psi_{i} \in \mathcal{H}_{h_{i}}} G_{\phi_{1} \psi_{1}}^{-1} G_{\phi_{2} \psi_{2}}^{-1} \\
& \left\langle V^{\text {out }}\left(\psi_{1}, x\right) V^{\text {out }}\left(\psi_{2}, \infty\right) V^{\text {in }}\left(\phi_{2}, 0\right) V^{\text {in }}\left(\phi_{1}, 1\right)\right\rangle \\
= & C_{h_{2}, h_{1}}(x) .
\end{aligned}
$$

Similarly, applying the Möbius transformation $\hat{\gamma}$ and noting that $\hat{\gamma} \tilde{\gamma}_{1, x} \hat{\gamma}=\tilde{\gamma}_{1 / x, 1}$, we obtain

$$
\begin{aligned}
C_{h_{1}, h_{2}}(x)= & \sum_{\phi_{i}, \psi_{i} \in \mathcal{H}_{h_{i}}} G_{\phi_{1} \psi_{1}}^{-1} G_{\phi_{2} \psi_{2}}^{-1} \\
& \left\langle V\left(\psi_{1}, 0\right) V\left(U\left(\tilde{\gamma}_{1 / x, 1}\right) \psi_{2}, 1 / x\right) V\left(U\left(\tilde{\gamma}_{1 / x, 1} \hat{\gamma}\right) \phi_{2}, 1\right) V\left(U(\hat{\gamma}) \phi_{1}, \infty\right)\right\rangle \\
= & \sum_{\phi_{i}, \psi_{i} \in \mathcal{H}_{h_{i}}} G_{\phi_{1} \psi_{1}}^{-1} G_{\phi_{2} \psi_{2}}^{-1} \\
& \left\langle V^{\text {in }}\left(\psi_{1}, 0\right) V^{\text {in }}\left(\psi_{2}, 1 / x\right) V^{\text {out }}\left(\phi_{2}, 1\right) V^{\text {out }}\left(\phi_{1}, \infty\right)\right\rangle \\
= & C_{h_{1}, h_{2}}(1 / x) .
\end{aligned}
$$

The same argument also applies to the quasiprimary functions, defined in $(3.2)$.

\section{Appendix D. Technicalities}

\section{D.1. The relation between $\mathcal{D}$ and $\mathcal{C}$}

In this appendix we will prove, using the associativity of the OPE, that one can always express $\mathcal{C}_{h_{1}, h_{2} ; l}^{(*)}$ in terms of $\mathcal{D}_{h_{1}^{\prime}, h_{2}^{\prime} ; l^{\prime}}$ with $h_{1}^{\prime} \leq h_{1}, h_{2}^{\prime} \leq h_{2}$ and $l^{\prime} \leq l$ and vice versa. In fact, we will prove that the linear spans of these coefficients are related as

$$
\left\langle\mathcal{C}_{h_{1}, h_{2}^{\prime} ; l}^{(*)}\right\rangle_{\substack{l=0, \ldots, L \\ h_{2}^{\prime} \leq h_{2}}}=\left\langle\mathcal{D}_{h_{1}, h_{2}^{\prime} ; l}\right\rangle_{\substack{l=0, \ldots, L \\ h_{2}^{\prime} \leq h_{2}}}
$$


To do this it is useful to introduce yet another set of invariants $\mathcal{C}_{h_{1}, h_{2} ; l}^{(1)}$ as

$$
\mathcal{C}_{h_{1}, h_{2}}(x)=\sum_{l=0}^{\infty}(x-1)^{l} \mathcal{C}_{h_{1}, h_{2} ; l}^{(1)}
$$

Using the same arguments as in Section 3.1.1 it follows that these invariants can be interpreted as

$$
\mathcal{C}_{h_{1}, h_{2} ; l}^{(1)}:=\sum_{\phi_{2}, \psi_{2} \in \mathcal{H}_{h_{2}}^{q p}} G_{\phi_{2} \psi_{2}}^{-1} \operatorname{Tr}_{\mathcal{H}_{h_{1}}}\left(V_{0}\left(V_{h_{2}-l}\left(\phi_{2}\right) \psi_{2}\right)\right) .
$$

In order to apply these results also to the case of Section 4, we will not assume that $\mathcal{H}$ is a consistent self-dual conformal field theory, but just the representation of a chiral algebra $\mathcal{A}$, so that (4.1) holds for some $B>0$ (that is, all the fields up to weight $B$ are in the vacuum representation $\mathcal{H}^{(0)}$ of a consistent chiral algebra). Notice that (D.3) still makes sense for $h_{2}<B$, because $V_{h_{2}-l}\left(\phi_{2}\right) \psi_{2} \in \mathcal{H}^{(0)}$, so we will restrict ourselves to this case.

Using a similar argument as in (3.7) one shows that

$$
\left\langle\mathcal{C}_{h_{1}, h_{2} ; l}^{(*)}\right\rangle_{l=0, \ldots, L}=\left\langle\mathcal{C}_{h_{1}, h_{2} ; 2 l}^{(1)}\right\rangle_{l=0, \ldots, L}
$$

Note the appearance of $2 l$ for the $\mathcal{C}_{h_{1}, h_{2} ; l}^{(1)}$, which comes from the fact that the leading power in the term $\mathcal{C}_{h_{1}, h_{2} ; l}^{(*)}$ is $(x-1)^{2 l}$. We now want to show that the invariants $\mathcal{C}_{h_{1}, h_{2} ; 2 l}^{(1)}$ can be written in terms of the invariants $\mathcal{D}_{h_{1}, h_{2} ; l}$. Let $\phi_{2}, \psi_{2} \in \mathcal{H}_{h_{2}}^{(0)}$ be quasiprimary states and consider

$$
V_{0}\left(V_{h_{2}-2 l}\left(\phi_{2}\right) \psi_{2}\right)=\sum_{\phi, \psi \in \mathcal{H}_{2 l} \cap \mathcal{H}^{(0)}} G_{\phi \psi}^{-1} V_{0}(\phi)\left\langle\psi \mid V_{h_{2}-2 l}\left(\phi_{2}\right) \psi_{2}\right\rangle,
$$

where $\langle\phi \mid \psi\rangle \equiv G_{\phi \psi}$. The sum on the right-hand side can be taken over states of the form $\psi=L_{-1}^{n} \psi^{\prime}$ and $\phi=L_{-1}^{m} \phi^{\prime}$, with $m, n \geq 0$ and $\phi^{\prime}$ and $\psi^{\prime}$ quasiprimary states of weight $2 l-n$ and $2 l-m$. Note that

$$
\begin{aligned}
G_{\phi \psi} & =\left\langle\phi^{\prime} \mid L_{1}^{m} L_{-1}^{n} \psi^{\prime}\right\rangle=\delta_{m n} n !(4 l-2 n) \cdots(4 l-n-1) G_{\phi^{\prime} \psi^{\prime}}, \\
V_{0}(\phi) & =V_{0}\left(L_{-1}^{m} \phi^{\prime}\right)=(-1)^{m}(2 l-1)(2 l-2) \cdots(2 l-m) V_{0}\left(\phi^{\prime}\right),
\end{aligned}
$$

and

$$
\left\langle\psi \mid V_{h_{2}-2 l}\left(\phi_{2}\right) \psi_{2}\right\rangle=\left\langle\psi^{\prime} \mid L_{1}^{n} V_{h_{2}-2 l}\left(\phi_{2}\right) \psi_{2}\right\rangle
$$

$$
=(2 l-1)(2 l-2) \cdots(2 l-n)\left\langle\psi^{\prime} \mid V_{h_{2}-(2 l-n)}\left(\phi_{2}\right) \psi_{2}\right\rangle .
$$


354 Matthias R. Gaberdiel, Christoph A. Keller and Roberto Volpato

It thus follows that $V_{0}\left(V_{h_{2}-2 l}\left(\phi_{2}\right) \psi_{2}\right)$ can be expressed as a linear combination of

$$
\begin{aligned}
& \sum_{\phi, \psi \in \mathcal{H}_{l^{\prime}}^{q p} \cap \mathcal{H}^{(0)}} G_{\phi \psi}^{-1} V_{0}(\phi)\left\langle\psi \mid V_{h_{2}-l^{\prime}}\left(\phi_{2}\right) \psi_{2}\right\rangle \\
= & \sum_{\phi, \psi \in \mathcal{H}_{l^{\prime}}^{q p} \cap \mathcal{H}^{(0)}} G_{\phi \psi}^{-1} V_{0}(\phi) \lim _{z \rightarrow \infty} z^{2 l^{\prime}}\left\langle\psi(z) \phi_{2}(1) \psi_{2}(0)\right\rangle
\end{aligned}
$$

with $l^{\prime}=0, \ldots, 2 l$. Applying a fractional linear transformation to the last correlator, we can exchange 1 and $\infty$ while keeping 0 fixed. Because all the states in this correlator are quasiprimary, we have simply

$$
\begin{aligned}
& \lim _{z \rightarrow \infty} z^{2 l^{\prime}}\left\langle\psi(z) \phi_{2}(1) \psi_{2}(0)\right\rangle \\
& \quad=(-1)^{l^{\prime}} \lim _{\zeta \rightarrow \infty} \zeta^{2 h_{2}}\left\langle\phi_{2}(\zeta) \psi(1) \psi_{2}(0)\right\rangle=(-1)^{l^{\prime}}\left\langle\phi_{2} \mid V_{0}(\psi) \psi_{2}\right\rangle .
\end{aligned}
$$

Thus $\mathcal{C}_{h_{1}, h_{2} ; 2 l}^{(1)}, h_{2}<B$ is a linear combination of

$$
\begin{aligned}
\sum_{\substack{\phi_{2}, \psi_{2} \in \mathcal{H}_{h_{2}}^{q p} \\
\phi, \psi \in \mathcal{H}_{2 l^{\prime}}^{q p} \cap \mathcal{H}^{(0)}}} G_{\phi \psi}^{-1} G_{\phi_{2} \psi_{2}}^{-1} \operatorname{Tr}_{\mathcal{H}_{h_{1}}} V_{0}(\phi)\left\langle\phi_{2} \mid V_{0}(\psi) \psi_{2}\right\rangle \\
=\sum_{\phi, \psi \in \mathcal{H}_{2 l^{\prime}}^{q p} \cap \mathcal{H}^{(0)}} G_{\phi \psi}^{-1} \operatorname{Tr}_{\mathcal{H}_{h_{1}}} V_{0}(\phi) \operatorname{Tr}_{\mathcal{H}_{h_{2}}^{q p}}\left(V_{0}(\psi)\right)
\end{aligned}
$$

for $l^{\prime}=0, \ldots, l$, and therefore, by (D.7), it is also a linear combination of

$$
\sum_{\phi, \psi \in \mathcal{H}_{2 l^{\prime}} \cap \mathcal{H}^{(0)}} G_{\phi \psi}^{-1} \operatorname{Tr}_{\mathcal{H}_{h_{1}}} V_{0}(\phi) \operatorname{Tr}_{\mathcal{H}_{h_{2}}^{q p}}\left(V_{0}(\psi)\right), \quad l^{\prime}=0, \ldots, 2 l .
$$

Next we observe that for any $\psi \in \mathcal{H}^{(0)}$, we have

$$
\operatorname{Tr}_{\mathcal{H}_{h_{2}}}\left(V_{0}(\psi)\right)=\sum_{n=0}^{h_{2}} \operatorname{Tr}_{L_{-1}^{n} \mathcal{H}_{h_{2}-n}^{q p}}\left(V_{0}(\psi)\right)=\sum_{n=0}^{h_{2}} c\left(h_{2}, n\right) \operatorname{Tr}_{\mathcal{H}_{h_{2}-n}^{q p}}\left(V_{0}(\psi)\right)
$$

for some coefficients $c\left(h_{2}, n\right)$. These identities can be inverted to obtain $\operatorname{Tr}_{\mathcal{H}_{h_{2}}^{q p}}\left(V_{0}(\psi)\right)$ as a linear combination of $\operatorname{Tr}_{\mathcal{H}_{h_{2}^{\prime}}}\left(V_{0}(\psi)\right)$ with $h_{2}^{\prime} \leq h_{2}$. 
Furthermore, by (3.11), for all $N \geq 0$ we have

$$
\bigoplus_{n=0}^{N} \mathcal{H}_{n}=\bigoplus_{n=0}^{N} \mathcal{H}_{[n]}
$$

Thus, by (D.11), $\mathcal{C}_{h_{1}, h_{2} ; 2 l}^{(1)}$ is a linear combination of $\mathcal{D}_{h_{1}, h_{2}^{\prime} ; l^{\prime}}$ with $l^{\prime} \leq l$ and $h_{2}^{\prime} \leq h_{2}$. More precisely, for any $h_{2} \leq h_{1}, h_{2} \leq B$ and $L \geq 0$, we have

$$
\left\langle\mathcal{C}_{h_{1}, h_{2}^{\prime} ; 2 l}^{(1)}\right\rangle_{\substack{l=0, \ldots, L \\ h_{2}^{\prime} \leq h_{2}}}=\left\langle\mathcal{D}_{h_{1}, h_{2}^{\prime} ; l}\right\rangle_{\substack{l=0, \ldots, L \\ h_{2}^{\prime} \leq h_{2}}}
$$

Together with (D.4) this then immediately implies (D.1).

\section{D.2. Completeness of the relations}

In this appendix we prove Theorem 3.1. For what follows it will be useful to introduce a lexicographical ordering on the space of coefficients. We say that

$$
\left(h_{1}, h_{2} ; l\right) \prec\left(\hat{h}_{1}, \hat{h}_{2} ; \hat{l}\right)
$$

if (1) $h_{2}<\hat{h}_{2}$, or (2) $h_{2}=\hat{h}_{2}$ and $h_{1}<\hat{h}_{1}$, or (3) $h_{2}=\hat{h}_{2}, h_{1}=\hat{h}_{1}$ and $l<\hat{l}$. We define the relation $\preceq$ in the obvious way.

To prove Theorem 3.1, first note that by (3.8) and (3.14) we can express all invariants $\mathcal{C}_{h_{1}, h_{2} ; l}^{(0)}$ and $\mathcal{D}_{h_{1}, h_{2} ; l}$ as linear combinations of $\mathcal{C}_{h_{1}, h_{2} ; l}^{(*)}$ with $l=0, \ldots, h_{1}+h_{2}$. We then want to prove the following lemma:

Lemma D. 1. Let $\left(h_{1}, h_{2} ; l\right) \notin \mathcal{P}_{k}^{(*)}$. It is then possible to express $\mathcal{C}_{h_{1}, h_{2} ; l}^{(*)}$ in terms of invariants $\mathcal{C}_{h_{1}^{\prime}, h_{2}^{\prime} ; l^{\prime}}^{(*)}$ with $\left(h_{1}^{\prime}, h_{2}^{\prime} ; l^{\prime}\right) \prec\left(h_{1}, h_{2} ; l\right)$.

By repeatedly applying Lemma D.1 it is clear that we can express any $\mathcal{C}^{(*)}$ invariant in terms of the invariants $\mathcal{C}_{h_{1}, h_{2} ; l}^{(*)}$ with $\left(h_{1}, h_{2} ; l\right) \in \mathcal{P}_{k}^{(*)}$. From this Theorem 3.1 follows.

Proof of Lemma 1. For $\left(h_{1}, h_{2} ; l\right) \notin \mathcal{P}_{k}^{(*)}$ it is enough to treat two different cases:

1) $l>h_{2}$ : It follows from (3.8) that we can express $\mathcal{C}_{h_{1}, h_{2} ; l}^{(*)}$ as a linear combination of $\mathcal{C}_{h_{1}, h_{2} ; l^{\prime}}^{(0)}$ with $l^{\prime}=0,1, \ldots, L$ with $L=h_{1}+h_{2}-l<h_{1}$. We can then use (3.15) to rewrite $\mathcal{C}_{h_{1}, h_{2} ; l^{\prime}}^{(0)}=\mathcal{C}_{l^{\prime}, h_{2} ; h_{1}}^{(0)}$. Using (3.8) again, 
these can in turn be expressed in terms of $\mathcal{C}_{l^{\prime}, h_{2} ; l^{\prime \prime}}^{(*)}$, where $\left(l^{\prime}, h_{2} ; l^{\prime \prime}\right) \prec$ $\left(h_{1}, h_{2} ; l\right)$ because $l^{\prime}<h_{1}$.

2) $h_{1}>k+l-w_{l}$ or $h_{2}>h_{1}$ : Using $(3.14)$ we can express $\mathcal{C}_{h_{1}, h_{2} ; l}^{(*)}$ in terms of $\mathcal{D}_{h_{1}^{\prime}, h_{2}^{\prime} ; l^{\prime}}$ with $\left(h_{1}^{\prime}, h_{2}^{\prime} ; l^{\prime}\right) \preceq\left(h_{1}, h_{2} ; l\right)$. Since we can use $(3.14)$ to rewrite the $\mathcal{D}_{h_{1}^{\prime}, h_{2}^{\prime} ; l^{\prime}}$ in terms of $\mathcal{C}_{h_{1}^{\prime \prime}, h_{2}^{\prime \prime} ; l^{\prime \prime}}^{(*)}$ with $\left(h_{1}^{\prime \prime}, h_{2}^{\prime \prime} ; l^{\prime \prime}\right) \preceq\left(h_{1}^{\prime}, h_{2}^{\prime} ; l^{\prime}\right)$, it is clear that we only need to worry about the terms with $\left(h_{1}^{\prime}, h_{2}^{\prime} ; l^{\prime}\right)=$ $\left(h_{1}, h_{2} ; l\right)$. In the case $h_{2}>h_{1}$, we have the obvious relation $\mathcal{D}_{h_{1}, h_{2} ; l}=$ $\mathcal{D}_{h_{2}, h_{1} ; l}$ and $\left(h_{2}, h_{1} ; l\right) \prec\left(h_{1}, h_{2} ; l\right)$. In the case $h_{1}>k+l-w_{l}$, we can use (3.20) to express $\mathcal{D}_{h_{1}, h_{2} ; l}$ as a linear combination of $\mathcal{D}_{h_{1}^{\prime}, h_{2}^{\prime} ; l}$ with $h_{2}^{\prime} \leq h_{1}^{\prime} \leq k+l-w_{l}$ and $h_{2}^{\prime} \leq h_{2}$. From this we see that $h_{1}^{\prime}<h_{1}$, so that after a reconversion to $\mathcal{C}^{(*)}$, using (3.14), the claim of the lemma also follows in this case.

\section{D.3. Free parameters and the slope of effective divisors}

Recall that for $g=2$, the triples $\left(h_{1}, h_{2}, l\right) \in \mathcal{P}_{k}^{(*)}$ satisfy the bound (see $(2.29))$

$$
h_{1}, h_{2} \leq \frac{6}{5} k
$$

In this section we will discuss a similar bound for $\mathcal{P}_{k}(g)$, for general $g$. Our approach is similar to the procedure adopted in [42] in the framework of string theory.

Recall from Appendix B that the genus $g$ partition function of a chiral conformal field theory of central charge $c=24 k$ is a section of the tensor power $\mathcal{L}^{\otimes 12 k}$ of the Hodge bundle on $\mathcal{M}_{g}$, whose fiber at a certain point $\Sigma$ is $\bigwedge^{g} H^{0}\left(\Sigma, K_{\Sigma}\right)$. This line bundle naturally extends to the Deligne-Mumford compactification

$$
\overline{\mathcal{M}}_{g}=\mathcal{M}_{g} \cup \bigcup_{i=0}^{[g / 2]} \Delta_{i}
$$

of the moduli space. Here, a generic element in the boundary component $\Delta_{i}$, $i>0$, is obtained by identifying a point on a curve of genus $i$ with a point on a curve of genus $g-i$; a generic element of $\Delta_{0}$ is obtained by identifying two distinct points on a curve of genus $g-1$.

Let $\operatorname{Pic}\left(\overline{\mathcal{M}}_{g}\right)$ be the group of (isomorphism classes of) holomorphic line bundles on $\overline{\mathcal{M}}_{g}$, equipped with a tensor product and with the inverse given 
by the dual line bundle. The Picard group $\operatorname{Pic}\left(\overline{\mathcal{M}}_{g}\right)$ is isomorphic ${ }^{5}$ to the group of divisor classes on $\overline{\mathcal{M}}_{g}$. Thus we shall from now on adopt an additive notation for this group. Let us define $b^{6}$

$$
\delta_{i}=\left[\Delta_{i}\right], \quad i \neq 1, \quad \delta_{1}=\frac{1}{2}\left[\Delta_{1}\right]
$$

the divisor classes of the boundary components. It can be shown that $\operatorname{Pic}\left(\overline{\mathcal{M}}_{g}\right)$ is generated by

$$
\lambda, \delta_{0}, \ldots, \delta_{\left[\frac{g}{2}\right]},
$$

where $\lambda$ denotes the divisor class of the Hodge bundle $\mathcal{L}$. (For $g>2$ there are no relations, whereas for $g=2$ there is one relation, namely $10 \lambda=\delta_{0}+2 \delta_{1}$.)

It is clear that the elements of the set $\mathcal{P}_{k}(g)$ correspond to a basis of the space of holomorphic sections of $\mathcal{L}^{\otimes 12 k}$. Let $Z$ and $Z^{\prime}$ be two distinct sections of $\mathcal{L}^{\otimes 12 k}$. The divisor $\left(Z-Z^{\prime}\right)$ of their difference can be written as

$$
\left(Z-Z^{\prime}\right)=D+b_{0} \Delta_{0}+\frac{1}{2} b_{1} \Delta_{1}+\sum_{i=2}^{[g / 2]} b_{i} \Delta_{i}
$$

where $D$ is (the closure of) an effective divisor in $\mathcal{M}_{g}$, and $b_{i} \geq 0$. Since $Z-Z^{\prime}$ is again a section of $\mathcal{L}^{\otimes 12 k}$, Equation (D.21) implies

$$
[D]=12 k \lambda-\sum_{i=0}^{[g / 2]} b_{i} \delta_{i}
$$

in terms of divisor classes. Suppose that $Z-Z^{\prime}$ vanishes at order at least $L \geq 0$ in any degeneration limit, that is

$$
L=\min _{i} b_{i}
$$

If we consider any expansion of $Z$ and $Z^{\prime}$ of the form (5.8), this means that

$$
C_{h_{1}, \ldots, h_{3 g-3}}=C_{h_{1}, \ldots, h_{3 g-3}}^{\prime},
$$

\footnotetext{
${ }^{5}$ More precisely, $\operatorname{Pic}\left(\overline{\mathcal{M}}_{g}\right) \otimes \mathbb{Q}$ is isomorphic to the group of rational divisor classes on $\hat{\mathcal{M}}_{g}$ [43].

${ }^{6}$ All the singular curves in $\Delta_{1}$ have a non-trivial automorphism, acting as the involution of the torus with one puncture. For this reason, it is convenient to include a factor $1 / 2$ in the definition of $\delta_{1}$.
} 
358 Matthias R. Gaberdiel, Christoph A. Keller and Roberto Volpato

whenever $h_{i}<L$ for some $i$. Thus, two distinct $Z$ and $Z^{\prime}$ satisfying (D.23) exist if and only if there is at least one element in $\mathcal{P}_{k}(g)$ with $h_{i} \geq L$ for all $i$.

Following [44] we defined the slope $s_{g}$ as

$$
\begin{gathered}
s_{g}=\inf \left\{\frac{a}{\min _{i} b_{i}} \mid a, b_{i}>0, \exists \text { effective divisor } D\right. \text { such that } \\
\left.[D]=a \lambda-\sum_{i=0}^{[g / 2]} b_{i} \delta_{i}\right\} .
\end{gathered}
$$

Then $Z \neq Z^{\prime}$ implies

$$
L \leq \frac{12 k}{s_{g}},
$$

and hence there is no element in $\mathcal{P}_{k}(g)$ with $h_{i}>\frac{12 k}{s_{g}}$ for all $h_{i}$.

For small values of $g$, the value of $s_{g}$ has been determined in [44-46]. A lower bound valid for all $g$ (but in general not sharp) is [47]

$$
s_{g} \geq \frac{60}{g+4} .
$$

Together with (D.26) this then implies that $\mathcal{P}_{k}(g)$ does not contain any elements with

$$
h_{1}, \ldots, h_{3 g-3}>\frac{k}{5}(g+4)
$$

which is the desired inequality.

\section{D.4. Proof of formula (5.18)}

The function $Z_{1}(q)$ is a modular form of weight $12 k$, so that

$$
\frac{Z_{1}(q)}{\Delta^{k}}=\sum_{h=0}^{\infty} n_{h} q^{h-k}
$$

is modular invariant. This implies that it can be written as

$$
\frac{Z_{1}(q)}{\Delta^{k}}=n_{k}+\sum_{t=1}^{k} n_{k-t} T_{t}^{\prime} J(\tau)
$$


where

$$
J(\tau)=j(\tau)-744=\sum_{m=-1}^{\infty} c_{m} q^{m}=q^{-1}+196884 q+\cdots
$$

is the Klein invariant and $T_{t}^{\prime}$ is the Hecke operator

$$
T_{t}^{\prime} F(\tau)=\sum_{d \mid t} \sum_{b=0}^{d-1} F\left(\frac{t \tau+b d}{d^{2}}\right)
$$

If $F$ is modular invariant, then so is $T_{t}^{\prime} F$. Furthermore,

$$
\begin{aligned}
T_{t}^{\prime} J(\tau) & =\sum_{d \mid t} \sum_{m=-1}^{\infty} c_{m} \mathrm{e}^{2 \pi \mathrm{i} m \frac{t \tau}{d^{2}}} \sum_{b=0}^{d-1} \mathrm{e}^{2 \pi \mathrm{i} \frac{m b}{d}} \\
& =\sum_{m=-1}^{\infty} c_{m} \sum_{d \mid(t,|m|)} d \mathrm{e}^{2 \pi \mathrm{i} m \frac{t \tau}{d^{2}}} \\
& =q^{-t}+\sum_{l=1}^{\infty} q^{l} \sum_{d \mid(t, l)} \frac{t}{d} c_{t l / d^{2}}
\end{aligned}
$$

where $(a, b)$ denotes the greatest common divisor. Equation (D.30) follows immediately from these properties of Hecke operators. Using Rademacher's formula, one has the following asymptotic estimate for the coefficients of $J(\tau)$

$$
c_{m} \sim \frac{m^{-3 / 4}}{\sqrt{2}} \mathrm{e}^{4 \pi \sqrt{m}}, \quad m \gg 0 .
$$

Thus, the coefficients $n_{l+k}$ of $Z_{1} / \Delta^{12 k}$ for large $l$ are given by

$$
\begin{aligned}
n_{l+k}= & \sum_{t=1}^{k} n_{k-t} \sum_{d \mid(t, l)} \frac{t}{d} c_{t l / d^{2}} \sim \sum_{t=1}^{k} n_{k-t} \\
& \sum_{d \mid(t, l)} \frac{t}{d} \frac{d^{3 / 2}}{\sqrt{2}(t l)^{3 / 4}} \mathrm{e}^{4 \pi \frac{\sqrt{t l}}{d}} \sim n_{0} \frac{k^{1 / 4}}{\sqrt{2}} l^{-3 / 4} \mathrm{e}^{4 \pi \sqrt{k l}},
\end{aligned}
$$

where in the last step we retained only the leading terms $t=k$ and $d=1$. This then implies Equation (5.18) because $n_{0}=1$ follows from the uniqueness of the vacuum. 
360 Matthias R. Gaberdiel, Christoph A. Keller and Roberto Volpato

\section{References}

[1] G.W. Moore and N. Seiberg, Classical and quantum conformal field theory, Commun. Math. Phys. 123 (1989), 177.

[2] E. Witten, Three-dimensional gravity revisited, arXiv:0706.3359 [hep-th].

[3] A. Maloney and E. Witten, Quantum gravity partition functions in three dimensions, arXiv:0712.0155 [hep-th].

[4] D. Gaiotto and X. Yin, Genus two partition functions of extremal conformal field theories, JHEP 0708 (2007), 029 [arXiv:0707.3437 [hep-th]].

[5] X. Yin, Partition functions of three-dimensional pure gravity, arXiv:0710.2129 [hep-th].

[6] X. Yin, On non-handlebody instantons in 3d gravity, JHEP 0809 (2008), 120 [arXiv:0711.2803 [hep-th]].

[7] D. Friedan and S.H. Shenker, The analytic geometry of two-dimensional conformal field theory, Nucl. Phys. B281 (1987), 509.

[8] M.R. Gaberdiel and R. Volpato, Higher genus partition functions of meromorphic conformal field theories, JHEP 0906 (2009), 048 [arXiv:0903.4107 [hep-th]].

[9] M.R. Gaberdiel, Constraints on extremal self-dual CFTs, JHEP 0711 (2007), 087 [arXiv:0707.4073 [hep-th]].

[10] M.R. Gaberdiel and C.A. Keller, Modular differential equations and null vectors, JHEP 0809 (2008), 079 [arXiv:0804.0489 [hep-th]].

[11] G. Mason and M.P. Tuite, On genus two Riemann surfaces formed from sewn tori, Commun. Math. Phys. 270 (2007), 587 [arXiv:math/0603088].

[12] G. Mason and M.P. Tuite, Free bosonic vertex operator algebras on genus two Riemann surfaces I, arXiv:0912.0117 [math.QA].

[13] M.P. Tuite, Genus two meromorphic conformal field theories, arXiv:math/9910136.

[14] P. Di Vecchia, F. Pezzella, M. Frau, K. Hornfeck, A. Lerda and S. Sciuto, $N$ point $g$ loop vertex for a free bosonic theory with vacuum charge $Q$, Nucl. Phys. B322 (1989), 317. 
[15] P. Di Vecchia, F. Pezzella, M. Frau, K. Hornfeck, A. Lerda and S. Sciuto, $N$ point $g$ loop vertex for a free fermionic theory with arbitrary spin, Nucl. Phys. B333 (1990), 635.

[16] A. Frizzo, L. Magnea and R. Russo, Scalar field theory limits of bosonic string amplitudes, Nucl. Phys. B579 (2000), 379 [arXiv:hep-th/9912183].

[17] L. Magnea, R. Russo and S. Sciuto, Two-loop Euler-Heisenberg effective actions from charged open strings, Int. J. Mod. Phys. A21 (2006), 533 [arXiv:hep-th/0412087].

[18] R. Russo and S. Sciuto, The twisted open string partition function and Yukawa couplings, JHEP 0704 (2007), 030 [arXiv:hep-th/0701292].

[19] D. Duò, R. Russo and S. Sciuto, New twist field couplings from the partition function for multiply wrapped D-branes, JHEP 0712 (2007), 042 [arXiv:0709.1805 [hep-th]].

[20] Y. Zhu, Vertex operator algebras, elliptic functions and modular forms, Caltech preprint (1990), J. Amer. Math. Soc. 9 (1996), 237.

[21] G. Höhn, Selbstduale Vertexoperatorsuperalgebren und das Babymonster, Ph.D. thesis (Bonn 1995), Bonner Mathematische Schriften 286 (1996), [arXiv:0706.0236 [math.QA]].

[22] M. Ozeki, On basis problem for Siegel modular forms of degree 2, Acta Arith. 31(1) (1976), 17.

[23] S. Böcherer, Über die Fourier-Jacobi-Entwicklung Siegelscher Eisensteinreihen, Math. Z. 183 (1983), 21.

[24] R. Blumenhagen, M. Flohr, A. Kliem, W. Nahm, A. Recknagel and R. Varnhagen, $W$ algebras with two and three generators, Nucl. Phys. B361 (1991), 255.

[25] W. Li, W. Song and A. Strominger, Chiral gravity in three dimensions, JHEP 0804 (2008), 082 [arXiv:0801.4566 [hep-th]].

[26] D. Grumiller and N. Johansson, Instability in cosmological topologically massive gravity at the chiral point, JHEP 0807 (2008), 134 [arXiv:0805.2610 [hep-th]].

[27] M. Henneaux, C. Martinez and R. Troncoso, Asymptotically anti-de Sitter spacetimes in topologically massive gravity, Phys. Rev. D79 (2009), 081502 [arXiv:0901.2874 [hep-th]]. 
362 Matthias R. Gaberdiel, Christoph A. Keller and Roberto Volpato

[28] A. Maloney, W. Song and A. Strominger, Chiral gravity, log gravity and extremal CFT, arXiv:0903.4573 [hep-th].

[29] K. Skenderis, M. Taylor and B. C. van Rees, Topologically massive gravity and the AdS/CFT correspondence, JHEP 0909 (2009), 045 [arXiv:0906.4926 [hep-th]].

[30] D. Grumiller and I. Sachs, $A d S_{3} / L C F T_{2}$ - correlators in cosmological topologically massive gravity, arXiv:0910.5241 [hep-th].

[31] M.R. Gaberdiel, S. Gukov, C.A. Keller, G.W. Moore and H. Ooguri, Extremal $N=(2,2)$ 2d conformal field theories and constraints of modularity, Commun. Number Theory and Physics 2 (2008), 743 [arXiv:0805.4216 [hep-th]].

[32] A.N. Schellekens, Meromorphic $c=24$ conformal field theories, Commun. Math. Phys. 153 (1993), 159 [arXiv:hep-th/9205072].

[33] P. Goddard, Meromorphic conformal field theory in 'Infinite dimensional Lie algebras and Lie groups: Proceedings of the CIRM Luminy Conference, 1988', ed. Victor Kac, World Scientific, Singapore 1989, 556.

[34] E.A. Bender and E.R Canfield, The asymptotic number of labeled graphs with given degree sequences, J. Combinatorial Theory A24 (1978), 296.

[35] B. Bollobás, The asymptotic number of unlabelled regular graphs, J. London Math. Soc. 26 (1982), 201.

[36] G.-B. Chae, Asymptotic number of general cubic graphs with given connectivity, J. Korean Math. Soc. 42 (2005), 1187.

[37] G. van der Geer, Siegel modular forms and their applications, in 'The 1-2-3 of modular forms', ed. Kristian Ranestad, Universitext, Springer, Berlin, 2008, 181.

[38] J.-I. Igusa, On Siegel modular forms of genus two, Amer. J. Math. 84 (1962), 175.

[39] M. Eichler and D. Zagier, The theory of Jacobi forms, Progress in Mathematics, 55, Birkhäuser Boston Inc., Boston, MA, 1985.

[40] G.B. Segal, The definition of conformal field theory, in 'Topology, geometry and quantum field theory', London Math. Soc. Lecture Note Ser., 308, Cambridge University Press, Cambridge, 2004, p. $421 \mathrm{ff}$. 
Genus two partition functions of chiral conformal field theories 363

[41] A. McIntyre and L.A. Takhtajan, Holomorphic factorization of determinants of Laplacians on Riemann surfaces and a higher genus generalization of Kronecker's first limit formula, Geom. Funct. Anal. 16 (2006), 1291 [arXiv:math/0410294].

[42] G.W. Moore, J. Harris, P. Nelson and I. Singer, Modular forms and the cosmological constant, Phys. Lett. B178 (1986), 167 [Erratum-ibid. B 201 (1988), 579].

[43] J. Harris and I. Morrison, Moduli of curves, Graduate Texts in Mathematics, 187, Springer, New York, 1998.

[44] J. Harris and I. Morrison, Slopes of effective divisors on the moduli space of stable curves, Invent. Math. 99 (1990), 321.

[45] S.-L. Tan, On the slopes of the moduli spaces of curves, Internat. J. Math. 9(1) (1998), 119.

[46] G. Farkas and M. Popa, Effective divisors on $\overline{\mathcal{M}}_{g}$, curves on $K 3$ surfaces, and the slope conjecture, J. Algebraic Geom. 14 (2005), 241.

[47] R. Pandharipande, Descendent bounds for effective divisors on the moduli space of curves, arXiv:0805.0601 [math.AG].

Institut Für TheORETISChe PHYSIK

ETH ZÜRICH, 8093 ZÜRICH

SWITZERLAND

E-mail addresses: gaberdiel@itp.phys.ethz.ch, volpato@itp.phys.ethz.ch

California Institute of Technology

PASADENA, CA 91125

USA

E-mail address: ckeller@theory.caltech.edu

ReCeived MARCH 4, 2010 
